\title{
From black holes to quivers
}

\author{
Jan Manschot, ${ }^{a, b}$ Boris Pioline ${ }^{c, d}$ and Ashoke Sen ${ }^{e}$ \\ ${ }^{a}$ Bethe Center for Theoretical Physics, Physikalisches Institut, Universität Bonn, \\ Nussallee 12, 53115 Bonn, Germany \\ ${ }^{b}$ Max Planck Institute for Mathematics, \\ Vivatsgasse 7, 53111 Bonn, Germany \\ ${ }^{c}$ CERN PH-TH, CERN, \\ Case C01600, CH-1211 Geneva 23, Switzerland \\ ${ }^{d}$ Laboratoire de Physique Théorique et Hautes Energies, CNRS UMR 7589, \\ Université Pierre et Marie Curie, \\ 4 place Jussieu, 75252 Paris cedex 05, France \\ ${ }^{e}$ Harish-Chandra Research Institute, \\ Chhatnag Road, Jhusi, Allahabad 211019, India \\ E-mail: manschot@uni-bonn.de, pioline@lpthe.jussieu.fr, sen@hri.res.in
}

ABSTRACT: Middle cohomology states on the Higgs branch of supersymmetric quiver quantum mechanics - also known as pure Higgs states - have recently emerged as possible microscopic candidates for single-centered black hole micro-states, as they carry zero angular momentum and appear to be robust under wall-crossing. Using the connection between quiver quantum mechanics on the Coulomb branch and the quantum mechanics of multi-centered black holes, we propose a general algorithm for reconstructing the full moduli-dependent cohomology of the moduli space of an arbitrary quiver, in terms of the BPS invariants of the pure Higgs states. We analyze many examples of quivers with loops, including all cyclic Abelian quivers and several examples with two loops or non-Abelian gauge groups, and provide supporting evidence for this proposal. We also develop methods to count pure Higgs states directly.

KeYwords: Black Holes in String Theory, D-branes, Black Holes

ARXiv EPRINT: 1207.2230 


\section{Contents}

1 Introduction and summary 1

2 Poincaré polynomials from pure Higgs states $\quad 7$

2.1 Refined index from single-centered black holes $\quad 7$

2.2 A Coulomb branch formula for quiver Poincaré polynomials 10

2.3 A Coulomb branch formula for Hodge numbers 12

2.4 Quiver Poincaré polynomial from the Higgs branch analysis 13

2.5 Cohomology of quivers without superpotential and HN recursion method 17

3 Abelian three-node quiver $\quad 19$

3.1 Identifying the contributing collinear configurations 20

$\begin{array}{lll}3.2 & \text { Three-node quiver without loop } & 22\end{array}$

$\begin{array}{lll}3.3 & \text { Three node quiver with loop } & 23\end{array}$

4 Cyclic quivers $\quad 26$

4.1 Coulomb branch analysis 26

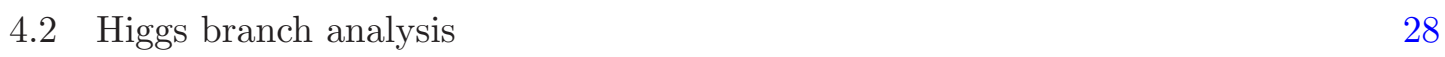

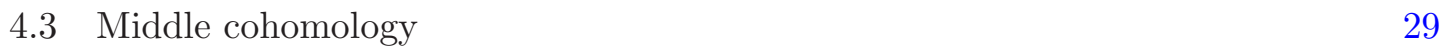

5 Quivers with two loops $\quad 32$

5.1 Abelian four-node, two-loop quivers 32

5.1.1 Example with only 3-center scaling solutions 33

5.1.2 Example with 4-center scaling solutions 36

$\begin{array}{lll}5.2 & \text { A 5-node quiver with nested scaling configurations } & 38\end{array}$

6 Non-Abelian quivers $\quad 41$

6.1 Rank $(1,1,2) \quad 41$

6.2 Rank $(1,1, N) \quad 45$

6.3 Rank $(1,2,2) \quad 48$

\section{Introduction and summary}

$\mathcal{N}=2$ supersymmetric string theories are known to possess supersymmetric configurations involving multiple black holes [1-4]. An interesting problem is to compute the spectrum of BPS states of this multi-centered configuration. A useful quantity that encodes information about not only the total number of states but also the angular momentum $J_{3}$ carried by the states is the refined 'index' $\Omega_{\text {ref }}(\gamma ; y, z) \equiv \operatorname{Tr}^{\prime}(-y)^{2 J_{3}}$. Here the trace is taken over all states carrying a given total electric and magnetic charges, collectively denoted by $\gamma$, 
after factoring out the trace over the degrees of freedom associated with the center of mass of the system. The parameter $z$ refers to the dependence on the values of the moduli at spatial infinity, which is governed by the well-known wall-crossing formulaes [5-7]. For $y=1, \Omega_{\text {ref }}(\gamma ; 1, z)$ reduces to the usual index $\operatorname{Tr}^{\prime}(-1)^{F}$, or equivalently the second helicity supertrace $[8,9] .^{1}$

Recently, drawing inspiration from various other related studies [6, 7, 11-14], we proposed a specific formula for the refined index $\Omega_{\text {ref }}(\gamma ; y, z)$ carried by a multi-centered black hole system in terms of the refined index $\Omega_{\text {ref }}^{\mathrm{S}}(\alpha ; y)$ of single centered black holes [15]. One of the virtues of this formula is that it incorporates the full dependence on the asymptotic moduli consistently with wall-crossing, leaving only moduli-independent coefficients $\Omega_{\text {ref }}^{\mathrm{S}}(\alpha ; y)$ to be determined. On the other hand, at a fixed point in moduli space, the formula does not directly give any information on $\Omega_{\text {ref }}(\gamma ; y)$, since the number of input variables - the single centered refined index $\Omega_{\text {ref }}^{\mathrm{S}}(\alpha ; y)$ for each charge vector $\alpha$ - is equal to the number of quantities to be computed - the total index $\Omega_{\text {ref }}(\gamma ; y)$ for each charge vector $\gamma$. However the formula becomes significant when combined with the observation that single centered supersymmetric black holes must carry strictly zero angular momentum [16-18] and therefore $\Omega_{\mathrm{ref}}^{\mathrm{S}}(\alpha ; y)$ must be independent of $y$. This drastically reduces the number of input parameters to a single constant for each charge vector $\alpha$, in terms of which the formula of [15] expresses the refined indices $\Omega_{\text {ref }}(\gamma ; y, z)$. This gives predictions for the $y$ dependence of the refined index of multi-centered black hole configurations in $\mathcal{N}=2$ supersymmetric string theories, which could be tested if the same index was computable by other means.

Unfortunately at present we do not have an independent way of computing the refined index of general multi-centered black hole configurations in $\mathcal{N}=2$ supersymmetric string theories. However we can construct a subset of these black hole micro-states in type II string theory compactified on Calabi-Yau spaces as bound states of elementary D-branes wrapped on various cycles of the internal space. When the central charges of the constituents nearly align, the dynamics of this system is described by an $\mathcal{N}=4$ supersymmetric quiver quantum mechanics [2] containing vector and chiral multiplets characterized by a superpotential and a set of Fayet-Iliopoulos (FI) parameters. When the FI parameters are large the vector multiplets can be integrated out and the dynamics is described by an effective theory for the chiral multiplets. The refined index $\Omega_{\text {ref }}(\gamma ; y)$ is determined by the Poincaré polynomial of the moduli space $\mathcal{M}$ of classical vacua of this effective theory, also known as the Higgs branch, which can often be computed explicitly.

On the other hand when the FI parameters are small, the dynamics of the theory can be described by an effective theory of the vector multiplets, with the chiral multiplets integrated out. This effective theory - known as the Coulomb branch theory - turns

\footnotetext{
${ }^{1}$ While the index $\operatorname{Tr}^{\prime}(-1)^{F}$ is protected and depends only on the values of the vector multiplet scalars $z$ at infinity, the refined index $\Omega_{\text {ref }}$ is not and may depend on both the vector multiplet (VM) and hypermultiplet (HM) scalars. In particular, it need not be the same at strong and weak coupling, yet it is expected that the dependence on the VM scalars obeys the motivic wall crossing formula of [5-7] in both regimes [6]. In this paper as in $[6,15]$, we work at fixed value of the HM scalars. In $\mathcal{N}=2$ gauge theories, a variant of the refined index known as the protected spin character does exist, thanks to the existence of a SU(2) R-symmetry [10]. We expect that our formulae also apply in this case with $\Omega_{\text {ref }}$ replaced by the protected spin character.
} 
out to be identical to the quantum mechanics of multi-centered black holes. Naively, if $r$ is the total rank of the gauge group of the quiver quantum mechanics, one would expect the Coulomb branch theory to describe a system of $r$ black holes (some of which could be identical), with each center carrying charge $\alpha$ associated to one of the nodes and unit degeneracy $\Omega_{\text {ref }}^{S}(\alpha)=1$. While this is indeed so for quivers without closed loops, this prescription however fails to reproduce the full spectrum computed from the Higgs branch in cases where the moduli space of the Coulomb branch has singularities associated with so called scaling solutions, where three or more centers can come arbitrarily close to each other $[4,19]$. In such cases, the general formula proposed in [15] allows to compensate for this failure by adding to the naive Coulomb branch result contributions from multiblack hole configurations with less than $r$ centers, with the new centers carrying composite charges. The contribution from these new configurations are parametrized by the single centered black hole indices $\Omega_{\text {ref }}^{\mathrm{S}}(\alpha)$ carried by the new centers. This general formula can then be compared with the Poincaré polynomial of the Higgs branch. Again, since $\Omega_{\text {ref }}(\gamma ; y)$ is not protected, there is a priori no guarantee that the Higgs and the Coulomb branch results for $\Omega_{\text {ref }}(\gamma ; y)$ should agree. Nevertheless our analysis of several examples shows that the two results do agree, indicating that this quantity is more robust than what naive arguments based on supersymmetry would suggest.

From the description given above it is clear that in order to be able to carry out the computation of $\Omega_{\mathrm{ref}}(\gamma ; y)$ on the Coulomb branch, there should exist a notion of 'single-centered micro-states', which carry zero angular momentum and whose existence is independent of the moduli at infinity, such that their refined index $\Omega_{\text {ref }}^{\mathrm{S}}(\alpha)$ be independent of both $y$ and $z$. Recent work indicates that this role may be taken by a subset of micro-states described by the middle cohomology ${ }^{2}$ of the Higgs branch $[19,20]$. Indeed, such states are invariant under the Lefschetz $\mathrm{SU}(2)$ action on the total cohomology $H^{*}(\mathcal{M}, \mathbb{Z})$, which realizes spatial rotations in real space. Moreover, these states appear to be robust under deformation of the superpotential and under wall-crossing, unlike the rest of the cohomology which jumps across walls of marginal stability. In fact, it has been observed, in the special case of the threenode quiver with a loop, that the complement of the middle cohomology is in one-to-one correspondence $^{3}$ with states on the Coulomb branch of the quiver quantum mechanics. In contrast a subset of the the middle cohomology states have no counterpart on the Coulomb side, hence deserving the name of 'pure Higgs' or 'intrinsic Higgs' states [19, 20]. The only way to incorporate these states in the Coulomb branch analysis is to add their contribution by hand as the contribution from single-centered black holes, thereby forcing us to identify the micro-states of single centered black holes with pure Higgs states. In this paper, we shall give evidence that a generalized version of these properties continues to hold in a large class of quivers, including quivers with more than one loop or non-Abelian gauge groups.

\footnotetext{
${ }^{2}$ For brevity, we use the phrase 'middle cohomology' to refer to the part of $H^{d}(\mathcal{M}, \mathbb{Z})$ which is invariant under $\mathrm{SU}(2)$ Lefschetz rotations, where $d$ is the complex dimension of $\mathcal{M}$.

${ }^{3}$ For quivers without loops, such that all charge vectors lie in a two-dimensional plane, this equivalence was proven at the level of refined indices in [21]. We believe that the assumption that all charge vectors lie in a two-dimensional plane could be relaxed.
} 
For the reader's convenience we shall now summarize our prediction for the Poincaré polynomial of quiver moduli spaces. Let us consider a quiver with $K$ nodes, carrying $\mathrm{U}\left(N_{1}\right) \times \mathrm{U}\left(N_{2}\right) \times \cdots \mathrm{U}\left(N_{K}\right)$ gauge group, and a number $\gamma_{\ell k}$ of $\left(N_{\ell}, \bar{N}_{k}\right)$ representation of $\mathrm{U}\left(N_{\ell}\right) \times \mathrm{U}\left(N_{k}\right)$. A negative $\gamma_{\ell k}$ indicates $-\gamma_{\ell k}=\gamma_{k \ell}$ number of $\left(\bar{N}_{\ell}, N_{k}\right)$ representation of $\mathrm{U}\left(N_{\ell}\right) \times \mathrm{U}\left(N_{k}\right)$. Also let $c_{\ell}$ be the Fayet-Iliopoulos (FI) parameter associated with the Abelian factor at the $\ell$-th node, subject to the condition $\sum_{\ell} N_{\ell} c_{\ell}=0$. Such a quiver is pictorially represented by $\gamma_{\ell k}$ arrows connecting the node $\ell$ to the node $k$, with the arrows being directed from $\ell$ to $k$ if $\gamma_{\ell k}$ is positive. The construction of the quiver moduli space begins by introducing a set of complex variables $\phi_{\ell k, \alpha, s s^{\prime}}$ for every pair $\ell, k$ for which $\gamma_{\ell k}>0$. Here $\alpha$ runs over $\gamma_{\ell k}$ values, $s$ is an index labelling the fundamental representation of $\mathrm{U}\left(N_{\ell}\right)$ and $s^{\prime}$ is an index representing the anti-fundamental representation of $\mathrm{U}\left(N_{k}\right)$. The moduli space of classical vacua is the space spanned by these variables $\left\{\phi_{\ell k, \alpha, s s^{\prime}}\right\}$ subject to the following D-term and F-term constraints:

$$
\begin{gathered}
\sum_{\substack{k, s, t, s^{\prime} \\
\gamma_{\ell k}>0}} \phi_{\ell k, \alpha, s s^{\prime}}^{*} T_{s t}^{a} \phi_{\ell k, \alpha, t s^{\prime}}-\sum_{\substack{k, s, t, s^{\prime} \\
\gamma_{k \ell \ell}>0}} \phi_{k \ell, \alpha, s^{\prime} s}^{*} T_{s t}^{a} \phi_{k \ell, \alpha, s^{\prime} t}=c_{\ell} \operatorname{Tr}\left(T^{a}\right) \quad \forall \ell, a, \\
\frac{\partial W}{\partial \phi_{\ell k, \alpha, s s^{\prime}}}=0 .
\end{gathered}
$$

Here $T^{a}$ 's are the generators of the $\mathrm{U}\left(N_{\ell}\right)$ gauge group, and $W$ is a gauge invariant superpotential holomorphic in the variables $\phi_{\ell k, \alpha, s s^{\prime}}$. For every closed loop in the quiver we can construct gauge invariant polynomials by taking the products of $\phi_{\ell k, \alpha, s s^{\prime}}$ along the closed loop and the superpotential $W$ is an arbitrary linear combinations of such gauge invariant polynomials. Besides the constraints given in (1.1), the variables $\left\{\phi_{\ell k, \alpha, s s^{\prime}}\right\}$ are also subject to identification under the $\prod_{\ell} \mathrm{U}\left(N_{\ell}\right)$ gauge transformations. The resulting manifold $\mathcal{M}$, which we refer to as the quiver moduli space, describes the space of classical vacua on the Higgs branch of the supersymmetric quantum mechanics. The associated refined index $\operatorname{Tr}^{\prime}(-1)^{2 J_{3}}$ is given by the Laurent polynomial

$$
Q(\mathcal{M} ; y)=(-y)^{-d} P(\mathcal{M} ;-y)=\sum_{p=1}^{2 d} b_{p}(\mathcal{M})(-y)^{p-d}
$$

where $d$ is the complex dimension of $\mathcal{M}$, the $b_{p}$ 's are its topological Betti numbers, and $P(\mathcal{M} ; t)=\sum_{p=1}^{2 d} b_{p}(\mathcal{M}) t^{p}$ is the Poincaré polynomial. The analysis of this paper gives an algorithm for computing $Q(\mathcal{M} ; y)$ as follows.

We first assign to each node $\ell$ a basis vector $\gamma_{\ell}=(0, \ldots, 1,0, \ldots)$ in an abstract vector space $\mathbb{Z}^{K}$, and introduce a symplectic inner product ${ }^{4}$

$$
\left\langle\gamma, \gamma^{\prime}\right\rangle \equiv\left(\gamma^{\prime}, \gamma\right)-\left(\gamma, \gamma^{\prime}\right), \quad\left(\gamma, \gamma^{\prime}\right) \equiv \sum_{\ell=1}^{K} n_{\ell} n_{\ell}^{\prime}-\sum_{\substack{\ell, k=1 \\ \gamma_{\ell k}>0}}^{K} n_{\ell} n_{k}^{\prime} \gamma_{\ell k}
$$

\footnotetext{
${ }^{4}$ The quadratic form $\left(\gamma, \gamma^{\prime}\right)$ and vector $\beta$ are known in the mathematical literature on quivers as the Euler form and dimension vectors. We consider the dimension vector and FI terms to be part of the data of the quiver, in deviance from mathematical practice.
} 
between the elements $\beta=\sum_{\ell=1}^{K} n_{\ell} \gamma_{\ell}$. It follows that for the basis vectors, $\left\langle\gamma_{\ell}, \gamma_{k}\right\rangle=\gamma_{\ell k}$. We denote by $\Gamma \subset \mathbb{Z}^{K}$ the collection of vectors $\beta=\sum_{\ell=1}^{K} n_{\ell} \gamma_{\ell}$ where $n_{\ell}$ are non-negative integers, and by $\mathcal{C}_{\beta}$ the hyperplane $\sum_{\ell=1}^{K} n_{\ell} c_{\ell}=0$ in the space of real vectors $c=\sum_{\ell=1}^{K} c_{\ell} \gamma_{\ell} \in$ $\mathbb{R}^{K}$. To any vectors $\beta \in \Gamma$ and $c \in \mathcal{C}_{\beta}$, we associate a quiver $\mathcal{Q}(\beta, c)$ with $K$ nodes, $\gamma_{\ell k}$ arrows connecting the node $\ell$ to the node $k$, gauge group $\mathrm{U}\left(n_{1}\right) \times \mathrm{U}\left(n_{2}\right) \times \cdots \mathrm{U}\left(n_{K}\right)$, and FI parameters $\left\{c_{1}, \cdots c_{K}\right\}$. If some of the $n_{\ell}$ 's vanish we just drop the corresponding nodes.

This construction produces a family of quivers which contains the original quiver $\mathcal{Q}(\gamma, c)$ with $\gamma=\sum_{\ell} N_{\ell} \gamma_{\ell}$ as a special case. Let $Q(\gamma ; y)$ be the corresponding Laurent polynomial introduced in (1.2). Our conjectured formula for $Q(\gamma ; y)$, which we shall often refer to as the Coulomb branch formula, takes the form:

$$
\begin{aligned}
Q(\gamma ; y)= & \sum_{m \mid \gamma} \mu(m) m^{-1} \frac{y-y^{-1}}{y^{m}-y^{-m}} \bar{Q}\left(\gamma / m ; y^{m}\right) \\
\bar{Q}(\gamma ; y)= & \sum_{n \geq 1} \sum_{\left\{\alpha_{i} \in \Gamma\right\}, \sum_{i=1}^{n} \alpha_{i}=\gamma} \frac{1}{\operatorname{Aut}\left(\left\{\alpha_{1}, \alpha_{2}, \cdots, \alpha_{n}\right\}\right)} g_{\mathrm{ref}}\left(\alpha_{1}, \alpha_{2}, \cdots, \alpha_{n} ; y\right) \\
& \times \prod_{i=1}^{n}\left\{\sum_{\substack{m_{i} \in \mathbb{Z} \\
m_{i} \mid \alpha_{i}}} \frac{1}{m_{i}} \frac{y-y^{-1}}{y^{m_{i}}-y^{-m_{i}}}\left(\Omega_{\mathrm{ref}}^{S}\left(\alpha_{i} / m_{i} ; y^{m_{i}}\right)+\Omega_{\mathrm{scaling}}\left(\alpha_{i} / m_{i} ; y^{m_{i}}\right)\right)\right\},
\end{aligned}
$$

where $\mu(m)$ is the Möbius function, $\operatorname{Aut}\left(\left\{\alpha_{1}, \cdots \alpha_{n}\right\}\right)$ is given by $\prod_{k} s_{k}$ ! if among the set $\left\{\alpha_{i}\right\}$ there are $s_{1}$ identical vectors $\tilde{\alpha}_{1}, s_{2}$ identical vectors $\tilde{\alpha}_{2}$ etc., and $m \mid \alpha$ means that $m$ is a common divisor of $\left(n_{1}, \cdots, n_{K}\right)$ if $\alpha=\sum_{\ell} n_{\ell} \gamma_{\ell}$. The factor $g_{\mathrm{ref}}\left(\alpha_{1}, \alpha_{2}, \cdots, \alpha_{n} ; y\right)$, which we shall call the 'Coulomb index', is (the bulk contribution to) the refined index of the quantum mechanics of $n$ charged particles. It is equal to 1 for $n=1$ and

$$
\begin{aligned}
g_{\mathrm{ref}}\left(\alpha_{1}, \ldots, \alpha_{n} ; y\right) & =(-1)^{\sum_{i<j} \alpha_{i j}+n-1}\left[\left(y-y^{-1}\right)^{1-n} \sum_{p} s(p) y^{\sum_{i<j} \alpha_{i j} \operatorname{sign}\left[x_{j}-x_{i}\right]}\right] \text { for } n \geq 2 \\
\alpha_{i j} & \equiv\left\langle\alpha_{i}, \alpha_{j}\right\rangle .
\end{aligned}
$$

Here the sum over $p$ runs over all solutions to the system of $n-1$ independent algebraic equations in $n-1$ unknowns $x_{2}, \ldots x_{n}$,

$$
\sum_{\substack{j=1 \\ j \neq i}}^{n} \frac{\alpha_{i j}}{\left|x_{i}-x_{j}\right|}=\hat{c}_{i}, \quad x_{i} \in \mathbb{R}, \quad \text { for } 1 \leq i \leq n,
$$

with $x_{1}$ fixed to any value, and $s(p)= \pm 1$ is a certain sign given in (2.3). The prescriptions for enumerating the solutions to (1.6) are detailed in section 2. The coefficients $\hat{c}_{i}$ are determined in terms of the FI parameters $c_{i}$ by $\hat{c}_{i}=\sum_{\ell} A_{i \ell} c_{\ell}$ whenever $\alpha_{i}=\sum_{\ell} A_{i \ell} \gamma_{\ell}$. From the restriction $\sum_{i} \alpha_{i}=\gamma$ and that $\sum_{\ell} N_{\ell} c_{\ell}=0$ it follows that $\sum_{i} \hat{c}_{i}=0$, as required for the consistency of the equations (1.6).

The symbols $\Omega_{\text {ref }}^{\mathrm{S}}$ 's appearing in (1.4) represent the refined indices of single centered micro-states and are given as follows. First of all we have $\Omega_{\text {ref }}^{\mathrm{S}}\left(\gamma_{\ell} ; y\right)=1$ for $1 \leq \ell \leq K$. For any other $\beta \in \Gamma, \Omega_{\text {ref }}^{\mathrm{S}}(\beta ; y)$ is an unknown $y$-independent constant. For reason that 
will become clear soon we shall refrain from setting $\Omega_{\text {ref }}^{\mathrm{S}}(\beta ; y)=\Omega_{\text {ref }}^{\mathrm{S}}(\beta)$ until we determine

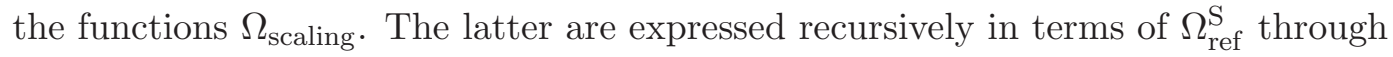

$$
\Omega_{\text {scaling }}(\alpha ; y)=\sum_{\substack{\left\{\beta_{i} \in \Gamma\right\},\left\{m_{i} \in \mathbb{Z}\right\} \\ m_{i} \geq 1, \sum_{i} m_{i} \beta_{i}=\alpha}} H\left(\left\{\beta_{i}\right\} ;\left\{m_{i}\right\} ; y\right) \prod_{i} \Omega_{\mathrm{ref}}^{S}\left(\beta_{i} ; y^{m_{i}}\right),
$$

where the functions $H\left(\left\{\beta_{i}\right\} ;\left\{k_{i}\right\} ; y\right)$ are determined as follows. Firstly, when the number of $\beta_{i}$ 's is less that three, $H\left(\left\{\beta_{i}\right\} ;\left\{k_{i}\right\} ; y\right)$ vanishes. For three or more number of $\beta_{i}$ 's, we note that the expression for $Q\left(\sum_{i} k_{i} \beta_{i} ; y\right)$ given in (1.4) contains a term proportional to $H\left(\left\{\beta_{i}\right\} ;\left\{k_{i}\right\} ; y\right) \prod_{i} \Omega_{\text {ref }}^{\mathrm{S}}\left(\beta_{i} ; y^{k_{i}}\right)$ arising from the choice $m=1$ in the first equation in (1.4), $n=1, \alpha_{1}=\sum_{i} k_{i} \beta_{i}, m_{1}=1$ in the second equation in (1.4), and $m_{i}=k_{i}$ in the expression for $\Omega_{\text {scaling }}\left(\sum_{i} k_{i} \beta_{i} ; y\right)$ in eq.(1.7). We fix $H\left(\left\{\beta_{i}\right\} ;\left\{k_{i}\right\} ; y\right)$ by demanding that the net coefficient of the $\prod_{i} \Omega_{\mathrm{ref}}^{\mathrm{S}}\left(\beta_{i} ; y^{k_{i}}\right)$ in the expression for $Q\left(\sum_{i} k_{i} \beta_{i} ; y\right)$ is a Laurent polynomial in $y$. This of course leaves open the possibility of adding to $H$ a Laurent polynomial. This is resolved by using the minimal modification hypothesis, which requires that $H$ must be symmetric under $y \rightarrow y^{-1}$ and vanish as $y \rightarrow \infty[15]$. We determine $H\left(\left\{\beta_{i}\right\} ;\left\{m_{i}\right\} ; y\right)$ iteratively by beginning with the $H$ 's with three $\beta_{i}$ 's and then determining successively the $H$ 's with more $\beta_{i}$ 's. $\Omega^{\mathrm{S}}$ and $H$ are expected to be independent of the FI parameters and hence can be calculated for any value of these parameters. From the algorithm for determining $H$ described above it is clear that one should retain the $y$-dependence of $\Omega_{\text {ref }}^{\mathrm{S}}$ at intermediate stages to distinguish between $\Omega_{\text {ref }}^{\mathrm{S}}\left(\beta ; y^{m}\right)$ for different values of $m$. For Abelian quivers this is not important since only $\Omega_{\text {ref }}^{\mathrm{S}}(\beta ; y)$ appear in the final expression and hence in this case we can set $\Omega_{\text {ref }}^{\mathrm{S}}(\beta ; y)=\Omega_{\text {ref }}^{\mathrm{S}}(\beta)$ from the outset.

The Coulomb branch formula (1.4)-(1.7) gives an explicit algorithm for computing the Poincaré polynomial of the quivers $\mathcal{Q}(\beta)$ for all dimension vectors $\beta \in \Gamma$ in terms of the constants $\Omega_{\mathrm{ref}}^{\mathrm{S}}(\beta)$. There is one such undetermined constant for each $\beta \in \Gamma$, representing the number of 'pure Higgs states' which cannot be determined by our algorithm and must be computed by other methods. ${ }^{5}$ It is also worth stressing that the Coulomb branch formula automatically satisfies the Kontsevich-Soibelman wall crossing formula [5]. This follows from the result of [15] that the formula for the index given there satisfies the wall crossing formula of [6], and the result of [21] showing the equivalence of the wall crossing formulæ of [5] and [6].

As is clear from the above, the main assumption in our algorithm is that there exists a class of 'single-centered black hole micro-states' which have the property that their index $\Omega_{\text {ref }}^{\mathrm{S}}(\beta)$ is independent of $y$ and robust under wall-crossing. For single centered black holes the $y$-independence of the index follows from the fact that the black hole carries zero angular momentum. This is in turn a consequence of spherical symmetry of a supersymmetric black hole together with the fact that an extremal black hole represents a collection of states in the microcanonical ensemble where all charges and angular momenta are fixed [16]. For the quiver the role of single centered black hole states is played by 'pure Higgs states' — states which are visible on the Higgs branch but not on the Coulomb branch of the supersymmetric

\footnotetext{
${ }^{5}$ If the quiver $\mathcal{Q}(\beta)$ has no oriented closed loop then $\Omega_{\text {ref }}^{\mathrm{S}}(\beta)$ as well as $\Omega_{\text {scaling }}(\beta ; y)$ are expected to vanish [4].
} 
quantum mechanics $[4,19,20]$. Since the quiver description is valid in a different region in the space of coupling constants, and since the $y$ dependence of the index is not guaranteed to be protected under a change of coupling, one might expect that for the quiver $\Omega^{\mathrm{S}}$ may be $y$-dependent. Nevertheless the recent studies in $[19,20]$ indicate that even for the quiver the $y$ independence of $\Omega^{\mathrm{S}}$ holds, which we therefore take as our working hypothesis. Needless to say, we find that this hypothesis holds in all the examples that we have analyzed.

The remainder of this work is organized as follows. In section 2 we review the general formula of [15] expressing the refined index of a multi-centered black hole system in terms of the index carried by single centered black holes. We then show how a microscopic version of this formula can be used to compute the Betti numbers of quiver moduli spaces. We also suggest an extension of this formula for computing the Hodge numbers. We then review other methods for computing the cohomology of the Higgs branch directly, using the Lefschetz hyperplane theorem, Riemann-Roch theorem and Harder-Narasimhan recursion method. In sections $3,4,5$ and 6 we apply our general methods to compute the cohomology of different kinds of quivers, and compare the results with those obtained by other methods. Section 3 deals with quivers with 3-nodes, section 4 with cyclic quivers and section 5 with more complicated quivers in which the arrows form more that one closed loop. In each case however we consider only U(1) gauge groups at the nodes. In section 6 we consider example of quivers with non-abelian gauge groups.

As this manuscript was being prepared for publication, the preprint [22] appeared on arXiv, which overlaps substantially with our results for cyclic quivers in section 4 .

\section{Poincaré polynomials from pure Higgs states}

In this section we first review the formula of [15] expressing the refined index of a general multi-centered black hole configuration in terms of the index carried by the individual centers. We shall then argue that a microscopic version of this formula can be used to constrain the form of the Poincaré and Dolbeault polynomials of quiver moduli spaces. We then describe several mathematical methods for computing the cohomology of the Higgs branch directly.

\subsection{Refined index from single-centered black holes}

Let us consider a general multi-centered black hole configuration with individual centers carrying charges $\alpha_{1}, \cdots \alpha_{n}$. The collinear equilibrium configurations of $n$ single centered black holes carrying charges $\alpha_{1}, \cdots \alpha_{n}$ in some lattice $\Gamma$ are given by the extrema of the Coulomb potential

$$
\hat{W}\left(\left\{x_{i}\right\}\right)=-\sum_{1 \leq i<j \leq n} \alpha_{i j} \operatorname{sign}\left(x_{j}-x_{i}\right) \ln \left|x_{j}-x_{i}\right|-\sum_{i=1}^{n} \hat{c}_{i} x_{i}, \quad x_{i} \in \mathbb{R},
$$

with respect to $x_{2}, \cdots x_{n}$ at fixed $x_{1}$. Here $\alpha_{i j}=\left\langle\alpha_{i}, \alpha_{j}\right\rangle$ denotes the DiracSchwinger=Zwanziger symplectic product between $\alpha_{i}$ and $\alpha_{j}$. The constants $\hat{c}_{i}$ depend on the asymptotic moduli and the charges, and satisfy $\sum_{i} \hat{c}_{i}=0$. Extremizing $W$ 
gives a system of $n-1$ equations $^{6}$

$$
\sum_{j \neq i} \frac{\alpha_{i j}}{\left|x_{i}-x_{j}\right|}=\hat{c}_{i} \quad i=2 \ldots n
$$

which are algebraic in the variables $x_{i}$ for any fixed ordering along the real axis. We let

$$
s(p)=\operatorname{sign} \operatorname{det} M,
$$

where $M_{\ell k}=\partial^{2} \hat{W} / \partial x_{\ell} \partial x_{k}$ for $2 \leq \ell, k \leq K$ is the Hessian of $\hat{W}$ with respect to the variables $x_{2}, \cdots x_{n}$ at fixed $x_{1}$. Under reflection along the $x$-axis, one has $x_{i} \mapsto-x_{i}, s(p) \mapsto(-1)^{n-1} s(p)$.

We define the Coulomb index associated to the unordered set of charges $\left\{\alpha_{i}\right\}_{i=1 \ldots n}$ by $g_{\text {ref }}\left(\alpha_{1} ; y\right)=1$ for $n=1$, and

$$
g_{\mathrm{ref}}\left(\alpha_{1}, \ldots, \alpha_{n} ; y\right)=(-1)^{\sum_{i<j} \alpha_{i j}+n-1}\left[\left(y-y^{-1}\right)^{1-n} \sum_{p} s(p) y^{\sum_{i<j} \alpha_{i j} \operatorname{sign}\left[x_{j}-x_{i}\right]}\right]
$$

for $n \geq 2$. Here the sum over $p$ runs over all solutions to (2.2), with the following understanding: If two or more centers carry mutually local charges (i.e. $\alpha_{i j}=0$ ), then the prescription of [15] is to analytically continue the charges away from their original values so that they are slightly different from each other, find the set of extrema of $W$ and then continue the charges back to their original values. ${ }^{7}$ If all centers corresponding to mutually local $\alpha_{i}$ 's in the original solution are separated then the analytic continuation has no effect. However if $M$ centers with mutually local charges coincide in the original solution, then the analytic continuation will separate their locations and pick one out of the $M$ ! possible orderings of these centers along the $x$-axis. Thus, instead of analytically continuing the charges we may adopt the equivalent prescription of counting a solution with $M$ coincident mutually local charges only once, rather than $M$ ! times.

In addition, we exclude solutions in which several centers carrying mutually non-local charges (i.e. $\alpha_{i j} \neq 0$ ) would coincide. Such singular solutions arise for certain choices of charges which allow for 'scaling solutions', where a subset (or all) of the charges can approach each other at arbitrarily small distances. In the absence of such scaling solutions, $g_{\text {ref }}\left(\alpha_{1}, \ldots, \alpha_{n} ; y\right)$ computes the refined index $\operatorname{Tr}(-y)^{2 J_{3}}$ of the quantum mechanics of $n$ charged particles interacting by Coulomb, Lorentz, Newton and scalar exchange forces. Here $y$ is a parameter conjugate to the angular momentum along the $z$ axis, and the trace is taken after factoring out the center of mass modes. Equivalently, $g_{\mathrm{ref}}\left(\alpha_{1}, \ldots, \alpha_{n} ; y\right)$ computes the index of the equivariant Dirac operator on the space of solutions to (2.2), - a compact symplectic space equipped with an Hamiltonian action of $\mathrm{SO}(3)$. The

\footnotetext{
${ }^{6}$ For multi-centered black holes, the regularity of the metric and absence of time-like curves puts additional conditions on the solutions to (2.2). We assume that no such restriction arises in the case of quivers, since all the charge vectors lie in the convex cone $N_{\ell} \geq 0$.

${ }^{7}$ If $\sum_{p} s(p) y^{\sum_{i<j} \alpha_{i j} \operatorname{sign}\left[x_{j}-x_{i}\right]}$ contains $y$-independent constant terms then the coefficient of the constant term can some time depend on the details of how we take the limit to the original values. However this does not affect the final result since the functions $H$ introduced in (2.7) precisely compensates for this.
} 
result (2.4) then arises by localizing with respect to the action $J_{3}$, whose only fixed points are the isolated collinear configurations above [6].

In the presence of scaling solutions, the space of solutions to (2.2) is non-compact, and there are additional non-isolated fixed points which can contribute to the equivariant index. We shall continue to define $g_{\text {ref }}$ as the contribution of the collinear configurations (2.4), excluding the singular scaling solutions with mutually non-local coincident centers. As we shall review, the additional contributions from the scaling solutions can be determined by the 'minimal modification hypothesis' of [15].

For $n=2$, none of these issues arises, and $g_{\text {ref }}\left(\alpha_{1}, \alpha_{2} ; y\right)$ reduces to the character of a spin $J=\frac{1}{2}\left(\left\langle\alpha_{1}, \alpha_{2}\right\rangle-1\right)$ representation [6],

$g_{\mathrm{ref}}\left(\alpha_{1}, \alpha_{2} ; y\right)=\left\{\begin{array}{l}(-1)^{\left\langle\alpha_{1}, \alpha_{2}\right\rangle+1}\left(y^{\left\langle\alpha_{1}, \alpha_{2}\right\rangle}-y^{-\left\langle\alpha_{1}, \alpha_{2}\right\rangle}\right) /\left(y-y^{-1}\right) \text { for } \operatorname{sign}\left(\left\langle\alpha_{1}, \alpha_{2}\right\rangle\right)=\operatorname{sign}\left(c_{1}\right) \\ 0 \text { for } \operatorname{sign}\left(\left\langle\alpha_{1}, \alpha_{2}\right\rangle\right)=-\operatorname{sign}\left(c_{1}\right) .\end{array}\right.$

For a general charge vector $\gamma \in \Gamma$, the refined index $\Omega_{\text {ref }}(\gamma ; y)=\operatorname{Tr}^{\prime}(-y)^{2 J_{3}}$ - where the trace is now taken over all states carrying total charge $\gamma$ after factoring out the center of mass degrees of freedom - is expressed in terms of the Coulomb indices $g_{\text {ref }}$ via $[15]^{8}$

$$
\begin{aligned}
\Omega_{\mathrm{ref}}(\gamma ; y)= & \sum_{m \mid \gamma} \mu(m) m^{-1} \frac{y-y^{-1}}{y^{m}-y^{-m}} \bar{\Omega}_{\mathrm{ref}}\left(\gamma / m ; y^{m}\right) \\
\bar{\Omega}_{\mathrm{ref}}(\gamma ; y)= & \sum_{n \geq 1} \sum_{\left\{\alpha_{i} \in \Gamma\right\}, \sum_{i=1}^{n} \alpha_{i}=\gamma} \frac{1}{\operatorname{Aut}\left(\left\{\alpha_{1}, \alpha_{2}, \cdots, \alpha_{n}\right\}\right)} g_{\mathrm{ref}}\left(\alpha_{1}, \alpha_{2}, \cdots, \alpha_{n} ; y\right) \\
& \times \prod_{i=1}^{n}\left\{\sum_{\substack{m_{i} \in \mathbb{Z} \\
m_{i} \mid \alpha_{i}}} \frac{1}{m_{i}} \frac{y-y^{-1}}{y^{m_{i}}-y^{-m_{i}}}\left(\Omega_{\mathrm{ref}}^{S}\left(\alpha_{i} / m_{i} ; y^{m_{i}}\right)+\Omega_{\mathrm{scaling}}\left(\alpha_{i} / m_{i} ; y^{m_{i}}\right)\right)\right\},
\end{aligned}
$$

where $\mu(m)$ is the Möbius function, $\Gamma$ is the charge lattice, $\Omega_{\text {ref }}^{S}(\beta ; y)$ denotes the refined index carried by a single centered black hole of charge $\beta$, and

$$
\Omega_{\text {scaling }}(\alpha ; y)=\sum_{\substack{\left\{\beta_{i} \in \Gamma\right\},\left\{m_{i} \in \mathbb{Z}\right\} \\ m_{i} \geq 1, \sum_{i} m_{i} \beta_{i}=\alpha}} H\left(\left\{\beta_{i}\right\} ;\left\{m_{i}\right\} ; y\right) \prod_{i} \Omega_{\text {ref }}^{S}\left(\beta_{i} ; y^{m_{i}}\right),
$$

for some function $H\left(\left\{\beta_{i}\right\} ;\left\{m_{i}\right\} ; y\right)$. We determine $H\left(\left\{\beta_{i}\right\} ;\left\{m_{i}\right\} ; y\right)$ by requiring that the coefficient of the $\prod_{i} \Omega_{\text {ref }}^{\mathrm{S}}\left(\beta_{i} ; y^{m_{i}}\right)$ in the expression for $\Omega_{\mathrm{ref}}\left(\sum_{i} m_{i} \beta_{i} ; y\right)$ is a Laurent polynomial in $y$. The ambiguity of adding to $H$ a Laurent polynomial is resolved by using the 'minimal modification hypothesis', which requires that $H$ must be symmetric under $y \rightarrow y^{-1}$ and vanish as $y \rightarrow \infty$ (and hence also as $y \rightarrow 0$ ). Alternatively, one could absorb $\Omega_{\text {ref }}^{S}\left(\alpha_{i} ; y\right)$ into $\Omega_{\text {scaling }}\left(\alpha_{i} ; y\right)$ at the cost of allowing $H$ to take a finite value as $y \rightarrow \infty$.

Concretely, suppose that the net coefficient of the monomial $\prod_{i} \Omega_{\mathrm{ref}}^{\mathrm{S}}\left(\beta_{i} ; y^{k_{i}}\right)$ in the expression for $\Omega_{\text {ref }}\left(\sum_{i} k_{i} \beta_{i} ; y\right)$ is given by $f(y)+H\left(\left\{\beta_{i}\right\} ;\left\{k_{i}\right\} ; y\right)$ where $f(y)$ is a known

\footnotetext{
${ }^{8}$ In [15] the formula for $\Omega_{\text {ref }}(\gamma ; y)$ was given for the case when the total charge $\gamma$ is primitive. Otherwise the same formula applies to the 'rational refined index' $\bar{\Omega}_{\mathrm{ref}}(\gamma ; y)=\sum_{m \mid \gamma} \frac{1}{m} \frac{y-y^{-1}}{y^{m}-y^{-m}} \Omega_{\mathrm{ref}}\left(\gamma / m ; y^{m}\right)$. From this we can arrive at the expression for $\Omega_{\text {ref }}(\gamma ; y)$ using the inverse transformation $\Omega_{\text {ref }}(\gamma ; y)=$ $\sum_{m \mid \gamma} \frac{\mu(m)}{m} \frac{y-y^{-1}}{y^{m}-y^{-m}} \bar{\Omega}_{\mathrm{ref}}\left(\gamma / m ; y^{m}\right)$.
} 
function, with Laurent series expansion $\sum_{n<N} f_{n} y^{-n}$ around $y=0$. It is easy to check that

$$
H\left(\left\{\beta_{i}\right\} ;\left\{k_{i}\right\} ; y\right)=f_{0}+\sum_{n \geq 1} f_{n}\left(y^{-n}+y^{n}\right)-f(y)
$$

is the unique solution to the conditions stated above. This may be rewritten as a contour integral around $u=0$,

$$
H\left(\left\{\beta_{i}\right\} ;\left\{k_{i}\right\} ; y\right)=\oint \frac{\mathrm{d} u}{2 \pi \mathrm{i}} \frac{(1 / u-u) f(u)}{(1-u y)(1-u / y)}-f(y) .
$$

We determine $H\left(\left\{\beta_{i}\right\} ;\left\{m_{i}\right\} ; y\right)$ iteratively by beginning with the $H$ 's with least possible number of $\beta_{i}$ 's (three) and then determining successively the $H$ 's with larger number of $\beta_{i}$ 's. Physically $\Omega_{\text {scaling }}$ and hence $H$ represent the correction to the index due to the presence of scaling solutions [4].

In the formulae $(2.6),(2.7)$ the $\Omega_{\text {ref }}^{\mathrm{S}}(\gamma ; y)$, representing the index carried by the single centered black holes, must be independent of $y$ since single centered BPS black holes carry zero angular momentum [16]. Furthermore $H$ and $\Omega^{\mathrm{S}}$ are expected to be independent of the values of the parameters $\hat{c}_{i}$, as the jumps of the refined index across walls of marginal stability in the space of the parameters $\hat{c}_{i}$ is already captured by the Coulomb indices $g_{\text {ref }}$ [15].

\subsection{A Coulomb branch formula for quiver Poincaré polynomials}

In the weak coupling limit, the dynamics of multi-centered black holes is described by a quantum mechanics with $\mathcal{N}=4$ supersymmetry, whose matter content is captured by a certain quiver [2]. For $N_{\ell}$ centers of charge $\gamma_{\ell}$ for $\ell=1, \cdots K$, the corresponding quiver has $K$ nodes labelled by the integer $\ell$, with a complex vector space of dimension $N_{\ell}$ attached to the node $\ell$ and $\left|\gamma_{\ell k}\right|$ arrows connecting the node $\ell$ to the node $k$ if $\gamma_{\ell k}>0$, or connecting the node $k$ to the node $\ell$ if $\gamma_{\ell k}<0$. The nodes represent $\mathrm{U}\left(N_{\ell}\right)$ vector multiplets, while the arrows represent $\left|\gamma_{\ell k}\right|$ chiral multiplets $\phi_{\ell k, \alpha, s s^{\prime}}$ in the bifundamental of $\mathrm{U}\left(N_{\ell}\right) \times \mathrm{U}\left(N_{k}\right)$ (or its complex conjugate, if $\gamma_{\ell k}<0$ ). In addition, to each node we associate a constant $c_{\ell}$ labelling the coefficient of the FI parameter for the $\mathrm{U}(1)$ factor in $\mathrm{U}\left(N_{\ell}\right)$, such that $\sum_{\ell} N_{\ell} c_{\ell}=0$. Finally, the superpotential $W$ for the chiral multiplets is a generic sum of gauge-invariant monomials associated to each oriented loop in the quiver.

At low energies, the supersymmetric quantum mechanics admits two different effective descriptions, the Coulomb branch description, which is valid in the region where the vevs of the vector multiplet scalars are large, so that the chiral multiplets can be integrated out, and the Higgs branch description, valid in the region where the vevs of the chiral multiplets are large, so that the vector multiplets can be integrated out. In this subsection we shall focus on the Coulomb branch description. The flat directions of the potential on the Coulomb branch turn out to reproduce the moduli space of supersymmetric configurations of multicentered BPS black holes in $\mathcal{N}=2$ supergravity, with the FI parameters determined by the values of the scalar fields at spatial infinity [2]. This allows us to borrow the results on multi-centered black hole quantum mechanics, reviewed in the previous section, to analyze the refined index of the quiver quantum mechanics on the Coulomb branch. Using (1.2), we can then use this result to make predictions for the Poincaré polynomial of the moduli 
space of quivers on the Higgs branch. This can then be compared with a direct computation of the same Poincaré polynomial using methods to be discussed in sections 2.4-2.5.

We denote by $Q(\gamma ; y)$ the refined index of the supersymmetric quantum mechanics associated with the quiver $\mathcal{Q}(\gamma, c)$ for $\gamma=\sum_{\ell} N_{\ell} \gamma_{\ell}$. The refined index $Q(\gamma ; y)$ can be computed using the results of section 2.1 with the following understanding:

1. The role of the charge lattice is now played by the set of charges of the form $\sum_{\ell} m_{\ell} \gamma_{\ell}$ with $m_{\ell} \in \mathbb{Z}$. Furthermore only charge vectors with non-negative $m_{\ell}$ can appear in the sums over charge vectors in (2.6), (2.7).

2. The index $\Omega_{\mathrm{ref}}\left(\sum_{\ell} N_{\ell} \gamma_{\ell} ; y\right)$ appearing on the 1.h.s. of $(2.6)$ is interpreted as the Laurent polynomial (2.18) of the Higgs branch of the quiver with gauge group $\mathrm{U}\left(N_{\ell}\right)$ at the $\ell$ th node.

3. In evaluating the 'Coulomb index' $g_{\text {ref }}\left(\alpha_{1}, \cdots \alpha_{n} ; y\right)$ appearing in (2.6), the parameters $\hat{c}_{i}$ are determined in terms of the FI parameters through $\hat{c}_{i}=\sum_{\ell} A_{i \ell} c_{\ell}$ whenever $\alpha_{i}=\sum_{\ell} A_{i \ell} \gamma_{\ell}$. Since only combinations such that $\sum_{i} \alpha_{i}=\gamma=\sum_{\ell} N_{\ell} \gamma_{\ell}$ appear, the condition $\sum_{i} \hat{c}_{i}=\sum_{\ell} N_{\ell} c_{\ell}=0$ is automatically satisfied.

4. The quantities $\Omega_{\mathrm{ref}}^{\mathrm{S}}(\beta ; y)$ appearing on the r.h.s. of $(2.6)$ and $(2.7)$ are interpreted as the number of 'single centered black hole micro-states' with charge $\beta$. These are the 'pure Higgs' or 'intrinsic Higgs' states which originate from the middle cohomology of the quiver or one of its subquivers. They are assumed to be $y$-independent, although it is useful to retain the dependence on $y$ to carry out the algorithm explained in section 2.1 .

5. For any of the basis vectors $\gamma_{\ell}$, we set $\Omega_{\text {ref }}^{\mathrm{S}}\left(N \gamma_{\ell} ; y\right)=1$ for $N=1$ and zero if $N>1$, since each node of the quiver is assumed to represent a single state of zero angular momentum (using the formulas in 2.5, one finds that also mathematically the generalized DT-invariant vanishes for quiver representations with dimension $N$ at the node $\ell$ and dimension 0 at the other nodes).

It is important to note that even though the quiver quantum mechanics maps to the quantum mechanics describing the dynamics of multiple black holes, for an actual system of black holes the quiver quantum mechanics counts only part of the black hole micro-states. In particular if we associate to each node of the quiver an elementary constituent carrying charge $\gamma_{\ell}$, then the quiver quantum mechanics counts only a subset of the micro-states which carry a total charge $\gamma=\sum_{\ell} N_{\ell} \gamma_{\ell}$, - namely those which can be built from elementary constituents carrying charges $\gamma_{1}, \cdots, \gamma_{K}$. Other black hole micro-states with the same total charge could arise from bound states of other elementary constituents described by different types of quivers. Similarly $\Omega_{\mathrm{ref}}^{\mathrm{S}}(\beta ; y)$ need not count all states of a single centered black hole of charge $\beta$, but counts only those states which can be built from the elementary constituents carrying charges $\gamma_{1}, \cdots \gamma_{K}$. For the quiver, $\Omega_{\mathrm{ref}}^{\mathrm{S}}(\beta ; y)$ represent contribution from states which are elementary from the point of view of the Coulomb branch but are composite from the point of view of the Higgs branch except when $\beta$ coincides with one of the basis vectors $\gamma_{\ell}$. 
This microscopic re-interpretation of the formulae (2.6), (2.7) leads to the algorithm for computing $Q(\mathcal{M} ; y)$ summarized in section 1 . Like its macroscopic counterpart [15], this procedure leaves the 'single-centered micro-state degeneracies' $\Omega_{\text {ref }}^{\mathrm{S}}(\beta)$ for $\beta=\sum_{\ell} m_{\ell} \gamma_{\ell}$ undetermined. These can be fixed by independently computing the Euler character of the corresponding quiver moduli space, using techniques discussed in section 2.4 and section 2.5, and comparing with the prediction of (2.6) at $y=1$, where the Poincaré polynomial reduces to the Euler character. Alternatively it can be determined in terms of the middle cohomology of the quiver with $\mathrm{U}\left(m_{\ell}\right)$ gauge group at the $\ell$-th node by comparing the $y$-independent terms on both sides of (2.6) for $\gamma=\sum_{\ell} m_{\ell} \gamma_{\ell}$. Once the coefficients $\Omega_{\text {ref }}^{\mathrm{S}}(\gamma)$ have been determined, we can use (2.6) to determine the Poincaré polynomial of a quiver with arbitrary gauge groups $\prod_{\ell} \mathrm{U}\left(N_{\ell}\right)$.

\subsection{A Coulomb branch formula for Hodge numbers}

So far we have focused on the Poincaré polynomial of the quiver moduli space $\mathcal{M}$, i.e. on the topological Betti numbers $b_{i}(\mathcal{M})$. However, since $\mathcal{M}$ is a complex Kähler manifold its cohomology admits a Dolbeault decomposition

$$
H^{*}(\mathcal{M}, \mathbb{Z})=\sum_{p, q=0}^{d} H^{p, q}(\mathcal{M}, \mathbb{Z}),
$$

We shall now give an algorithm for computing the Hodge numbers $h_{p, q}(\mathcal{M})=$ $\operatorname{dim} H^{p, q}(\mathcal{M}, \mathbb{Z})$, generalizing the prescription in the previous subsection.

Let us define the Dolbeault polynomial as the Laurent polynomial in two variables

$$
\widetilde{Q}(\mathcal{M} ; y, t)=\sum_{p, q} h_{p, q}(\mathcal{M})(-y)^{p+q-d} t^{p-q} .
$$

Using the standard symmetries of the Hodge numbers $h_{p, q}=h_{d-p, d-q}=h_{q, p}$, one has

$$
\widetilde{Q}(\mathcal{M} ; y, t)=\widetilde{Q}(\mathcal{M} ; 1 / y, 1 / t)=\widetilde{Q}(\mathcal{M} ; y, 1 / t) .
$$

For $t=1, \widetilde{Q}(\mathcal{M} ; y, t)$ reduces to the Laurent polynomial $Q(\mathcal{M} ; y)$ introduced in (1.2), while for $t=1 / y$, it reduces to

$$
\widetilde{Q}(\mathcal{M} ; y, 1 / y)=(-y)^{-d} \chi\left(\mathcal{M} ; y^{2}\right)
$$

where $\chi(\mathcal{M} ; v)$ is the Hirzebruch polynomial [23]

$$
\chi(\mathcal{M} ; v)=\sum_{p, q}(-1)^{p+q} v^{q} h_{p, q}(\mathcal{M})=v^{d} \chi(\mathcal{M} ; 1 / v) .
$$

For $y=t=1, \widetilde{Q}(\mathcal{M} ; y, t)$ reduces to $(-1)^{d}$ times the Euler number $\chi(\mathcal{M})$. Finally $\widetilde{Q}(\mathcal{M} ; y, t)$ is related to the Hodge polynomial $H(u, v) \equiv h_{p, q} u^{p} v^{q}$ via $\widetilde{Q}(\mathcal{M} ; y, t)=$ $(-y)^{-d} H(-y t,-y / t)$.

The parameter $t$ is a chemical potential conjugate to the quantum number $I_{3} \equiv p-q$, which can be viewed as the remnant of a $\mathrm{SU}(2)_{R}$ symmetry in the full quiver quantum 
mechanics, before integrating out the vector multiplets to reach the Higgs branch description. For $t=y$ (or $t=1 / y$ ), the Dolbeault polynomial $\widetilde{Q}(\mathcal{M} ; y, t)$ at $t=y$ is therefore identified with the protected spin character $\operatorname{Tr}^{\prime}(-1)^{2 J_{3}} y^{2\left(I_{3}+J_{3}\right)}[10]$. On the other hand, there is much evidence ${ }^{9}$ that all states associated with the quantization of multi-centered black holes are singlets under $I_{3}$. As a result the functions $g_{\text {ref }}$ and consequently $H$ are independent of the parameter $t$ conjugate to $I_{3}$. Thus the only possible source of $t$ dependence is in the index for 'pure Higgs states'. This motivates the conjecture that the Dolbeault polynomial $\widetilde{Q}(\gamma ; y, t)$ for a general quiver $\mathcal{Q}(\gamma)$ is given by the same formula as in section 1 with the replacement $\Omega_{\mathrm{ref}}^{\mathrm{S}}(\alpha / m) \rightarrow \widetilde{\Omega}_{\mathrm{ref}}^{\mathrm{S}}\left(\alpha / m ; t^{m}\right)$ :

$$
\begin{aligned}
\widetilde{Q}(\gamma ; y, t)= & \sum_{m \mid \gamma} \mu(m) m^{-1} \frac{y-y^{-1}}{y^{m}-y^{-m}} \overline{\widetilde{Q}}\left(\gamma / m ; y^{m}, t^{m}\right) \\
\widetilde{\widetilde{Q}}(\gamma ; y, t)= & \sum_{n \geq 1} \sum_{\left\{\alpha_{i} \in \Gamma\right\}, \sum_{i=1}^{n} \alpha_{i}=\gamma} \frac{1}{\operatorname{Aut}\left(\left\{\alpha_{1}, \alpha_{2}, \cdots, \alpha_{n}\right\}\right)} g_{\mathrm{ref}}\left(\alpha_{1}, \alpha_{2}, \cdots, \alpha_{n} ; y\right) \\
& \times \prod_{i=1}^{n}\left\{\sum_{\substack{m_{i} \in \mathbb{Z} \\
m_{i} \mid \alpha_{i}}} \frac{1}{m_{i}} \frac{y-y^{-1}}{y^{m_{i}}-y^{-m_{i}}}\left(\widetilde{\Omega}_{\mathrm{ref}}^{S}\left(\alpha_{i} / m_{i} ; t^{m_{i}}\right)+\widetilde{\Omega}_{\mathrm{scaling}}\left(\alpha_{i} / m_{i} ; y^{m_{i}}, t^{m_{i}}\right)\right)\right\}, \\
\widetilde{\Omega}_{\text {scaling }}(\alpha ; y, t)= & \sum_{\substack{\left\{\beta_{i} \in \Gamma\right\},\left\{m_{i} \in \mathbb{Z}\right\} \\
m_{i} \geq 1, \sum_{i} m_{i} \beta_{i}=\alpha}} H\left(\left\{\beta_{i}\right\} ;\left\{m_{i}\right\} ; y\right) \prod_{i} \widetilde{\Omega}_{\mathrm{ref}}^{S}\left(\beta_{i} ; t^{m_{i}}\right) .
\end{aligned}
$$

Thus we can parametrize $\widetilde{Q}(\gamma ; y, t)$ in terms of the unknown functions $\widetilde{\Omega}_{\text {ref }}^{\mathrm{S}}(\alpha ; t)$. These in turn can be determined if we know the Hirzebruch polynomial $\widetilde{Q}(\gamma ; y, 1 / y)$ given in $(2.14)$ for each $\gamma$. The functions $H\left(\left\{\beta_{i}\right\} ;\left\{m_{i}\right\} ; y\right)$ appearing in $(2.15)$ are the same as before and so need not be determined again. For this reason we have dropped the $y$ dependence in the arguments of $\widetilde{\Omega}_{\text {ref }}^{\mathrm{S}}$ from the outset.

Note that since $\widetilde{Q}(\gamma ; y, t)$ is not protected, we cannot prove that this quantity computed from the Coulomb branch description must match the Higgs branch result. In that sense (2.15) should again be taken as a conjecture for the Dolbeault polynomial of the Higgs branch moduli space. Since all quantities except the $\widetilde{\Omega}_{\mathrm{ref}}^{\mathrm{S}}(\alpha, t)$ 's on the right hand side of this expression are computable, this formula can be tested by independently computing the Dolbeault polynomial of the Higgs branch moduli space for simple quivers.

\subsection{Quiver Poincaré polynomial from the Higgs branch analysis}

The analysis of section 2.2 gives a specific algorithm for computing the Poincaré polynomial of quiver moduli space. In order to test this formula we need an independent determination of the Poincaré polynomial. In this subsection we shall outline the procedure for doing this, generalizing methods used in $[4,19,20]$.

\footnotetext{
${ }^{9}$ This was observed in the context of 2-centered black holes in [2], and elevated in the context of $N=2$ gauge theories to the 'no exotics' conjecture of [10]. As we shall see in the next subsection, this is consistent with the fact that the Coulomb branch accounts for all non-middle cohomology states on the Higgs branch, which necessarily have $p=q$.
} 
The classical moduli space $\mathcal{M}$ on the Higgs branch, or quiver moduli space for brevity, is described by the D-term and F-term conditions (1.1), subject to identifications under $\prod_{\ell} \mathrm{U}\left(N_{\ell}\right)$ gauge transformations. In general, $\mathcal{M}$ is a complex Kähler manifold of complex dimension

$$
d=1-\sum_{\ell} N_{l}^{2}+\sum_{\gamma_{\ell k}>0} \gamma_{\ell k} N_{\ell} N_{k}-f
$$

where $f$ is the number of independent F-term conditions ( $f=0$ for quivers without loop). The BPS states in the supersymmetric quantum mechanics are identified as classes in the total cohomology $H^{*}(\mathcal{M}, \mathbb{Z})$. The Lefschetz operators [25]

$$
J_{+} \cdot h=\omega \wedge h, \quad J_{-}=\omega\left\llcorner h, \quad J_{3} \cdot h=\frac{1}{2}(n-d) h,\right.
$$

where $\omega$ is the Kähler form, $\llcorner=* \wedge *$ where $*$ denotes Hodge star operation, and $n$ is the degree of the differential form $h$, generate an action of $\mathrm{SU}(2)$ on $H^{*}(\mathcal{M}, \mathbb{Z})$ which is identified as $\mathrm{SO}(3)$ rotations in space-time [2]. The refined index $\operatorname{Tr}^{\prime}(-y)^{2 J_{3}}$ of the supersymmetric quantum mechanics on the Higgs branch is given by

$$
Q(\mathcal{M} ; y) \equiv \sum_{p=1}^{2 d} b_{p}(\mathcal{M})(-y)^{p-d}
$$

where $b_{p}(\mathcal{M})=\operatorname{dim} H^{p}(\mathcal{M}, \mathbb{Z})$ is the $p$-th Betti number. In the rest of this subsection we shall restrict our analysis to Abelian quivers, 1.e. with $\mathrm{U}(1)$ gauge groups at each node, deferring a discussion of non-Abelian quivers to section 2.5.

For Abelian quivers, the moduli space of classical vacua $\mathcal{M}$ is obtained by first using part of the F-term constraints to set some of the variables $\phi_{\ell k, \alpha, s s^{\prime}}$ to zero, and then using the standard relation between Kähler quotients and algebro-geometric quotients ${ }^{10}$ to solve the D-term constraints for the remaining variables. As a result, the quiver moduli space $\mathcal{M}$ is generally obtained as a complete intersection of $k$ hypersurfaces inside a product $\mathcal{M}_{\text {amb }}$ of complex projective spaces, corresponding to the F-term constraints which are not trivially solved in the first step. Provided each of the F-term constraints arises as the zero locus of the section of a positive line bundle ${ }^{11}$ over $\mathcal{M}_{\mathrm{amb}}$, it follows from the Lefschetz hyperplane theorem [25] that the Betti numbers $b_{p}(\mathcal{M})$ for $p$ not equal to the complex

\footnotetext{
${ }^{10}$ I.e. the equivalence between, on the one hand, the space of solutions of the D-term constraints modulo the compact gauge group $G=\mathrm{U}(1)^{K}$ and on the other hand, the quotient of the semi-stable locus by the complexified gauge group $G_{\mathbb{C}}=\left(\mathbb{C}^{\times}\right)^{K}$.

${ }^{11}$ We shall also encounter examples where some of the F-term constraints are not given by sections of line bundles with strictly positive curvature - this happens e.g. when some of the constraints are independent of the coordinates of some projective space in $\mathcal{M}_{\mathrm{amb}}$. Even though (2.19) no longer holds for $\mathcal{M}$ directly, one can still regard $\mathcal{M}$ as a product of manifolds for which (2.19) holds. In this case one may parametrize the lack of knowledge of the middle cohomology of each of the manifolds in the product by unknown constants, and determine the $y$ dependence of $Q(\mathcal{M} ; y)$ in terms of these constants. As we shall see in many examples later, knowing the $y$-dependence of $Q(\mathcal{M} ; y)$ will allow us to test the general algorithm for computing $Q(\gamma ; y)$ given in section 1 , and the unknown constants mentioned above will be in one to one correspondence to the constants $\Omega_{\text {ref }}^{\mathrm{S}}(\gamma)$ which appear in the formulæ in section 1 .
} 
dimension $d$ of $\mathcal{M}$ are given in terms of the Betti numbers of the ambient space by

$$
b_{p}(\mathcal{M})= \begin{cases}b_{p}\left(\mathcal{M}_{\mathrm{amb}}\right) & p<d \\ b_{2 d-p}\left(\mathcal{M}_{\mathrm{amb}}\right) & p>d,\end{cases}
$$

leaving the middle cohomology undetermined. The Betti numbers $b_{p}\left(\mathcal{M}_{\mathrm{amb}}\right)$ on the other hand are given by

$$
\sum_{p} b_{p}\left(\mathcal{M}_{\mathrm{amb}}\right) y^{p}=\prod_{\ell=1}^{n} \frac{1-y^{2 a_{\ell}}}{1-y^{2}} .
$$

Eq. (2.19), (2.20) allows us to determine the $y$-dependence of $Q(\mathcal{M} ; y) \equiv$ $(-y)^{-d} \sum_{p} b_{p}(-y)^{p}$, but leaves undetermined the constant term in $Q(\mathcal{M} ; y)$. More generally, the Lefschetz hyperplane theorem ensures that the Hodge numbers $h_{p, q}(\mathcal{M})$ for $p+q \neq d$ are inherited from the ambient space, and therefore that they vanish unless $p=q$. As a result, the Dolbeault polynomial is then a sum

$$
\widetilde{Q}(y, t)=\widetilde{Q}_{\mathrm{amb}}(y)+\widetilde{Q}_{\mathrm{mid}}(t)
$$

of a $t$-independent piece, coming from the cohomology of the ambient projective space, and a $y$-independent piece, coming from the middle cohomology. In general however the quiver moduli space is more complicated, e.g. given by the product of manifolds for each of which (2.21) holds. Our conjecture of section 2.3 is expected to reproduce correctly the Dolbeault polynomial in all such cases.

In order to complete the computation of the Poincaré and Dolbeault polynomials, we need to evaluate the contribution of the middle cohomology. This can be easily obtained from the Euler number $\chi(\mathcal{M})$, equal up to a sign to the value of $Q(\gamma ; y)$ at $y=1$, or from the Hirzebruch polynomial $\chi(\mathcal{M}, v)$, related to the Dolbeault polynomial at $t=1 / y$ via (2.13). Both of them can be computed using the Riemann-Roch theorem, as follows. Suppose as before that the quiver moduli space $\mathcal{M}$ is given by the complete intersection of $k$ hypersurfaces in $\mathcal{M}_{\text {amb }}=\mathbb{P}^{a_{1}-1} \times \cdots \times \mathbb{P}^{a_{n}-1}$. The Riemann-Roch theorem (see e.g. [23]) expresses the Euler characteristics $\chi(\mathcal{M})$ as the coefficient of the top form $J_{1}^{a_{1}-1} \ldots J_{n}^{a_{n}-1}$ in the Laurent expansion around $J_{\ell}=0$ of the rational function

$$
\prod_{\ell=1}^{n}\left(1+J_{\ell}\right)^{a_{\ell}} \prod_{j=1}^{k} \frac{d_{1}^{(j)} J_{1}+\cdots+d_{n}^{(j)} J_{n}}{1+d_{1}^{(j)} J_{1}+\cdots+d_{n}^{(j)} J_{n}},
$$

where $d_{\ell}^{(j)}$ is the degree of the algebraic equation defining the $j$-th hypersurface with respect to the homogeneous coordinates on $\mathbb{P}^{a_{\ell}-1}$. Equivalently, $\chi(\mathcal{M})$ can be obtained as a contour integral around $J_{\ell}=0$,

$$
\chi(\mathcal{M})=\oint \prod_{\ell=1}^{n} \frac{\mathrm{d} J_{\ell}}{2 \pi \mathrm{i}} \prod_{\ell=1}^{n}\left(\frac{1+J_{\ell}}{J_{\ell}}\right)^{a_{\ell}} \prod_{j=1}^{k} \frac{d_{1}^{(j)} J_{1}+\cdots+d_{n}^{(j)} J_{n}}{1+d_{1}^{(j)} J_{1}+\cdots+d_{n}^{(j)} J_{n}} .
$$

For example, for $\mathcal{M}=\mathcal{M}_{\mathrm{amb}}=\mathbb{P}^{a-1}$, the Euler number is given by

$$
\chi\left(\mathbb{P}^{a-1}\right)=\oint \frac{\mathrm{d} J}{2 \pi \mathrm{i}}\left(\frac{1+J}{J}\right)^{a}=\frac{1}{2 \pi \mathrm{i}} \oint \frac{\mathrm{d} x}{(1-x)^{2} x^{a}}=a,
$$


where, in the second equality, we changed variable to $J=x /(1-x)$. For a less trivial example, consider a complete intersection $\mathcal{M}_{n, d_{1}, \ldots d_{k}}$ of $k$ hypersurfaces of degree $d_{1}, \ldots d_{k}$ inside $\mathbb{P}^{n+k}$. Using (2.23), we find that the Euler number is given by

$$
\begin{aligned}
\chi\left(\mathcal{M}_{n, d_{1}, \ldots d_{k}}\right) & =\oint \frac{\mathrm{d} J}{2 \pi \mathrm{i}}\left(\frac{1+J}{J}\right)^{n+k+1} \prod_{j=1}^{k} \frac{d_{j} J}{1+d_{j} J} \\
& =\oint \frac{\mathrm{d} x}{2 \pi \mathrm{i}(1-x)^{2} x^{n+k+1}} \prod_{j=1}^{k} \frac{d_{j} x}{1+\left(d_{j}-1\right) x}
\end{aligned}
$$

where we used the same change of variable. The integral can be computed by introducing the generating function

$$
Z\left(d_{1}, \ldots d_{k} ; z\right) \equiv \sum_{n=0}^{\infty} \chi\left(\mathcal{M}_{n, d_{1}, \ldots d_{k}} ; v\right) z^{n+k}=\oint \frac{\mathrm{d} x}{2 \pi \mathrm{i}(1-x)^{2}} \frac{z^{k}}{x-z} \prod_{j=1}^{k} \frac{d_{j}}{1+\left(d_{j}-1\right) x} .
$$

The integral picks up the residue at $x=z$, leading to the simple result

$$
Z\left(d_{1}, \ldots d_{k} ; z\right)=\frac{1}{(1-z)^{2}} \prod_{j=1}^{k} \frac{d_{j} z}{1+\left(d_{j}-1\right) z} .
$$

As explained in [23], the Hirzebruch polynomial (2.14) can be similarly obtained from the Riemann-Roch theorem, by performing in (2.23) the replacements

$$
\frac{J_{\ell}}{J_{\ell}+1} \rightarrow R_{v}\left(J_{\ell}\right), \quad \frac{d_{1}^{(j)} J_{1}+\cdots+d_{n}^{(j)} J_{n}}{1+d_{1}^{(j)} J_{1}+\cdots+d_{n}^{(j)} J_{n}} \rightarrow R_{v}\left(d_{1}^{(j)} J_{1}+\cdots+d_{n}^{(j)} J_{n}\right),
$$

in (2.23). Here $R_{v}(J)$ is a function of $J$ which reduces to $x=J /(J+1)$ at $v=1$,

$$
R_{v}(J)=\left\{\frac{1-v}{1-e^{-(1-v) J}}+v\right\}^{-1} .
$$

As in (2.24), it is useful to change variable from $J$ to $R=R_{v}(J)$ using

$$
\mathrm{d} J=\frac{\mathrm{d} R}{(1-R)(1-v R)}, \quad J=\frac{1}{1-v} \log \frac{1-v R}{1-R} .
$$

For example, the Hirzebruch polynomial of $\mathbb{P}^{a-1}$ is given by

$$
\chi\left(\mathbb{P}^{a-1} ; v\right)=\oint \frac{\mathrm{d} J}{2 \pi \mathrm{i}\left[R_{v}(J)\right]^{a}}=\oint \frac{\mathrm{d} R}{2 \pi \mathrm{i}(1-R)(1-v R) R^{a}}=\frac{1-v^{a}}{1-v},
$$

in agreement with the fact that $h_{p, p}=1$ for $0 \leq p \leq a-1, h_{p, q}=0$ for $p \neq q$.

Returning to the example discussed in (2.25), the Hirzebruch polynomial of the complete intersection $\mathcal{M}_{n, d_{1}, \ldots d_{k}}$ is given by

$$
\begin{aligned}
\chi\left(\mathcal{M}_{n, d_{1}, \ldots d_{k}} ; v\right) & =\oint \frac{\mathrm{d} J}{2 \pi \mathrm{i}\left[R_{v}(J)\right]^{n+k+1}} \prod_{j=1}^{k} R_{v}\left[d_{j} J\right] \\
& =\oint \frac{\mathrm{d} R}{2 \pi \mathrm{i}(1-R)(1-v R) R^{n+k+1}} \prod_{j=1}^{k} \frac{(1-v R)^{d_{j}}-(1-R)^{d_{j}}}{(1-v R)^{d_{j}}-v(1-R)^{d_{j}}} .
\end{aligned}
$$


The generating function of these polynomials is obtained by summing up the geometric series and picking up the residue at $R=z$, leading to

$$
Z\left(d_{1}, \ldots d_{k} ; z, v\right) \equiv \sum_{n=0}^{\infty} \chi\left(\mathcal{M}_{n, d_{1}, \ldots d_{k}} ; v\right) z^{n+k}=\frac{1}{(1-z)(1-v z)} \prod_{j=1}^{k} \frac{(1-v z)^{d_{j}}-(1-z)^{d_{j}}}{(1-v z)^{d_{j}}-v(1-z)^{d_{j}}}
$$

correcting a misprint in [23], Thm 22.1.1. The manipulations shown in this example will be used in the examples discussed in section 3 onward.

\subsection{Cohomology of quivers without superpotential and HN recursion method}

As discussed in section 2.4, the moduli space for Abelian quivers arises as a complete intersection in an ambient space $\mathcal{M}_{\text {amb }}$ given by a product of complex projective spaces, whose cohomology is easily computed via the Lefschetz hyperplane and Riemann-Roch theorems. For a generic non-Abelian quiver, the situation is more complicated, since the classical moduli space $\mathcal{M}$ is defined by the solution to D-term and F-term equations (1.1) inside the space $\mathbb{C}^{N}$ parametrized by all the complex variables $\phi_{\ell k, \alpha, s s^{\prime}}$, quotiented by the action of the compact group $G=\prod_{\ell} \mathrm{U}\left(N_{\ell}\right)$. We denote by $\mathcal{M}_{0}$ the space of solutions to the D-term equations only, quotiented by the action of $G$. Since the F-term equations are gauge invariant, they descend to the quotient, hence $\mathcal{M}$ is a submanifold of $\mathcal{M}_{0}$. The cohomology of $\mathcal{M}$ can in principle be computed from the cohomology of the ambient space $\mathcal{M}_{0}$ using the methods described in section 2.4. In this section we shall describe a general procedure for computing the cohomology of $\mathcal{M}_{0}$.

By the usual equivalence between Kähler quotients and algebro-geometric quotients, $\mathcal{M}_{0}$ is isomorphic to the quotient of the semi-stable locus $\mathcal{S} \subset \mathbb{C}^{N}$ by the action of the complexified gauge group $G_{\mathbb{C}}=\prod_{\ell} G L\left(N_{\ell}, \mathbb{C}\right)[26]$. Recall that the semi-stable locus is defined as the set of points $x$ for which there exists an homogeneous non-constant $G_{\mathbb{C}^{-}}$ invariant polynomial $F_{x}(X)$ such that $F_{x}(x) \neq 0$. Roughly speaking, semi-stable points are those where the complexified gauge group is broken to a finite group. This implies in particular that $\mathcal{M}_{0}$ is a projective variety, which contains $\mathcal{M}$ as a complex submanifold.

The space $\mathcal{M}_{0}$ is, in effect, the classical quiver moduli space when the superpotential is tuned to zero. When the quiver contains closed loops which admit scaling configurations, this space is non-compact, and hence one might wonder whether its Poincaré polynomial is well-defined. Typically, this non-compactness arises due to a certain number of independent variables $\phi_{i}^{\mathrm{NC}}, i=1 \ldots N^{\prime}$ which are allowed to vary over $\mathbb{C}^{N^{\prime}}$ without restrictions, and hence can become arbitrary large consistently with the D-term constraints. For fixed, finite values of $\phi_{i}^{\mathrm{NC}}$, the remaining variables $\phi_{j}^{\mathrm{C}}, j=1 \ldots N-N^{\prime}$ take values in a compact space $\mathcal{M}_{\mathrm{amb}}\left(\phi^{\mathrm{NC}}\right)$, trivially fibered over $\mathbb{C}^{N^{\prime}}$, such that the fiber never degenerates. The Poincaré polynomial $P_{\mathcal{M}_{0}}(y)$ of $\mathcal{M}_{0}$ is then given by the product of the Poincaré polynomial $P_{\mathcal{M}_{\text {amb }}}(y)$ of the compact space $\mathcal{M}_{\mathrm{amb}}\left(\phi^{\mathrm{NC}}\right)$ (evaluated for example at $\phi_{i}^{\mathrm{NC}}=0$ ) and the Poincaré polynomial of the complex plane labelled by the free variables. For the latter we have $b_{0}=1$ and $b_{p}=0$ for $p>0$, and hence the Poincaré polynomial is 1 . Thus,

$$
Q\left(\mathcal{M}_{0} ; y\right) \equiv(-y)^{-d_{0}} P_{\mathcal{M}_{0}}(-y)=(-y)^{-d_{0}} P_{\mathcal{M}_{\mathrm{amb}}}(-y)=(-y)^{-d_{0}+d_{E}} Q\left(\mathcal{M}_{\mathrm{amb}} ; y\right),
$$


where $d_{0}$ and $d_{E}$ are complex dimensions of $\mathcal{M}_{0}$ and $\mathcal{M}_{\text {amb }}$ respectively. $Q\left(\mathcal{M}_{\mathrm{amb}} ; y\right)$ is invariant under $y \rightarrow 1 / y$ but in general $Q\left(\mathcal{M}_{0} ; y\right)$ is not invariant under $y \rightarrow 1 / y$, since Poincaré duality does not hold for non-compact spaces. In the absence of scaling solutions (in particular, for quivers without loops), the moduli space $\mathcal{M}_{0}$ is compact, and this issue does not arise.

To compute $Q\left(\mathcal{M}_{0} ; y\right)$, we shall apply the Harder-Narasimhan (HN) recursion method. This method was originally established for stable vector bundles over Riemann surfaces $[27,28]$ and recently applied to semi-stable sheaves over rational complex surfaces [29]. For arbitrary quivers without oriented closed loops, the HN recursion method was developed by Reineke, culminating in a general formula for the Poincaré polynomial of the quiver moduli space with any primitive dimension vector [30]. The method was later generalized to quivers with oriented closed loops but vanishing superpotential by [31]. We find strong evidence that the HN recursion method does indeed produce the cohomology of the ambient space $\mathcal{M}_{0}$.

Instead of explaining the logic behind the method, we shall just give the algorithm that derives from it. We introduce the following notations: for the quiver $\mathcal{Q}(\gamma)$ associated to the charge vector $\gamma$ we define $Q_{0}(\gamma ; y)=Q\left(\mathcal{M}_{0} ; y\right)$, and, as in $(1.4), \bar{Q}_{0}(\gamma ; y)$ by

$$
\bar{Q}_{0}(\gamma ; y)=\sum_{m \mid \gamma} m^{-1}\left(y-y^{-1}\right)\left(y^{m}-y^{-m}\right)^{-1} Q_{0}\left(\gamma / m ; y^{m}\right)
$$

From this data we construct a new set of invariants $\mathcal{I}(\gamma ; w)$ via the relations [32-34],

$$
\mathcal{I}(\gamma ; w)=\sum_{\substack{\sum_{i=1}^{k} \alpha_{i}=\gamma, \mu\left(\alpha_{i}\right)=\mu(\gamma)}} \frac{1}{k !} \prod_{i=1}^{k}\left(\frac{\bar{Q}_{0}\left(\alpha_{i} ;-w^{-1}\right)}{w-w^{-1}}\right)
$$

where $\mu(\beta)$ is the 'slope' of the dimension vector $\beta=\sum_{\ell} n_{\ell} \gamma_{\ell}$, defined by

$$
\mu(\beta) \equiv \frac{\sum_{\ell} c_{\ell} n_{\ell}}{\sum_{\ell} n_{\ell}}
$$

Recall that the parameters $c_{\ell}$ are chosen to satisfy $\sum_{\ell} c_{\ell} N_{\ell}=0$, hence $\mu(\gamma)=0$. Finally, for any dimension vector $\beta$ and ordered set of dimension vectors $\left\{\alpha_{i}\right\}$ we define ${ }^{12}$

$$
h(\beta ; w)=\frac{w^{-(\beta, \beta)}}{\prod_{\ell=1}^{K} \prod_{j=1}^{n_{\ell}}\left(1-w^{-2 j}\right)}, \quad \mathcal{F}\left(\left\{\alpha_{i}\right\} ; w\right)=w^{-\sum_{i<j}\left\langle\alpha_{i}, \alpha_{j}\right\rangle} \prod_{i=1}^{k} \mathcal{I}\left(\alpha_{i} ; w\right),
$$

where $(\gamma, \gamma)$ and $\left\langle\gamma, \gamma^{\prime}\right\rangle$ are the Euler form and antisymmetric form defined in (1.3). The HN method states that the invariants $\mathcal{I}(\gamma ; w)$ satisfy the relation $[26-28]$

$$
\mathcal{I}(\gamma ; w)=h(\gamma ; w)-\sum_{k \geq 2} \sum_{\substack{\sum_{i=1}^{k} \alpha_{i}=\gamma, \mu\left(\alpha_{i}\right)>\mu\left(\alpha_{i+1}\right)}} \mathcal{F}\left(\left\{\alpha_{i}\right\} ; w\right) .
$$

\footnotetext{
${ }^{12}$ The function $h(\beta ; w)$ counts the number of quiver representations over finite fields, and is sometimes known as the counting function. The parameter $w$ is related to $y$ by $w=-1 / y$.
} 
We can solve these relations recursively to find $\mathcal{I}(\gamma ; w)$. The solution to the recursion $(2.39)$ is in fact given in terms of the 'counting functions' $h(\beta ; w)$ by [30]

$$
\mathcal{I}(\gamma ; w)=\sum_{\substack{\alpha_{1}+\ldots+\alpha_{k}=\gamma, k \geq 1 \\ \mu\left(\sum_{j=1}^{m} \alpha_{j}\right)>\mu(\gamma), m=1, \ldots, k-1}}(-1)^{k-1} w^{-\sum_{i<j}\left\langle\alpha_{i}, \alpha_{j}\right\rangle} \prod_{j=1}^{k} h\left(\alpha_{j}, w\right) .
$$

Using (2.36) we can then find $\bar{Q}_{0}(\gamma ; y)$ and hence $Q\left(\mathcal{M}_{0} ; y\right)=Q_{0}(\gamma ; y)$. In particular, for a one-node quiver with dimension $N$ and no arrow, we find $Q_{\mathcal{M}_{0}}=1$ if $N=0$ and 0 if $N>1$. This reproduces the fact mentioned in section 2.2 , item 5 , that $\Omega_{\text {ref }}\left(N \gamma_{\ell} ; y\right)=\delta_{1, N}$ for any basis vector $\gamma_{\ell}$ of any quiver.

To further illustrate this method, let us consider the simplest example, the Kronecker quiver with 2 nodes and $\gamma_{12}=a>0$ arrows from node 1 to 2 ,

$$
1 \longrightarrow 2
$$

For $\mathrm{U}(1)$ gauge groups at each node, $\gamma=\gamma_{1}+\gamma_{2}$. Eq. (2.38) then gives

$$
h(\gamma ; w)=\frac{w^{a}}{\left(w-w^{-1}\right)^{2}}, \quad \mathcal{I}\left(\gamma_{\ell} ; w\right)=h\left(\gamma_{\ell} ; w\right)=\frac{1}{w-w^{-1}} .
$$

For $c_{1}>0, c_{2}<0$ we have $\mu\left(\gamma_{2}\right)<0<\mu\left(\gamma_{1}\right)$ and hence the sum over $\left\{\alpha_{i}\right\}$ in (2.39) runs over the ordered pair $\left\{\alpha_{1}, \alpha_{2}\right\}=\left\{\gamma_{1}, \gamma_{2}\right\}$. Eq. (2.39) now gives

$$
\mathcal{I}\left(\gamma_{1}+\gamma_{2} ; w\right)=\left(w^{a}-w^{-a}\right) /\left(w-w^{-1}\right)^{2}
$$

and hence from (2.35) and (2.36) we get

$$
Q_{0}\left(\gamma_{1}+\gamma_{2} ; y\right)=(-1)^{a+1}\left(y^{a}-y^{-a}\right) /\left(y-y^{-1}\right), \quad \text { for } c_{1}>0 .
$$

On the other hand if $c_{1}<0, c_{2}>0$, then the sum over $\left\{\alpha_{i}\right\}$ in (2.39) runs over the ordered pair $\left\{\alpha_{1}, \alpha_{2}\right\}=\left\{\gamma_{2}, \gamma_{1}\right\}$. Eq. (2.39) now gives $\mathcal{I}\left(\gamma_{1}+\gamma_{2} ; w\right)=0$ and hence from (2.35) and $(2.36)$ we get $Q_{0}\left(\gamma_{1}+\gamma_{2} ; y\right)=0$. These results agree with the fact that the quiver moduli space is $\mathbb{P}^{a-1}$ for $c_{1}>0$, and empty otherwise. The Poincaré polynomials for arbitrary dimension vectors $n_{1} \gamma_{1}+n_{2} \gamma_{2}$ (including non-primitive vectors with $\left.\operatorname{gcd}\left(n_{1}, n_{2}\right)>1\right)$ can be obtained by iterating this procedure. Note that in this case there is no distinction between the embedding space $\mathcal{M}_{0}$ and the actual moduli space $\mathcal{M}$.

\section{Abelian three-node quiver}

In this section we shall illustrate the general algorithm outlined in section 1 for the case of a quiver with three nodes, each carrying a $\mathrm{U}(1)$ gauge group, with $(a, b, c)$ arrows as depicted below:

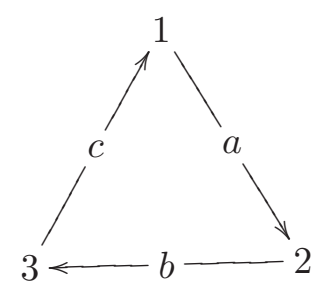


We consider both the the cyclic case, where the arrows form a loop (i.e. $a, b, c$ all of the same sign), and the acyclic case, where the orientation of the arrows does not allow for any loop. The acyclic case has no pure Higgs states, 1.e. $\Omega_{\text {ref }}^{\mathrm{S}}\left(\gamma_{1}+\gamma_{2}+\gamma_{3} ; y\right)=0$. Subject to certain inequalities on the number of arrows, the cyclic case has a non-trivial middle cohomology, which can be exponentially large [4]. It is a special case of the more general cyclic quivers analyzed in section 4 . All of these cases allow us to test the algorithm of section 1.

\subsection{Identifying the contributing collinear configurations}

We start by analyzing the Coulomb branch of the quiver, using the localization techniques of $[6,15]$. According to the prescription of section 2 , we choose a three vectors $\gamma_{1}, \gamma_{2}, \gamma_{3}$ such that

$$
a=\left\langle\gamma_{1}, \gamma_{2}\right\rangle, \quad b=\left\langle\gamma_{2}, \gamma_{3}\right\rangle, \quad c=\left\langle\gamma_{3}, \gamma_{1}\right\rangle,
$$

By permuting the nodes and/or flipping the sign of all $\gamma_{\ell k}$ 's and $c_{\ell}$ 's, operations which leave (1.6) unchanged, we can take the FI parameters to satisfy ${ }^{13}$

$$
c_{1}>0, \quad c_{2}>0, \quad c_{3}=-c_{1}-c_{2}<0,
$$

as in [19]. We shall avoid situations where one of the $c_{\ell}$ 's vanish since this may lie on a wall of marginal stability. Our goal in this subsection will be to identify solutions to (1.6) which contribute to $g_{\mathrm{ref}}(a, b, c ; y) \equiv g_{\mathrm{ref}}\left(\gamma_{1}, \gamma_{2}, \gamma_{3} ; y\right)$ for the above values of the FI parameters. We shall also determine the sign $s(p)$ associated with these solutions via (2.3).

According to section 2, we need to enumerate the permutations $p$ of (123) for which solutions to (1.6) exist. Using symmetry under reversal of the $x$-axis, we only need to examine three permutations: (123), (213) and (132). We first consider the case where none of the multiplicities $a, b, c$ vanish. Let us define

$$
z_{1}=a /\left|x_{1}-x_{2}\right|, \quad z_{2}=b /\left|x_{2}-x_{3}\right|, \quad z_{3}=c /\left|x_{1}-x_{3}\right|,
$$

and

$$
\sigma_{\ell}=\operatorname{sign}\left(x_{\ell+1}-x_{\ell}\right) \quad \text { for } 1 \leq \ell \leq 3, \quad x_{4} \equiv x_{1} .
$$

The equations (1.6) determine $z_{1}, z_{2}$ in terms of $z_{3}$ through

$$
z_{1}=z_{3}+c_{1}, \quad z_{2}=z_{3}+c_{1}+c_{2}
$$

while $z_{3}$ is determined by the requirement that $\left(x_{2}-x_{1}\right)+\left(x_{3}-x_{2}\right)+\left(x_{1}-x_{3}\right)=0$,

$$
f\left(z_{3}\right)=0, \quad f\left(z_{3}\right) \equiv \frac{a \sigma_{1}}{z_{3}+c_{1}}+\frac{b \sigma_{2}}{z_{3}+c_{1}+c_{2}}+\frac{c \sigma_{3}}{z_{3}} .
$$

Thus for any given ordering specified by the choice of $\sigma_{i}= \pm 1$ the solutions of (1.6) are in one-to-one correspondence with the zeros of $f\left(z_{3}\right)$, subject to the inequalities

$$
a /\left(z_{3}+c_{1}\right)>0, \quad b /\left(z_{3}+c_{1}+c_{2}\right)>0, \quad c / z_{3}>0
$$

\footnotetext{
${ }^{13}$ The $c_{i}$ 's are related to the parameters $\theta_{i}$ in [19] (or $\zeta_{i}$ 's in [20]) as $c_{i}=-\theta_{i}=-\zeta_{i}$.
} 
which follow from (3.4) and (3.6). Finally, the determinant of the Hessian of $\hat{W}$ is given by

$$
\operatorname{det} M=-\frac{a b c \sigma_{1} \sigma_{2} \sigma_{3}}{\left(x_{1}-x_{2}\right)^{2}\left(x_{2}-x_{3}\right)^{2}\left(x_{1}-x_{3}\right)^{2}} f^{\prime}\left(z_{3}\right) .
$$

From this we see that any solution to $f\left(z_{3}\right)=0$ contributes a term

$$
(-1)^{a+b+c}\left(y-y^{-1}\right)^{-2} s(p) y^{2 J_{3}(p)}
$$

to the Coulomb index $g_{\mathrm{ref}}(a, b, c ; y)$, where

$$
2 J_{3}(p)=a \sigma_{1}+b \sigma_{2}+c \sigma_{3}, \quad s(p)=\operatorname{sign}\left[-a b c \sigma_{1} \sigma_{2} \sigma_{3} f^{\prime}\left(z_{3}\right)\right] .
$$

A short analysis shows that the conditions (3.8) allow the variable $z_{3}$ to take values in an interval $z_{\min }<z_{3}<z_{\max }$, depending on the signs of $a, b, c$. Within this interval, the function $f\left(z_{3}\right)$ may have several zeros, but since solutions contribute with a sign $s(p)$ proportional to $f^{\prime}\left(z_{3}\right)$, they will cancel in pairs. Thus, a necessary and sufficient condition for the ordering specified by $\sigma_{i}$ to contribute is that $f\left(z_{3}\right)$ should have opposite signs near the two ends of the allowed range, so that an odd number of solutions exist, in which case the sign $s(p)$ will be that of $a b c \sigma_{1} \sigma_{2} \sigma_{3}$, times the sign of $f\left(z_{3}\right)$ near the lower limit of $z_{3}$. Below we tabulate the allowed range of $z_{3}$ as well as the signs of $f\left(z_{3}\right)$ at the two ends of the interval (other combinations of signs of $a, b, c$ are ruled out by the conditions (3.8)):

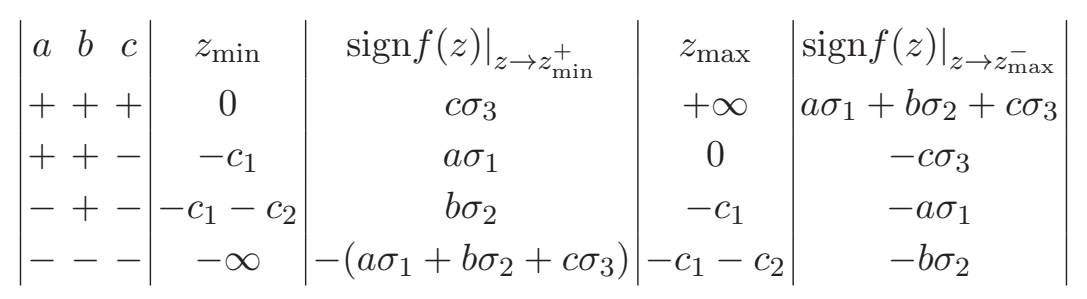

Using this table, it is straightforward to show that the ordering $p(123)=(123)$, corresponding to $\sigma_{1}=\sigma_{2}=1, \sigma_{3}=-1$, contributes whenever

$$
a b>0, \quad c<a+b, \quad s(p)=\operatorname{sign}(a), \quad 2 J_{3}(p)=a+b-c,
$$

while the ordering $p(123)=(213)$, corresponding to $\sigma_{1}=\sigma_{3}=-1, \sigma_{2}=1$ contributes whenever

$$
a c>0, \quad b>a+c, \quad s(p)=-\operatorname{sign}(a), \quad 2 J_{3}(p)=b-a-c,
$$

Finally the ordering $p(123)=(132)$, corresponding to $\sigma_{1}=1, \sigma_{2}=\sigma_{3}=-1$, contributes in four possible cases

$$
\begin{aligned}
& \text { (i) } b, c>0, \quad a>b+c \\
& \text { (ii) } a, b>0, \quad c<0 \\
& \text { (iii) } a, c<0, \quad b>0 \\
& \text { (iv) } b, c<0, \quad a<b+c
\end{aligned}
$$

with $s(p)=-1,2 J_{3}(p)=a-b-c$ in all these cases.

We now consider the case where the multiplicity $a$ vanishes, and $b, c \neq 0$. In that case the equations (1.6) can be solved algebraically. We find that solutions exist only when $b>0, c<0$, and their topology depends on the sign of $\delta=b c_{1}+c c_{2}$. If $\delta>0$, 
the orderings (213) and (132) contribute with signs $s(213)=1, s(132)=-1$, respectively. If $\delta<0$, the orderings (123) and (132) contribute with signs $s(123)=1, s(132)=-1$, respectively. The Coulomb index $g_{\text {ref }}(a, b, c ; y)$ is continuous across the locus $\delta=0$, which corresponds to a wall of threshold stability.

Similarly, if $b=0$ and $a, c \neq 0$, we find that solutions exist only when $a<0, c<0$, and their topology depends on the sign of $\delta=a c_{3}+c c_{2}$. For $\delta>0$, the orderings (213) and (132) contribute, with $s(213)=1, s(132)=-1$, respectively. For $\delta<0$, the orderings (213) and (123) contribute, with $s(213)=1, s(123)=-1$.

Finally, if $c=0$ and $a, b \neq 0$, we find that solutions exist only when $a>0, b>0$, and their topology depends on the sign of $\delta=a c_{3}+b c_{1}$. For $\delta>0$, the orderings (123) and $(213)$ contribute, with $s(123)=1, s(213)=-1$, respectively. For $\delta<0$, the orderings (123) and (132) contribute, with $s(123)=1, s(132)=-1$.

At last, if two of the multiplicities vanish, one of the centers decouples from the other two and the solutions to (1.6) are no longer isolated. This case never arises when discussing non-marginal bound states, as we do in this paper.

We should also discuss the cases where one of the triangle inequalities is saturated. In this case we can still make use of (3.12), but each of the entries where $\left(a \sigma_{1}+b \sigma_{2}+c \sigma_{3}\right)$ appears will need modification when it vanishes since we cannot use this to determine the sign of $f\left(z_{3}\right)$ in appropriate limits. In such cases we need to go back to the expression for $f\left(z_{3}\right)$ given in (3.7) and keep the subleading terms to determine the behaviour of $f\left(z_{3}\right)$ in the $z_{3} \rightarrow \pm \infty$ limit. Take for example the case $c=a+b$ with $a, b, c>0$. In this case $\left(a \sigma_{1}+b \sigma_{2}+c \sigma_{3}\right)$ vanishes for $\sigma_{1}=\sigma_{2}=-\sigma_{3}$. In this case we see from (3.7) that in the $z_{3} \rightarrow \infty$ limit, the sign of $f\left(z_{3}\right)$ is given by that of $c \sigma_{3}$. Since this is the same as the sign of $f\left(z_{3}\right)$ for $z_{3} \rightarrow 0$ we see that this configuration does not contribute to $g_{\text {ref }}$. Similar analysis can be done for all other configurations as well.

\subsection{Three-node quiver without loop}

Let us now consider the case $a<0, b>0, c<0$, corresponding to a three-node quiver without loop. The results of the previous subsection show that only the orderings 213 and 132 contribute, leading to the Laurent polynomial

$$
\begin{aligned}
g_{\text {ref }}\left(\gamma_{1}, \gamma_{2}, \gamma_{3} ; y\right) & =(-1)^{a+b+c}(y-1 / y)^{-2}\left(y^{b-a-c}+y^{a+c-b}-y^{a-c-b}-y^{b+c-a}\right) \\
& =(-1)^{a+b+c} y^{a+c-b-2}\left(1-y^{2}\right)^{-2}\left(1-y^{-2 c}\right)\left(1-y^{2(b-a)}\right) .
\end{aligned}
$$

Since there are no scaling solutions with two centers,

$$
\Omega_{\mathrm{ref}}^{\mathrm{S}}\left(\gamma_{\ell}\right)=1, \quad \Omega_{\mathrm{ref}}^{\mathrm{S}}\left(\gamma_{\ell}+\gamma_{k}\right)=\Omega_{\text {scaling }}\left(\gamma_{\ell}+\gamma_{k} ; y\right)=0, \quad \text { for } 1 \leq \ell<k \leq 3 .
$$

Furthermore for a quiver without closed loop there are also no three centered scaling solutions and hence

$$
\Omega_{\mathrm{ref}}^{\mathrm{S}}\left(\gamma_{1}+\gamma_{2}+\gamma_{3}\right)=\Omega_{\mathrm{scaling}}\left(\gamma_{1}+\gamma_{2}+\gamma_{3} ; y\right)=0 .
$$

eq.(1.4) now gives

$$
Q\left(\gamma_{1}+\gamma_{2}+\gamma_{3} ; y\right)=g_{\mathrm{ref}}\left(\gamma_{1}, \gamma_{2}, \gamma_{3} ; y\right)
$$


On the other hand, the quiver moduli space is described by the D-term equations

$$
\begin{aligned}
& \sum_{\gamma=1}^{|c|}\left|\phi_{13, \gamma}\right|^{2}-\sum_{\alpha=1}^{|a|}\left|\phi_{21, \alpha}\right|^{2}=c_{1} \\
& \sum_{\beta=1}^{b}\left|\phi_{23, \beta}\right|^{2}+\sum_{\alpha=1}^{|a|}\left|\phi_{21, \alpha}\right|^{2}=c_{2} .
\end{aligned}
$$

Since the diagonal U(1) acts trivially on all the fields, they define a manifold $\mathcal{M}$ of complex dimension $|a|+b+|c|-2$, which is a smooth $\mathbb{P}^{|c|-1}$ bundle over $\mathbb{P}^{|a|+b-1}$. The Poincaré polynomial of $\mathcal{M}$ is the product of the Poincaré polynomial of these two projective spaces, in perfect agreement with (3.16).

\subsection{Three node quiver with loop}

Let us now consider a three-node quiver with loop, choosing $a>0, b>0, c>0$. In the case where the triangular inequalities

$$
a<b+c, \quad b<a+c, \quad c<a+b,
$$

hold, the analysis of section 3.1 shows that only the ordering (123) contributes, leading to

$$
g_{\mathrm{ref}}\left(\gamma_{1}, \gamma_{2}, \gamma_{3} ; y\right)=(-1)^{a+b+c}\left(y-y^{-1}\right)^{-2}\left(y^{a+b-c}+y^{c-a-b}\right) .
$$

Unlike (3.16), this is not a Laurent polynomial, as expected since the Coulomb moduli space has scaling regions. Applying the prescription of section 2 we find

$$
Q\left(\gamma_{1}+\gamma_{2}+\gamma_{3} ; y\right)=g_{\mathrm{ref}}\left(\gamma_{1}, \gamma_{2}, \gamma_{3} ; y\right)+\Omega_{\mathrm{ref}}^{\mathrm{S}}\left(\gamma_{1}+\gamma_{2}+\gamma_{3}\right)+H\left(\left\{\gamma_{1}, \gamma_{2}, \gamma_{3}\right\} ;\{1,1,1\} ; y\right) .
$$

The unique choice of $H$, which is even under $y \rightarrow y^{-1}$, vanishes as $y \rightarrow \infty$ and makes the right hand side of (3.23) a polynomial in $y, y^{-1}$ is given by ${ }^{14}$

$$
H\left(\left\{\gamma_{1}, \gamma_{2}, \gamma_{3}\right\} ;\{1,1,1\} ; y\right)=\left\{\begin{array}{l}
-2\left(y-y^{-1}\right)^{-2} \text { for } a+b+c \text { even } \\
\left(y+y^{-1}\right)\left(y-y^{-1}\right)^{-2} \text { for } a+b+c \text { odd }
\end{array}\right.
$$

Substituting these in (3.23) we finally get

$$
\begin{aligned}
& Q\left(\gamma_{1}+\gamma_{2}+\gamma_{3} ; y\right)=\Omega_{\mathrm{ref}}^{\mathrm{S}}\left(\gamma_{1}+\gamma_{2}+\gamma_{3}\right)+ \\
& +(y-1 / y)^{-2} \times \begin{cases}\left(y^{a+b-c}+y^{c-a-b}-2\right), & \text { for } a+b+c \text { even } \\
-\left(y^{a+b-c}+y^{c-a-b}-y-y^{-1}\right), & \text { for } a+b+c \text { odd }\end{cases}
\end{aligned}
$$

On the other hand, if $a, b, c$ are all positive but the triangle inequalities are violated, then it follows from the analysis of section 3.1 that

$$
Q\left(\gamma_{1}+\gamma_{2}+\gamma_{3} ; y\right)=\left(y-y^{-1}\right)^{-2}\left(y^{a+b-c}+y^{c-a-b}-y^{b+c-a}-y^{a-b-c}\right) \quad \text { for } a>b+c
$$

\footnotetext{
${ }^{14}$ Since $H$ is independent of the FI parameters, the result (3.24) can be used for any scaling subquiver of a larger quiver.
} 


$$
\begin{array}{ll}
=\left(y-y^{-1}\right)^{-2}\left(y^{a+b-c}+y^{c-a-b}-y^{a+c-b}-y^{b-a-c}\right) & \text { for } b>a+c \\
=0 \quad \text { for } c>a+b . &
\end{array}
$$

Note that in these cases we have set $\Omega_{\text {ref }}^{\mathrm{S}}\left(\gamma_{1}+\gamma_{2}+\gamma_{3}\right)$ to zero since the Coulomb branch moduli space does not have scaling region. Finally when any one of the inequalities is saturated then we can use either (3.25) or (3.26) since they give the same result.

Let us now compare this result with the cohomology of the Higgs branch. Since the loop allows for a superpotential $W=\sum_{\alpha \beta \gamma} C_{\alpha \beta \gamma} \phi_{12, \alpha} \phi_{23, \beta} \phi_{31, \gamma}$, the moduli space of classical vacua is described by the F-term

$$
\partial_{\phi_{12, \alpha}} W=\partial_{\phi_{23, \beta}} W=\partial_{\phi_{31, \gamma}} W=0
$$

and D-term constraints

$$
\begin{aligned}
& \sum_{\alpha=1}^{a}\left|\phi_{12, \alpha}\right|^{2}-\sum_{\gamma=1}^{c}\left|\phi_{31, \gamma}\right|^{2}=c_{1} \\
& \sum_{\beta=1}^{b}\left|\phi_{23, \beta}\right|^{2}-\sum_{\alpha=1}^{a}\left|\phi_{12, \alpha}\right|^{2}=c_{2} \\
& \sum_{\beta=1}^{c}\left|\phi_{31, \gamma}\right|^{2}-\sum_{\beta=1}^{b}\left|\phi_{23, \beta}\right|^{2}=c_{3}=-c_{1}-c_{2} .
\end{aligned}
$$

As shown in [4], for generic choice of the superpotential the moduli space splits into three different branches, where one of set of chiral multiplets $\phi_{12}, \phi_{23}$ or $\phi_{31}$ vanishes. For the choice of FI terms in (3.3), $\phi_{31}$ vanishes identically, so that the solution to the D-term constraints modulo gauge transformation is given by $\mathbb{P}^{a-1} \times \mathbb{P}^{b-1}$ parametrized by $\phi_{12, \alpha}$ and $\phi_{23, \beta}$ respectively, upon which the F-term conditions $\partial_{\phi_{31, \gamma}} W=0$ impose $c$ bilinear constraints. Thus, $\mathcal{M}$ is a complete intersection in $\mathbb{P}^{a-1} \times \mathbb{P}^{b-1}$. Its cohomology can be computed by the Lefschetz hyperplane theorem, which predicts

$$
\begin{aligned}
Q(\mathcal{M} ; y) & \simeq(-1)^{a+b+c} y^{c-a-b+2}\left(1-y^{2}\right)^{-2}\left(1-y^{2 a}\right)\left(1-y^{2 b}\right) \\
& \simeq(-1)^{a+b+c}(y-1 / y)^{-2} y^{c-a-b}+\mathcal{O}(1)
\end{aligned}
$$

where $\simeq$ denotes equality up to additive constant and positive powers of $y$. This is in agreement with (3.25). The constant $\Omega_{\text {ref }}^{\mathrm{S}}\left(\gamma_{1}+\gamma_{2}+\gamma_{3}\right)$ in (3.25) correspond to the 'pure Higgs states' carrying zero angular momentum.

We shall now obtain the undetermined constant $\Omega_{\text {ref }}^{\mathrm{S}}\left(\gamma_{1}+\gamma_{2}+\gamma_{3}\right)$, by computing the Euler number of $\mathcal{M}$ using the Riemann-Roch theorem. This computation was first carried out in [19], generalizing the analysis of [4]. We shall extend these results by computing the Hirzebruch polynomial (2.14) of the quiver moduli space, which provides finer information on the middle cohomology.

For the three-node with loop of interest in this section, $\mathcal{M}$ is a complete intersection of codimension $c$ in the product $\mathbb{P}^{a-1} \times \mathbb{P}^{b-1}$. After performing the change of variable (2.30), 
we find that the Hirzebruch polynomial is given by

$$
\begin{aligned}
\chi(a, b, c ; v)=\oint & \frac{\mathrm{d} R_{1}}{2 \pi \mathrm{i}\left(1-R_{1}\right)\left(1-v R_{1}\right) R_{1}^{a}} \frac{\mathrm{d} R_{2}}{2 \pi \mathrm{i}\left(1-R_{2}\right)\left(1-v R_{2}\right) R_{2}^{b}} \\
& \times\left(\frac{R_{1}+R_{2}-R_{1} R_{2}(1+v)}{1-R_{1} R_{2} v}\right)^{c}
\end{aligned}
$$

To evaluate this integral, it is useful to construct the partition function

$$
\chi\left(x_{1}, x_{2}, x_{3} ; y\right)=\sum_{a \geq 0, b \geq 0, c \geq 0}(-y)^{-a-b+c+2} \chi\left(a, b, c ; y^{2}\right) x_{1}^{a} x_{2}^{b} x_{3}^{c} .
$$

Summing up the geometric series and computing the contour integral using Cauchy's theorem, we arrive at

$$
\begin{aligned}
& \chi\left(x_{1}, x_{2}, x_{3} ; y\right)= \\
& \quad=\frac{x_{1} x_{2}\left(1-x_{1} x_{2}\right)}{\left(1+x_{1} y\right)\left(1+x_{1} / y\right)\left(1+x_{2} y\right)\left(1+x_{2} / y\right)\left[1-x_{1} x_{2}-x_{2} x_{3}-x_{1} x_{3}-x_{1} x_{2} x_{3}(y+1 / y)\right]} .
\end{aligned}
$$

On the other hand, denoting by $\widehat{Q}\left(x_{1}, x_{2}, x_{3} ; y, t\right)$ the generating function of the Dolbeault polynomial $\widetilde{Q}(a, b, c ; y, t) \equiv \widetilde{Q}\left(\gamma_{1}+\gamma_{2}+\gamma_{3} ; y, t\right)$,

$$
\widehat{Q}\left(x_{1}, x_{2}, x_{3} ; y, t\right)=\sum_{a \geq 0, b \geq 0, c \geq 0}\left(x_{1}\right)^{a}\left(x_{2}\right)^{b}\left(x_{3}\right)^{c} \widetilde{Q}(a, b, c ; y, t),
$$

we find by using (3.26), (2.15) and (3.25)

$$
\begin{aligned}
& \widehat{Q}\left(x_{1}, x_{2}, x_{3} ; y, t\right)=\widehat{Q}^{\mathrm{S}}\left(x_{1}, x_{2}, x_{3} ; t\right) \\
& \quad+\frac{x_{1} x_{2}\left\{1-x_{1} x_{2}+x_{1} x_{2} x_{3}\left(x_{1}+x_{2}+y+y^{-1}\right)\right\}}{\left(1-x_{1} x_{2}\right)\left(1-x_{1} x_{3}\right)\left(1-x_{2} x_{3}\right)\left(1+x_{1} / y\right)\left(1+x_{1} y\right)\left(1+x_{2} / y\right)\left(1+x_{2} y\right)} .
\end{aligned}
$$

Here $\widehat{Q}^{\mathrm{S}}\left(x_{1}, x_{2}, x_{3} ; t\right)$ is the generating function of $\widetilde{\Omega}_{\text {ref }}^{\mathrm{S}}\left(\gamma_{1}+\gamma_{2}+\gamma_{3} ; t\right)$. Note that the $t$ dependence comes only from $\widehat{Q}^{\mathrm{S}}$. Now according to (2.13), at $t=1 / y$ this should reduce to $(-y)^{-d} \chi\left(y^{2}\right)$. Comparing this with (3.32) we find

$$
\widehat{Q}^{\mathrm{S}}\left(x_{1}, x_{2}, x_{3} ; t\right)=\frac{x_{1}^{2} x_{2}^{2} x_{3}^{2}}{\left(1-x_{1} x_{2}\right)\left(1-x_{2} x_{3}\right)\left(1-x_{1} x_{3}\right)\left[1-x_{1} x_{2}-x_{2} x_{3}-x_{1} x_{3}-x_{1} x_{2} x_{3}(t+1 / t)\right]}
$$

It is striking that $\widehat{Q}^{\mathrm{S}}\left(x_{1}, x_{2}, x_{3} ; t\right)$ is symmetric under permutations of $x_{1}, x_{2}, x_{3}$, which implies that the middle cohomology states are robust under wall-crossing. This property at $t=1$ was noticed in [19].

Finally, let us test the HN recursion method described in section 2.5 by computing the cohomology of the quiver moduli space in the absence of a superpotential. We still assume $a, b, c>0$ and $c_{1}>c_{2}>0, c_{3}=-c_{1}-c_{2}<0$. Using the fact that the slopes are ordered according to

$$
\gamma_{3}<\gamma_{2}+\gamma_{3}<\gamma_{1}+\gamma_{3}<\gamma_{1}+\gamma_{2}+\gamma_{3}<\gamma_{2}<\gamma_{1}+\gamma_{2}<\gamma_{1}
$$


we find from $(2.40)$

$$
\begin{aligned}
\mathcal{I}\left(\gamma_{1} ; w\right) & =\mathcal{I}\left(\gamma_{2} ; y\right)=\mathcal{I}\left(\gamma_{3} ; y\right)=1 /\left(w-w^{-1}\right), \\
\mathcal{I}\left(\gamma_{1}+\gamma_{2} ; w\right) & =\left(w^{a}-w^{-a}\right) /\left(w-w^{-1}\right)^{2}, \\
\mathcal{I}\left(\gamma_{2}+\gamma_{3} ; w\right) & =\left(w^{b}-w^{-b}\right) /\left(w-w^{-1}\right)^{2} \\
\mathcal{I}\left(\gamma_{1}+\gamma_{3} ; w\right) & =0
\end{aligned}
$$

and, from (2.36) and (2.40)

$$
\begin{aligned}
Q_{0}\left(\gamma_{1}+\gamma_{2}+\gamma_{3} ; y\right) & =(-1)^{a+b+c} y^{-c}\left(y^{a}-y^{-a}\right)\left(y^{b}-y^{-b}\right) /\left(y-y^{-1}\right)^{2} \\
& =(-1)^{a+b+c} y^{-a-b-c+2}\left(1-y^{2 a}\right)\left(1-y^{2 b}\right)\left(1-y^{2}\right)^{-2}
\end{aligned}
$$

This agrees with the fact that the embedding space $\mathcal{M}_{0}$ is given by $\mathbb{P}^{a-1} \times \mathbb{P}^{b-1} \times \mathbb{C}^{c}[19,20]$.

\section{Cyclic quivers}

We shall now consider a generic cyclic quiver with $K$ nodes, of the form

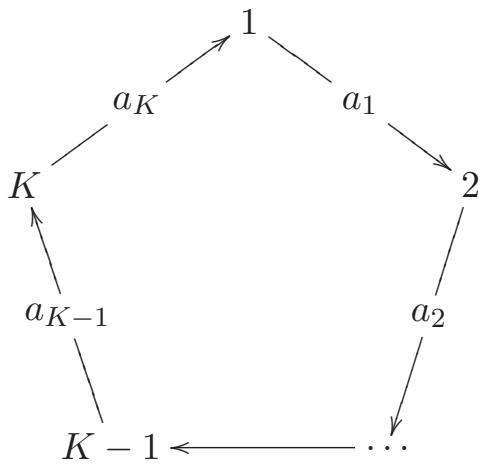

We assume that each node carries a $\mathrm{U}(1)$ factor. We take $\gamma_{\ell(\ell+1)}=a_{\ell}>0$ for $\ell=$ $1, \cdots K-1, \gamma_{K 1}=a_{K}>0$, and choose the FI parameters to satisfy

$$
c_{1}, c_{2}, \cdots c_{K-1}>0, \quad c_{K}<0 .
$$

The Higgs branch of this class of quivers was analyzed in [20]. Since in this case there are no subquivers with closed loops the analysis of both the Coulomb branch and Higgs branch simplifies.

\subsection{Coulomb branch analysis}

The prescription of section 2 yields

$$
Q\left(\gamma_{1}+\cdots+\gamma_{K} ; y\right)=g_{\mathrm{ref}}\left(\gamma_{1}, \cdots \gamma_{K} ; y\right)+H\left(\left\{\gamma_{1}, \cdots \gamma_{K}\right\},\{1, \cdots 1\} ; y\right)+\Omega_{\mathrm{ref}}^{\mathrm{S}}\left(\gamma_{1}+\cdots+\gamma_{K}\right) .
$$

To evaluate the Coulomb index $g_{\mathrm{ref}}\left(\gamma_{1}, \cdots \gamma_{K} ; y\right)$, we need to find the solutions to (1.6) for this system. Extending the procedure of section 3.1, let us define

$$
z_{\ell} \equiv \frac{a_{\ell} \sigma_{\ell}}{x_{\ell+1}-x_{\ell}}, \quad \sigma_{\ell} \equiv \operatorname{sign}\left(x_{\ell+1}-x_{\ell}\right) \quad \text { for } 1 \leq \ell \leq K-1,
$$




$$
z_{K} \equiv \frac{a_{K} \sigma_{K}}{x_{1}-x_{K}}, \quad \sigma_{K} \equiv \operatorname{sign}\left(x_{1}-x_{K}\right)
$$

and rewrite (1.6) as

$$
z_{\ell+1}-z_{\ell}=c_{\ell+1} \quad \text { for } 1 \leq \ell \leq K-2, \quad z_{1}-z_{K}=c_{1}
$$

Since all the $a_{\ell}$ 's are positive, this is also the case of the $z_{\ell}$ 's. Without any loss of generality we can fix $x_{1}=0$. This gives

$$
z_{\ell}=z_{K}+\sum_{k=1}^{\ell} c_{k}, \quad x_{\ell}=\sum_{k=1}^{\ell-1} \frac{a_{k} \sigma_{k}}{z_{k}}
$$

where the only unknown $z_{K}$ is determined by the algebraic equation

$$
f\left(z_{K}\right)=0 \quad \text { where } \quad f\left(z_{K}\right) \equiv \sum_{\ell=1}^{K} \frac{a_{\ell} \sigma_{\ell}}{z_{\ell}} .
$$

Since we assume that all $a_{\ell}(\ell=1 \ldots K)$ and $c_{\ell}(\ell=1 \ldots K-1)$ are positive, the only requirement on the solution of (4.7) is that $z_{K}>0$. For such a solution, the determinant of the Hessian $M_{\ell k}=\partial^{2} \hat{W} / \partial x_{\ell} \partial x_{k}$ for $2 \leq \ell, k \leq K$ evaluates to

$$
\operatorname{det} M=-f^{\prime}\left(z_{K}\right) \prod_{\ell=1}^{K} \frac{a_{\ell} \sigma_{\ell}}{\left(x_{\ell+1}-x_{\ell}\right)^{2}}, \quad x_{K+1} \equiv x_{1} \text {. }
$$

Thus, a solution to $f\left(z_{K}\right)=0$ contributes to $g_{\text {ref }}\left(\gamma_{1}, \cdots \gamma_{K} ; y\right)$ with a sign

$$
s(p)=-\operatorname{sign}\left[f^{\prime}\left(z_{K}\right)\right] \prod_{\ell=1}^{K} \sigma_{\ell} .
$$

In general, the equation $f\left(z_{K}\right)=0$ may have several solutions in the range $0<z_{K}<+\infty$. However, due to (4.9), the contribution of these solutions to $g_{\text {ref }}\left(\gamma_{1}, \cdots \gamma_{K} ; y\right)$ will cancel in pairs. The only possibility for the ordering specified by $\sigma_{i}$ to contribute is that there should be an odd number of solutions. For this we need to ensure that $f\left(z_{K}\right)$ has opposite signs in the two extreme limits: as $z_{K} \rightarrow 0$ and as $z_{K} \rightarrow \infty$. As long as the $c_{\ell}$ 's are not zero we see from (4.6) that all the $z_{\ell}$ 's other than $z_{K}$ remain finite in the $z_{K} \rightarrow 0$ limit and hence $f\left(z_{K}\right)$ approaches $a_{K} \sigma_{K} / z_{K}$. On the other hand as $z_{K} \rightarrow \infty$, we see from (4.6) that all the other $z_{\ell}$ 's also approach infinity keeping the difference $z_{\ell}-z_{K}$ finite and $f\left(z_{K}\right)$ goes as $\sum_{\ell=1}^{K} a_{\ell} \sigma_{\ell} / z_{K}$. Thus (4.7) has an odd number of solutions if

$$
\operatorname{sign}\left[\sum_{\ell=1}^{K} a_{\ell} \sigma_{\ell}\right]=-\sigma_{K}
$$

As indicated above, if there is more than one solution the solutions will cancel in pairs, but the sign of $f^{\prime}\left(z_{K}\right)$ at the uncancelled solution will be the opposite of the sign of $f\left(z_{K}\right)$ as $z_{K} \rightarrow 0$. Since the sign of $f\left(z_{K}\right)$ as $z_{K} \rightarrow 0$ is $\sigma_{K}$, we get, from (4.9),

$$
s(p)=\prod_{\ell=1}^{K-1} \sigma_{\ell}
$$


Using (2.4) we arrive at

$$
g_{\mathrm{ref}}\left(\gamma_{1}, \cdots \gamma_{K} ; y\right)=(-1)^{K-1+\sum_{\ell} a_{\ell}}\left(y-y^{-1}\right)^{-K+1} \sum_{\substack{\sigma_{1}= \pm 1, \sigma_{2}= \pm 1, \cdots \sigma_{K}= \pm 1 \\ \text { sign }\left[\sum_{\ell=1}^{K} a_{\ell} \sigma_{\ell}\right]=-\sigma_{K}}}\left(\prod_{\ell=1}^{K-1} \sigma_{\ell}\right) y^{\sum_{\ell=1}^{K} \sigma_{\ell} a_{\ell}}
$$

Inserting this result in (4.3), we find

$$
\begin{aligned}
& Q\left(\gamma_{1}+\cdots+\gamma_{K} ; y\right)=\Omega_{\mathrm{ref}}^{\mathrm{S}}\left(\gamma_{1}+\cdots+\gamma_{K}\right)+H\left(\left\{\gamma_{1}, \cdots \gamma_{K}\right\},\{1, \cdots 1\} ; y\right) \\
& +(-1)^{K-1+\sum_{\ell} a_{\ell}}\left(y-y^{-1}\right)^{-K+1} \sum_{\substack{\sigma_{1}= \pm 1, \sigma_{2}= \pm 1, \cdots \sigma_{K}= \pm 1 \\
\operatorname{sign}\left[\sum_{\ell=1}^{K} a_{\ell} \sigma_{\ell}\right]=-\sigma_{K}}}\left(\prod_{\ell=1}^{K-1} \sigma_{\ell}\right) y^{\sum_{\ell=1}^{K} \sigma_{\ell} a_{\ell}} .
\end{aligned}
$$

$H\left(\left\{\gamma_{1}, \cdots \gamma_{K}\right\},\{1, \cdots 1\} ; y\right)$ is fixed uniquely by demanding that it is symmetric under $y \rightarrow y^{-1}$, vanishes as $y \rightarrow \infty, 0$, and that $Q\left(\gamma_{1}+\cdots+\gamma_{K} ; y\right)$ is a Laurent polynomial in $y$. It can be obtained for example using the contour integral prescription (2.9), inserting the second line of (4.13) in place of $f(y)$. The constant $\Omega_{\text {ref }}^{\mathrm{S}}\left(\gamma_{1}+\cdots+\gamma_{K}\right)$ appearing in (4.13) can be determined from the Euler characteristics of the Higgs branch, as explained in the next subsections.

In preparation for the analysis of the Higgs branch, let us now try to identify the negative powers of $y$ in (4.13). Firstly, neither $H$ nor $\Omega^{\mathrm{S}}$ contributes negative powers of $y$ in an expansion around $y=0$ since $H$ vanishes as $y \rightarrow 0$ and $\Omega^{\mathrm{S}}$ is $y$-independent constant. To get negative powers of $y$ from the first term on the right hand side of (4.13), we need $\sum_{\ell=1}^{K} \sigma_{\ell} a_{\ell}<0$. Due to the restriction on the $\sigma_{\ell}$ 's in the sum, this implies that $\sigma_{K}=1$. Thus we can express (4.13) as

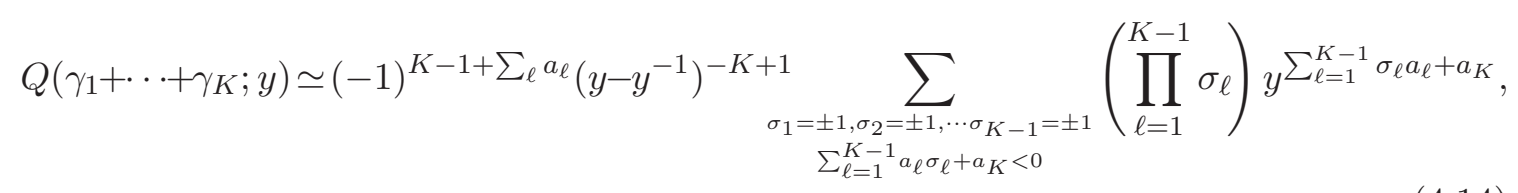

where as usual $\simeq$ denotes equality up to additive constant and positive powers of $y$.

\subsection{Higgs branch analysis}

Now according to the analysis of [20] the moduli space $\mathcal{M}$ of this quiver is a codimension $a_{K}$ complete intersection hypersurface in $\mathbb{P}^{a_{1}-1} \times \cdots \times \mathbb{P}^{a_{K-1}-1}$. Thus the complex dimension of this manifold is given by

$$
d=\sum_{\ell=1}^{K-1} a_{\ell}-a_{K}-(K-1)
$$

By Lefschetz hyperplane theorem, the cohomology of $\mathcal{M}$ coincides with that of $\mathbb{P}^{a_{1}-1} \times \cdots \times \mathbb{P}^{a_{K-1}-1}$ for degree less than $d$. Since the Poincaré polynomial of $\mathbb{P}^{n-1}$ is given by $\left(1-t^{2 n}\right) /\left(1-t^{2}\right)$, we see that the first $d-1$ powers of $t$ of the Poincaré polynomial 
of $\mathcal{M}$ is given by that of $\prod_{\ell=1}^{K-1}\left\{\left(1-t^{2 a_{\ell}}\right) /\left(1-t^{2}\right)\right\}$. Thus the Laurent polynomial associated to $\mathcal{M}$ is given by

$$
\begin{aligned}
Q(\mathcal{M} ; y) & \simeq(-y)^{-\sum_{\ell=1}^{K-1} a_{\ell}+a_{K}+(K-1)} \prod_{\ell=1}^{K-1}\left\{\left(1-y^{2 a_{\ell}}\right) /\left(1-y^{2}\right)\right\} \\
& \simeq(-1)^{-\sum_{\ell=1}^{K} a_{\ell}}\left(y-y^{-1}\right)^{-K+1} y^{-\sum_{\ell=1}^{K-1} a_{\ell}+a_{K}} \prod_{\ell=1}^{K-1}\left(1-y^{2 a_{\ell}}\right),
\end{aligned}
$$

where $\simeq$ denotes equality of terms involving negative powers of $y$. The terms in $Q(\mathcal{M} ; y)$ involving positive powers of $y$ are given by the $y \rightarrow y^{-1}$ symmetry. Now to identify terms in (4.16) involving negative powers of $y$, we can explicitly expand the product $\prod_{\ell=1}^{K-1}\left(1-y^{2 a_{\ell}}\right)$, and pick up those powers of $y$, which when multiplied by $y^{-\sum_{\ell=1}^{K-1} a_{\ell}+a_{K}}$, still gives negative powers of $y$. Thus we get

$$
\begin{aligned}
Q(\mathcal{M} ; y) \simeq & (-1)^{-\sum_{\ell=1}^{K-1} a_{\ell}+a_{K}+K-1}\left(y-y^{-1}\right)^{-K+1} \\
& \times \sum_{\substack{\vec{\sigma} \\
\sigma_{1} a_{1}+\cdots \sigma_{K-1} a_{K-1}+a_{K}<0}}\left(\prod_{\ell=1}^{K-1} \sigma_{\ell}\right) y^{\sigma_{1} a_{1}+\cdots \sigma_{K-1} a_{K-1}+a_{K}},
\end{aligned}
$$

where the sum over $\vec{\sigma}$ runs over all $K-1$ dimensional vectors of the form $( \pm 1, \pm 1, \cdots \pm 1)$ subject to the restriction given above. This is in perfect agreement with (4.14). The agreement between the positive powers of $y$ between $Q(\mathcal{M} ; y)$ and $Q\left(\gamma_{1}+\cdots+\gamma_{K} ; y\right)$ then follows from the $y \rightarrow y^{-1}$ symmetry of both terms. The $H$ in (4.14) ensures that $Q\left(\gamma_{1}+\cdots+\gamma_{K} ; y\right)$, like $Q(\mathcal{M} ; y)$, is a Laurent polynomial in $y$. Finally the $\Omega_{\text {ref }}^{\mathrm{S}}\left(\gamma_{1}+\cdots+\gamma_{K}\right)$ in $(4.14)$ will have to be adjusted so that the constant terms in the expressions for $Q(\mathcal{M} ; y)$ and $Q\left(\gamma_{1}+\cdots+\gamma_{K} ; y\right)$ match.

\subsection{Middle cohomology}

Using the Riemann-Roch theorem summarized in section 2.4, we find that the Hirzebruch polynomial is given by the contour integral

$$
\left.\chi\left(a_{1}, \ldots a_{K} ; v\right)=\oint R_{v}\left(\sum_{\ell=1}^{K-1} J_{\ell}\right)\right]^{a_{K}} \prod_{\ell=1}^{K-1} \frac{\mathrm{d} J_{\ell}}{2 \pi \mathrm{i}\left[R_{v}\left(J_{\ell}\right)\right]^{a_{\ell}}}
$$

where $R_{v}(J)$ has been defined in (2.28). Changing variables from $J_{\ell}$ to $R_{\ell}=R_{v}\left(J_{\ell}\right)$, we find

$$
\chi\left(a_{1}, \ldots a_{K} ; v\right)=\oint\left\{R_{v}\left[\sum_{\ell=1}^{K-1} R_{v}^{-1}\left(R_{\ell}\right)\right]\right\}^{a_{K}} \prod_{\ell=1}^{K-1} \frac{\mathrm{d} R_{\ell}}{2 \pi \mathrm{i}\left(1-R_{\ell}\right)\left(1-v R_{\ell}\right) R_{\ell}^{a_{\ell}}} .
$$

Thus, the partition function, after carrying out the $R_{\ell}$ integrals, is found

$$
\begin{aligned}
\chi\left(x_{1}, \ldots, x_{K} ; y\right) & \equiv \sum_{a_{1}, \cdots a_{K}}(-y)^{-a_{1}-\cdots-a_{K-1}+a_{K}+K-1} \chi\left(a_{1}, \ldots a_{K} ; y^{2}\right) x_{1}^{a_{1}} \ldots x_{K}^{a_{K}} \\
& =\frac{1}{1+x_{K} y R_{y^{2}}\left[\sum_{\ell=1}^{K-1} R_{y^{2}}^{-1}\left(-x_{\ell} / y\right)\right]} \prod_{\ell=1}^{K-1} \frac{x_{\ell}}{\left(1+x_{\ell} / y\right)\left(1+x_{\ell} y\right)}
\end{aligned}
$$


With some work one may express $R_{v}\left[\sum_{\ell=1}^{K-1} R_{v}^{-1}\left(x_{\ell}\right)\right]$ in terms of the $x_{\ell}$,

$$
R_{y^{2}}\left[\sum_{\ell=1}^{K-1} R_{y^{2}}^{-1}\left(-x_{\ell} / y\right)\right]=1+\frac{\left(y-y^{-1}\right) \prod_{\ell=1}^{K-1}\left(1+x_{\ell} / y\right)}{y^{-1} \prod_{\ell=1}^{K-1}\left(1+x_{\ell} y\right)-y \prod_{\ell=1}^{K-1}\left(1+x_{\ell} / y\right)} .
$$

This gives

$$
\chi\left(x_{1}, \ldots, x_{K} ; y\right)=\prod_{\ell=1}^{K-1} \frac{x_{\ell}}{\left(1+x_{\ell} / y\right)\left(1+x_{\ell} y\right)} \frac{y^{-1} \prod_{\ell=1}^{K-1}\left(1+x_{\ell} y\right)-y \prod_{\ell=1}^{K-1}\left(1+x_{\ell} / y\right)}{y^{-1} \prod_{\ell=1}^{K}\left(1+x_{\ell} y\right)-y \prod_{\ell=1}^{K}\left(1+x_{\ell} / y\right)}
$$

Setting $y=1$, we find

$$
\chi\left(x_{1}, \ldots, x_{K} ; 1\right)=\frac{1}{1+x_{K}} \prod_{\ell=1}^{K-1} \frac{x_{\ell}}{\left(1+x_{\ell}\right)^{2}}+D\left(x_{1}, \ldots, x_{K}\right) \prod_{\ell=1}^{K} \frac{x_{\ell}}{1+x_{\ell}},
$$

where

$$
D\left(x_{1}, \ldots, x_{K}\right)=\left(1-\sum_{k=1}^{K} \frac{x_{k}}{1+x_{k}}\right)^{-1} \prod_{\ell=1}^{K} \frac{1}{1+x_{\ell}} .
$$

The function (4.23) is recognized as the generating function of the number $D\left(a_{1}, \ldots a_{K}\right)$ of derangements of a set of $\sum_{\ell=1}^{K} a_{\ell}$ objets of $K$ different types, with $a_{k}$ objects of type $k$ for $k=1 \ldots K$. This partition function was computed in [35] for arbitrary $K$, and its relevance for the counting of pure Higgs states was noted in [19] in the case of 3-node quivers.

On the other hand, the Dolbeault polynomial of the quiver moduli space is given analogously to (4.13) by

$$
\begin{aligned}
& \widetilde{Q}\left(\gamma_{1}+\cdots+\gamma_{K} ; y, t\right)=\widetilde{\Omega}_{\mathrm{ref}}^{\mathrm{S}}\left(\gamma_{1}+\cdots+\gamma_{K} ; t\right)+H\left(\left\{\gamma_{1}, \cdots \gamma_{K}\right\},\{1, \cdots 1\} ; y\right) \\
& +(-1)^{K-1+\sum_{\ell} a_{\ell}}\left(y-y^{-1}\right)^{-K+1} \sum_{\substack{\sigma_{1}= \pm 1, \sigma_{2}= \pm 1, \cdots \sigma_{K}= \pm 1 \\
\operatorname{sign}\left[\sum_{\ell=1}^{K} a_{\ell} \sigma_{\ell}\right]=-\sigma_{K}}}\left(\prod_{\ell=1}^{K-1} \sigma_{\ell}\right) y^{\sum_{\ell=1}^{K} \sigma_{\ell} a_{\ell}}
\end{aligned}
$$

Since the second term $H$ vanishes as $y \rightarrow 0$, we can ignore it for the purpose of determining the non-positive powers of $y$ in $\widetilde{Q}\left(\gamma_{1}+\cdots+\gamma_{K} ; y, t\right)$. The constraint $\operatorname{sign}\left[\sum_{\ell=1}^{K} a_{\ell} \sigma_{\ell}\right]=-\sigma_{K}$ then implies that negative powers of $y$ only come from terms with $\sigma_{K}=1$. Moreover, for such terms we can drop the constraint $\operatorname{sign}\left[\sum_{\ell=1}^{K} a_{\ell} \sigma_{\ell}\right]=-\sigma_{K}$ since terms which violate this constraint carry positive powers of $y$. Thus the generating function for $\widetilde{Q}\left(\gamma_{1}+\cdots+\gamma_{K} ; y, t\right)$ can be written as

$$
\begin{aligned}
\widehat{Q}\left(x_{1}, \cdots x_{K} ; y, t\right): \simeq \widehat{Q}^{\mathrm{S}}\left(x_{1}, \cdots x_{K} ; t\right) \\
\quad+\sum_{\substack{\left\{a_{\ell}\right\} \\
a_{\ell} \geq 0 \forall \ell}}\left(x_{\ell}\right)^{a_{\ell}}(-1)^{K-1+\sum_{\ell} a_{\ell}}\left(y-y^{-1}\right)^{-K+1} \sum_{\sigma_{1}= \pm 1, \sigma_{2}= \pm 1, \cdots \sigma_{K-1}= \pm 1}\left(\prod_{\ell=1}^{K-1} \sigma_{\ell}\right) y^{\sum_{\ell=1}^{K-1} \sigma_{\ell} a_{\ell}} y^{a_{K}} \\
\quad \simeq \widehat{Q}^{\mathrm{S}}\left(x_{1}, \cdots x_{K} ; t\right)+\frac{1}{1+x_{K} y} \prod_{\ell=1}^{K-1} \frac{x_{\ell}}{\left(1+x_{\ell} y\right)\left(1+x_{\ell} / y\right)},
\end{aligned}
$$


where $: \simeq$ denotes equality up to additive positive powers of $y$, and $\widehat{Q}^{\mathrm{S}}$ is the generating function for $\widetilde{\Omega}_{\text {ref }}^{\mathrm{S}}$. Now according to $(2.13)$ we can equate this at $t=1 / y$ to $\chi\left(x_{1}, \ldots, x_{K} ; y\right)$. This gives

$$
\widehat{Q}^{\mathrm{S}}\left(x_{1}, \cdots x_{K} ; 1 / y\right): \simeq \frac{1-y^{2}}{y^{-1} \prod_{\ell=1}^{K}\left(1+x_{\ell} y\right)-y \prod_{\ell=1}^{K}\left(1+x_{\ell} / y\right)} \prod_{\ell=1}^{K} \frac{x_{\ell}}{1+x_{\ell} y}
$$

After expanding in powers of the $x_{\ell}$ 's and picking the coefficient of the monomial $\prod_{\ell} x_{\ell}^{a_{\ell}}$. the right hand side of (4.26) gives the negative and zero powers of $y$ in $\widetilde{\Omega}_{\mathrm{ref}}^{\mathrm{S}}\left(\gamma_{1}+\cdots \gamma_{K} ; 1 / y\right)$. The positive powers of $y$ are found using the $y \rightarrow 1 / y$ symmetry.

In fact, one may compute the complete partition function of $\widetilde{\Omega}_{\mathrm{ref}}^{\mathrm{S}}\left(\gamma_{1}+\cdots \gamma_{K} ; 1 / y\right)$, including positive powers of $y$, by using the prescription (2.9). One can exchange the sums over $a_{\ell}$ with the integral over $u$ as long as $\left|x_{\ell}\right| \ll|u| \forall \ell$. This gives

$$
\begin{aligned}
\widehat{Q}^{\mathrm{S}}\left(x_{1}, \cdots x_{K} ; 1 / y\right)=\oint \frac{\mathrm{d} u}{2 \pi \mathrm{i}} & \frac{(1 / u-u)}{(1-u y)(1-u / y)} \frac{1-u^{2}}{u^{-1} \prod_{\ell=1}^{K}\left(1+x_{\ell} u\right)-u \prod_{\ell=1}^{K}\left(1+x_{\ell} / u\right)} \\
\times & \prod_{\ell=1}^{K} \frac{x_{\ell}}{1+x_{\ell} u},
\end{aligned}
$$

where it is understood that the $u$ integration contour encloses all poles which go to zero as $x_{\ell} \rightarrow 0$ but does not enclose any other poles. The integral can be evaluated as follows:

1. We first make a change of variables from $u \rightarrow 1 / u$. This moves the integration contour so as to enclose the poles at $y$ and $1 / y$.

2. We now deform the integration contour back to the original position, in that process picking up residues at the poles at $u=y$ and $u=1 / y$.

3. We then take the average of the original integral (4.27) and the new result.

At the end of the process one arrives at the result

$$
\begin{aligned}
\widehat{Q}^{\mathrm{S}}\left(x_{1}, \cdots x_{K} ; 1 / y\right)=- & \frac{1}{2} \frac{y-1 / y}{y^{-1} \prod_{\ell=1}^{K}\left(1+x_{\ell} y\right)-y \prod_{\ell=1}^{K}\left(1+x_{\ell} / y\right)} \\
& \times\left[y \prod_{\ell=1}^{K} \frac{x_{\ell}}{1+x_{\ell} y}+y^{-1} \prod_{\ell=1}^{K} \frac{x_{\ell}}{1+x_{\ell} y^{-1}}\right] \\
& -\frac{1}{2} \oint \frac{\mathrm{d} u}{2 \pi \mathrm{i}} \frac{\left(u-u^{-1}\right)^{2}}{(1-u y)(1-u / y)} \prod_{\ell=1}^{K} \frac{x_{\ell}}{\left(1+x_{\ell} u\right)\left(1+x_{\ell} / u\right)} .
\end{aligned}
$$

We can now shrink the last contour to $u=0$, picking up the residues at the poles at $u=-x_{k}$ in that process. This gives

$$
\begin{aligned}
\widehat{Q}^{\mathrm{S}}\left(x_{1}, \cdots x_{K} ; 1 / y\right)=- & \frac{1}{2} \frac{y-1 / y}{y^{-1} \prod_{\ell=1}^{K}\left(1+x_{\ell} y\right)-y \prod_{\ell=1}^{K}\left(1+x_{\ell} / y\right)} \\
& \times\left[y \prod_{\ell=1}^{K} \frac{x_{\ell}}{1+x_{\ell} y}+y^{-1} \prod_{\ell=1}^{K} \frac{x_{\ell}}{1+x_{\ell} y^{-1}}\right]
\end{aligned}
$$




$$
+\frac{1}{2} \sum_{k=1}^{K} \frac{1-x_{k}^{2}}{\left(1+x_{k} / y\right)\left(1+y x_{k}\right)} \prod_{\substack{\ell=1 \ldots K \\ \ell \neq k}} \frac{x_{\ell}}{\left(1-x_{\ell} / x_{k}\right)\left(1-x_{\ell} x_{k}\right)}
$$

This agrees with (3.35) for $K=3$. Like (3.35), eq.(4.29) is also symmetric under the exchange of the $x_{\ell}$ 's reflecting the fact that the single centered index remains invariant under wall crossing. It is worthwhile noting that the poles at $y=-x_{\ell}$ and $y=-1 / x_{\ell}$ precisely cancel between the two terms in (4.29). The partition function (4.29) is also regular at $y=1$, where it reduces to

$$
\widehat{Q}^{\mathrm{S}}\left(x_{1}, \cdots x_{K} ; 1\right)=\frac{\prod_{\ell=1}^{K} \frac{x_{\ell}}{\left(1+x_{\ell}\right)^{2}}}{1-\sum_{k=1}^{K} \frac{x_{k}}{1+x_{k}}}+\frac{1}{2} \sum_{k=1}^{K} \frac{1-x_{k}}{1+x_{k}} \prod_{\substack{\ell=1 \ldots K \\ \ell \neq k}} \frac{x_{\ell}}{\left(1-x_{\ell} / x_{k}\right)\left(1-x_{\ell} x_{k}\right)} .
$$

Using the same techniques as in [19], it is straightforward to extract the asymptotic growth of the index of pure Higgs states $\Omega_{\text {ref }}^{\mathrm{S}}\left(\gamma_{1}+\cdots+\gamma_{K}\right)$ as the arrow multiplicities $a_{\ell}$ are uniformly scaled to infinity. The asymptotics is governed by the pole of the partition function at

$$
\sum_{k=1}^{K} \frac{x_{k}}{1+x_{k}}=1
$$

Setting for simplicity all $a_{\ell}$ equal to $a$, the solution to (4.31) is $x_{k}=1 /(K-1)$, leading to an exponential growth

$$
\Omega_{\mathrm{ref}}^{\mathrm{S}}\left(\gamma_{1}+\cdots+\gamma_{K}\right) \stackrel{a \rightarrow \infty}{\sim} a^{\frac{1-K}{2}}(K-1)^{K a} .
$$

Since $a=\left\langle\gamma_{\ell}, \gamma_{\ell+1}\right\rangle$ scales like the square of the charges, the exponential growth of $\Omega_{\mathrm{ref}}^{\mathrm{S}}$ is consistent with the Bekenstein-Hawking entropy of a macroscopic single-centered black hole.

\section{Quivers with two loops}

So far we have considered quivers for which the links form a single closed loop. In this section we shall apply the general algorithm of section 1 to compute the Poincaré polynomial of quivers with more than one oriented loop.

\subsection{Abelian four-node, two-loop quivers}

We consider the class of Abelian quivers with four nodes and two oriented loops represented below,

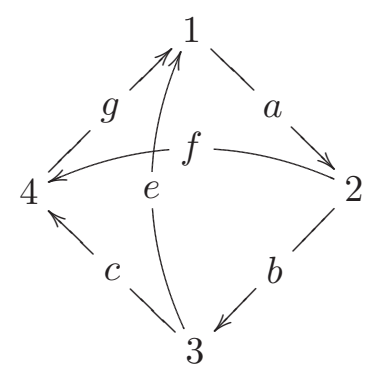


We denote by $\gamma_{1}, \cdots \gamma_{4}$ the charge vectors carried by the four nodes, and by $\gamma_{12}=a$, $\gamma_{23}=b, \gamma_{34}=c, \gamma_{41}=g, \gamma_{31}=e, \gamma_{24}=f$ the multiplicities of arrows, which we assume to be strictly positive. Using (1.4), (1.7), (3.17) and that $\Omega_{\text {ref }}^{\mathrm{S}}\left(\gamma_{\ell}\right)=1$ we get

$$
\begin{aligned}
& Q\left(\gamma_{1}+\gamma_{2}+\gamma_{3}+\gamma_{4} ; y\right)=g_{\mathrm{ref}}\left(\gamma_{1}, \gamma_{2}, \gamma_{3}, \gamma_{4} ; y\right) \\
& \quad+\left\{g_{\mathrm{ref}}\left(\gamma_{1}, \gamma_{2}+\gamma_{3}+\gamma_{4} ; y\right)\left(\Omega_{\mathrm{ref}}^{\mathrm{S}}\left(\gamma_{2}+\gamma_{3}+\gamma_{4}\right)+H\left(\left\{\gamma_{2}, \gamma_{3}, \gamma_{4}\right\} ;\{1,1,1\} ; y\right)\right)+\text { perm }\right\} \\
& \quad+\Omega_{\mathrm{ref}}^{\mathrm{S}}\left(\gamma_{1}+\gamma_{2}+\gamma_{3}+\gamma_{4}\right)+H\left(\left\{\gamma_{1}, \gamma_{2}, \gamma_{3}, \gamma_{4}\right\} ;\{1,1,1,1\} ; y\right) .
\end{aligned}
$$

The coefficients $H\left(\left\{\gamma_{i}, \gamma_{j}, \gamma_{k}\right\} ;\{1,1,1\} ; y\right)$ 's have been determined in section 3 . The coefficient $H\left(\left\{\gamma_{1}, \gamma_{2}, \gamma_{3}, \gamma_{4}\right\} ;\{1,1,1,1\} ; y\right)$ is determined by demanding that the right hand side of (5.2) is a polynomial in $y$ and that $H$ is invariant under $y \rightarrow y^{-1}$ and vanishes as $y \rightarrow \infty$. Instead of trying to solve the problem for a general set of charges, we shall illustrate our algorithm for special choices of the $\gamma_{i j} \equiv\left\langle\gamma_{i}, \gamma_{j}\right\rangle$ and the $c_{i}$ 's. We shall consider two examples:

\subsubsection{Example with only 3-center scaling solutions}

We choose multiplicities ${ }^{15}$

$$
a=3 k, \quad b=4 k, \quad c=7 k, \quad g=4 k, \quad e=5 k, \quad f=4 k,
$$

where $k$ is an arbitrary positive integer, and choose the FI parameters to be

$$
c_{1}=2.1, \quad c_{2}=3, \quad c_{3}=-1.1, \quad c_{4}=-4 .
$$

Since the subquivers 134 and 234 have no closed loops, the corresponding $H$ and $\Omega_{\mathrm{ref}}^{\mathrm{S}}$ must vanish:

$$
\begin{array}{r}
H\left(\left\{\gamma_{1}, \gamma_{3}, \gamma_{4}\right\} ;\{1,1,1\} ; y\right)=H\left(\left\{\gamma_{2}, \gamma_{3}, \gamma_{4}\right\} ;\{1,1,1\} ; y\right)=0 \\
\Omega_{\mathrm{ref}}^{\mathrm{S}}\left(\gamma_{1}+\gamma_{3}+\gamma_{4}\right)=\Omega_{\mathrm{ref}}^{\mathrm{S}}\left(\gamma_{2}+\gamma_{3}+\gamma_{4}\right)=0 .
\end{array}
$$

In contrast, the subquivers 123 and 124 are 3-node quivers with loops of the type analyzed in section 3.3, satisfying the triangular inequalities (3.21). We can therefore borrow the result from (3.24),

$$
\begin{aligned}
& H\left(\left\{\gamma_{1}, \gamma_{2}, \gamma_{3}\right\} ;\{1,1,1\} ; y\right)=-2\left(y-y^{-1}\right)^{-2}, \\
& H\left(\left\{\gamma_{1}, \gamma_{2}, \gamma_{4}\right\} ;\{1,1,1\} ; y\right)=\left\{\begin{array}{l}
\left(y+y^{-1}\right)\left(y-y^{-1}\right)^{-2} \text { for } k \text { odd } \\
-2\left(y-y^{-1}\right)^{-2} \text { for } k \text { even }
\end{array} .\right.
\end{aligned}
$$

The two-center Coulomb indices $g_{\text {ref }}\left(\gamma_{4}, \gamma_{1}+\gamma_{2}+\gamma_{3} ; y\right)$ and $g_{\text {ref }}\left(\gamma_{3}, \gamma_{1}+\gamma_{2}+\gamma_{4} ; y\right)$ can be computed from .(2.5) using $\left\{\hat{c}_{i}\right\}=\left\{c_{4}, c_{1}+c_{2}+c_{3}\right\}=\{-4,4\}$ and $\left\{c_{3}, c_{1}+c_{2}+c_{4}\right\}=\{-1.1,1.1\}$, respectively,

$$
g_{\mathrm{ref}}\left(\gamma_{4}, \gamma_{1}+\gamma_{2}+\gamma_{3} ; y\right)=(-1)^{k+1} \frac{y^{7 k}-y^{-7 k}}{y-y^{-1}}, \quad g_{\mathrm{ref}}\left(\gamma_{3}, \gamma_{1}+\gamma_{2}+\gamma_{4} ; y\right)=0
$$

\footnotetext{
${ }^{15}$ Since scaling the $\gamma_{i j}$ 's by an overall constant $k$ maps a solution to (1.6) to another solution related by simple rescaling of the $x_{i}$ 's without changing their relative order, the computation of $g_{\text {ref }}$ can be done at one go for a family of quivers labelled by differerent values of $k$.
} 
Finally, an explicit analysis of the solutions to (1.6) gives

$$
g_{\mathrm{ref}}\left(\gamma_{1}, \gamma_{2}, \gamma_{3}, \gamma_{4} ; y\right)=(-1)^{k+1}\left(y-y^{-1}\right)^{-3}\left(y^{9 k}-y^{-9 k}+y^{5 k}-y^{-5 k}\right),
$$

with the contribution to $g_{\mathrm{ref}}\left(\gamma_{1}, \gamma_{2}, \gamma_{3}, \gamma_{4} ; y\right)$ arising from the orderings

$$
\{1,2,3,4 ;+\}, \quad\{4,1,2,3 ;-\}
$$

and their reverse (the last entries in (5.9) give the associated signs $s(p)$ ). Substituting these into (5.2) we get

$$
\begin{aligned}
Q\left(\gamma_{1}+\gamma_{2}\right. & \left.+\gamma_{3}+\gamma_{4} ; y\right) \\
= & (-1)^{k+1}\left\{\left(y-y^{-1}\right)^{-3}\left(y^{9 k}-y^{-9 k}+y^{5 k}-y^{-5 k}\right)-2\left(y-y^{-1}\right)^{-3}\left(y^{7 k}-y^{-7 k}\right)\right. \\
& \left.+\left(y-y^{-1}\right)^{-1}\left(y^{7 k}-y^{-7 k}\right) \Omega_{\mathrm{ref}}^{\mathrm{S}}\left(\gamma_{1}+\gamma_{2}+\gamma_{3}\right)\right\} \\
& +H\left(\left\{\gamma_{1}, \gamma_{2}, \gamma_{3}, \gamma_{4}\right\} ;\{1,1,1,1\} ; y\right)+\Omega_{\mathrm{ref}}^{\mathrm{S}}\left(\gamma_{1}+\gamma_{2}+\gamma_{3}+\gamma_{4}\right) .
\end{aligned}
$$

Requiring this to be a polynomial in $y, y^{-1}$, and $H$ to be even under $y \rightarrow y^{-1}$ and vanish as $y \rightarrow \infty$, we get

$$
H\left(\left\{\gamma_{1}, \gamma_{2}, \gamma_{3}, \gamma_{4}\right\} ;\{1,1,1,1\} ; y\right)=0
$$

This gives

$$
\begin{aligned}
Q\left(\gamma_{1}+\gamma_{2}+\gamma_{3}+\gamma_{4} ; y\right)= & \Omega_{\mathrm{ref}}^{\mathrm{S}}\left(\gamma_{1}+\gamma_{2}+\gamma_{3}+\gamma_{4}\right) \\
& +(-1)^{k+1}\left(y^{-7 k+1}+y^{-7 k+3}+\cdots+y^{7 k-1}\right) \\
& \times\left\{\Omega_{\mathrm{ref}}^{\mathrm{S}}\left(\gamma_{1}+\gamma_{2}+\gamma_{3}\right)+\left(y^{-k+1}+y^{-k+3}+\cdots+y^{k-1}\right)^{2}\right\} .
\end{aligned}
$$

The coefficient $\Omega_{\text {ref }}^{\mathrm{S}}\left(\gamma_{1}+\gamma_{2}+\gamma_{3}\right)$ can be determined from the generating function of pure Higgs states given in (3.35) for $(a, b, c)=(3 k, 4 k, 5 k)$ and $t=1$.

The vanishing of $H\left(\left\{\gamma_{1}, \gamma_{2}, \gamma_{3}, \gamma_{4}\right\} ;\{1,1,1,1\} ; y\right)$ indicates that in this case there are no 4 -center scaling solutions. This can be verified by noting that there exist no choice of $\vec{r}_{1}, \cdots \vec{r}_{4}$ for the four centers such that the total angular momentum

$$
\vec{J}=\frac{1}{2} \sum_{i<j} \alpha_{i j} \frac{\vec{r}_{i j}}{\left|\vec{r}_{i j}\right|},
$$

vanishes. As a consequence $\Omega_{\text {ref }}^{\mathrm{S}}\left(\gamma_{1}+\gamma_{2}+\gamma_{3}+\gamma_{4}\right)$ also vanishes. In section 5.1.2 we consider another example where there is a genuine 4-center scaling solution.

Let us now compute $Q(\mathcal{M} ; y)$ by a direct analysis of the cohomology of the quiver moduli space $\mathcal{M}$. For the multiplicities (5.3) and FI parameters (5.4) the D-term equations take the form:

$$
\begin{aligned}
\phi_{12, \alpha}^{*} \phi_{12, \alpha}-\phi_{31, \alpha}^{*} \phi_{31, \alpha}-\phi_{41, \alpha}^{*} \phi_{41, \alpha} & =2.1, \\
-\phi_{12, \alpha}^{*} \phi_{12, \alpha}+\phi_{23, \alpha}^{*} \phi_{23, \alpha}+\phi_{24, \alpha}^{*} \phi_{24, \alpha} & =3, \\
-\phi_{23, \alpha}^{*} \phi_{23, \alpha}+\phi_{31, \alpha}^{*} \phi_{31, \alpha}+\phi_{34, \alpha}^{*} \phi_{34, \alpha} & =-1.1, \\
\phi_{41, \alpha}^{*} \phi_{41, \alpha}-\phi_{24, \alpha}^{*} \phi_{24, \alpha}-\phi_{34, \alpha}^{*} \phi_{34, \alpha} & =-4 .
\end{aligned}
$$


Note that the last equation follows from the first three. In the absence of superpotential, the variables $\left\{\phi^{\mathrm{NC}}\right\}=\left\{\phi_{31, \alpha}, \phi_{41, \alpha}\right\}$ may become arbitrarily large, but for fixed values of those the remaining variables $\left\{\phi^{\mathrm{C}}\right\}=\left\{\phi_{12, \alpha}, \phi_{23, \alpha}, \phi_{24, \alpha}, \phi_{34, \alpha}\right\}$ lie in a compact domain.

Due to the existence of the closed loops 123, 124 and 1234, the generic superpotential takes the form

$$
W=C_{\alpha \beta \gamma}^{(1)} \phi_{12, \alpha} \phi_{23, \beta} \phi_{31, \gamma}+C_{\alpha \beta \gamma}^{(2)} \phi_{12, \alpha} \phi_{24, \beta} \phi_{41, \gamma}+C_{\alpha \beta \gamma \delta}^{(3)} \phi_{12, \alpha} \phi_{23, \beta} \phi_{34, \gamma} \phi_{41, \delta},
$$

where $C^{(i)}$ 's are arbitrary constants. A family of solutions to the F-term and D-term equations can be found by setting:

$$
\begin{aligned}
& \phi_{41, \alpha}=\phi_{31, \alpha}=0, \\
& \phi_{12, \alpha}^{*} \phi_{12, \alpha}=2.1, \quad \phi_{23, \alpha}^{*} \phi_{23, \alpha}=1.1+\phi_{34, \alpha}^{*} \phi_{34, \alpha}, \quad \phi_{34, \alpha}^{*} \phi_{34, \alpha}+\phi_{24, \alpha}^{*} \phi_{24, \alpha}=4, \\
& C_{\alpha \beta \gamma}^{(1)} \phi_{12, \alpha} \phi_{23, \beta}=0, \quad C_{\alpha \beta \delta}^{(2)} \phi_{12, \alpha} \phi_{24, \beta}+C_{\alpha \beta \gamma \delta}^{(3)} \phi_{12, \alpha} \phi_{23, \beta} \phi_{34, \gamma}=0 .
\end{aligned}
$$

Since the Poincaré polynomial remains unchanged under a deformation of the superpotential as long as the moduli space does not become singular or non-compact, we can simplify the problem by choosing the superpotential appropriately. Let us set the coefficients $C_{\alpha \beta \gamma \delta}^{(3)}$ to zero. In that case the last set of equations, $\gamma_{41}=4 k$ in number, can be solved by setting the $\gamma_{24}=4 k$ components $\phi_{24, \alpha}$ to zero. The equations now simplify to

$$
\begin{aligned}
\phi_{41, \alpha}=\phi_{31, \alpha}=\phi_{24, \alpha} & =0, \\
\phi_{12, \alpha}^{*} \phi_{12, \alpha} & =2.1, \quad \phi_{23, \alpha}^{*} \phi_{23, \alpha}=5.1, \quad \phi_{34, \alpha}^{*} \phi_{34, \alpha}=4, \\
C_{\alpha \beta \gamma}^{(1)} \phi_{12, \alpha} \phi_{23, \beta} & =0 .
\end{aligned}
$$

After quotienting by the complexified gauge group $\left(\mathbb{C}^{\times}\right)^{4}$, the moduli space of classical solutions factorizes into a product of $\mathbb{P}^{c-1}$, parametrized by the variables $\phi_{34, \alpha}$, and of a complete intersection of $e$ degree $(1,1)$ hypersurfaces in $\mathbb{P}^{a-1} \times \mathbb{P}^{b-1}$, parametrized by $\phi_{12, \alpha}$ and $\phi_{23, \alpha}$ The cohomology of the complete interesection can be analysed using the Lefschetz hyperplane theorem as explained in section 2.4, or simply borrowed from our previous analysis of 3 -node quivers in $(3.25)$ with $\left(\gamma_{12}, \gamma_{23}, \gamma_{31}\right)=(a, b, e)$. Since the Poincaré polynomial is multiplicative, we arrive at

$$
\begin{aligned}
Q(\mathcal{M} ; y)= & (-1)^{k+1}\left(y^{-7 k+1}+y^{-7 k+3}+\cdots+y^{7 k-1}\right) \\
& \times\left\{\Omega^{\mathrm{S}}\left(\gamma_{1}+\gamma_{2}+\gamma_{3}\right)+\left(y^{-k+1}+y^{-k+3}+\cdots+y^{k-1}\right)^{2}\right\}
\end{aligned}
$$

The value of $\Omega_{\mathrm{ref}}^{\mathrm{S}}\left(\gamma_{1}+\gamma_{2}+\gamma_{3}\right)$ can be determined from the generating function of pure Higgs states in (3.35). Eq.(5.18) is in perfect agreement with (5.12) with $\Omega_{\text {ref }}^{\mathrm{S}}\left(\gamma_{1}+\gamma_{2}+\gamma_{3}+\gamma_{4}\right)=0$.

Finally, let us compare the cohomology of the vacuum moduli space $\mathcal{M}_{0}$ in absence of superpotential with the results of the HN recursion method explained in section 2.5. As noted below (5.14), the variables $\phi_{i}^{\mathrm{NC}}=\phi_{31, \alpha}, \phi_{41, \alpha}$ can vary freely over $\mathbb{C}^{e} \times \mathbb{C}^{g}$, while, for fixed values of those, the remaining variables parametrize the compact manifold $\mathbb{P}^{a-1} \times \mathbb{P}^{b-1} \times \mathbb{P}^{c+f-1}$. The topology of $\mathcal{M}_{0}$ is therefore $\mathbb{P}^{a-1} \times \mathbb{P}^{b-1} \times \mathbb{P}^{c+f-1} \times \mathbb{C}^{e} \times \mathbb{C}^{g}$, with

$$
Q\left(\mathcal{M}_{0} ; y\right)=(-1)^{a+b+c+g+e+f+1} y^{-g-e}\left(y-y^{-1}\right)^{-3}\left(y^{a}-y^{-a}\right)\left(y^{b}-y^{-b}\right)\left(y^{c+f}-y^{-c-f}\right) .
$$


On the other hand, using the fact that the charge vectors are ordered according to

$$
\begin{aligned}
\gamma_{4} & <\gamma_{3}+\gamma_{4}<\gamma_{3}<\gamma_{1}+\gamma_{3}+\gamma_{4}<\gamma_{1}+\gamma_{4}<\gamma_{2}+\gamma_{3}+\gamma_{4}<\gamma_{2}+\gamma_{4}<\gamma_{1}+\gamma_{2}+\gamma_{3}+\gamma_{4} \\
& <\gamma_{1}+\gamma_{2}+\gamma_{4}<\gamma_{1}+\gamma_{3}<\gamma_{2}+\gamma_{3}<\gamma_{1}+\gamma_{2}+\gamma_{3}<\gamma_{1}<\gamma_{1}+\gamma_{2}<\gamma_{2}
\end{aligned}
$$

where we have used the shorthand notation $\alpha<\beta$ to denote $\mu(\alpha)<\mu(\beta)$, the HN recursion method yields

$$
\begin{aligned}
\mathcal{I}\left(\gamma_{1}+\gamma_{2} ; w\right) & =\mathcal{I}\left(\gamma_{1}+\gamma_{3} ; w\right)=\mathcal{I}\left(\gamma_{1}+\gamma_{4} ; w\right)=0, \\
\mathcal{I}\left(\gamma_{2}+\gamma_{3} ; w\right) & =\frac{w^{b}-w^{-b}}{\left(w-w^{-1}\right)^{2}}, \quad \mathcal{I}\left(\gamma_{2}+\gamma_{4}, w\right)=\frac{w^{f}-w^{-f}}{\left(w-w^{-1}\right)^{2}}, \\
\mathcal{I}\left(\gamma_{3}+\gamma_{4} ; w\right) & =\frac{w^{c}-w^{-c}}{\left(w-w^{-1}\right)^{2}}, \quad \mathcal{I}\left(\gamma_{1}+\gamma_{3}+\gamma_{4} ; w\right)=0, \\
\mathcal{I}\left(\gamma_{1}+\gamma_{2}+\gamma_{3} ; w\right) & =w^{e}\left(w-w^{-1}\right)^{-3}\left(w^{b}-w^{-b}\right)\left(w^{a}-w^{-a}\right), \\
\mathcal{I}\left(\gamma_{1}+\gamma_{2}+\gamma_{4} ; w\right) & =w^{g}\left(w-w^{-1}\right)^{-3}\left(w^{f}-w^{-f}\right)\left(w^{a}-w^{-a}\right), \\
\mathcal{I}\left(\gamma_{2}+\gamma_{3}+\gamma_{4} ; w\right) & =\left(w-w^{-1}\right)^{-3}\left(w^{c+f}-w^{-c-f}\right)\left(w^{b}-w^{-b} .\right.
\end{aligned}
$$

Using these results and eq.(2.39), we have, for the total charge vector $\gamma_{1}+\gamma_{2}+\gamma_{3}+\gamma_{4}$,

$$
\begin{aligned}
\mathcal{I}(\gamma ; w)= & h(\gamma ; w)-\mathcal{F}\left(\gamma_{2}, \gamma_{1}, \gamma_{3}+\gamma_{4} ; w\right)-\mathcal{F}\left(\gamma_{1}, \gamma_{2}+\gamma_{3}+\gamma_{4} ; w\right) \\
& -\mathcal{F}\left(\gamma_{1}, \gamma_{2}+\gamma_{3}, \gamma_{4} ; w\right)-\mathcal{F}\left(\gamma_{1}+\gamma_{2}+\gamma_{3}, \gamma_{4} ; w\right)-\mathcal{F}\left(\gamma_{2}, \gamma_{1}, \gamma_{3}, \gamma_{4} ; w\right) \\
& -\mathcal{F}\left(\gamma_{1}, \gamma_{2}+\gamma_{4}, \gamma_{3} ; w\right)-\mathcal{F}\left(\gamma_{1}+\gamma_{2}+\gamma_{4}, \gamma_{3} ; w\right) . \\
= & w^{e+g}\left(w-w^{-1}\right)^{-4}\left(w^{a}-w^{-a}\right)\left(w^{b}-w^{-b}\right)\left(w^{c+f}-w^{-c-f}\right) .
\end{aligned}
$$

Using this and (2.36), we precisely reproduce (5.19). This bolsters our hypothesis that the HN method is applicable to quivers with loops, as long as the superpotential vanishes.

\subsubsection{Example with 4-center scaling solutions}

We now consider a 4-node quiver with the same topology (5.1) but with multiplicities

$$
a=15 k, \quad b=20 k, \quad c=35 k, \quad g=10 k, \quad e=5 k, \quad f=2 k,
$$

where $k$ is a positive integer, and with FI parameters

$$
c_{1}=2, \quad c_{2}=3, \quad c_{3}=-6, \quad c_{4}=1 .
$$

In this case only the subquiver 123 and 124 have closed loops, but the subquiver 124 does not satisfy the triangle inequalities (3.21). Hence we expect $H$ and $\Omega^{\mathrm{S}}$ to vanish for the subquivers 124, 234 and 134 :

$$
\begin{aligned}
H\left(\left\{\gamma_{1}, \gamma_{3}, \gamma_{4}\right\} ;\{1,1,1\} ; y\right) & =H\left(\left\{\gamma_{2}, \gamma_{3}, \gamma_{4}\right\} ;\{1,1,1\} ; y\right)=H\left(\left\{\gamma_{1}, \gamma_{2}, \gamma_{4}\right\} ;\{1,1,1\} ; y\right)=0 \\
\Omega_{\mathrm{ref}}^{\mathrm{S}}\left(\gamma_{1}+\gamma_{3}+\gamma_{4}\right) & =\Omega_{\mathrm{ref}}^{\mathrm{S}}\left(\gamma_{2}+\gamma_{3}+\gamma_{4}\right)=\Omega_{\mathrm{ref}}^{\mathrm{S}}\left(\gamma_{1}+\gamma_{2}+\gamma_{4}\right)=0
\end{aligned}
$$

The analog of (5.6) now has the form:

$$
H\left(\left\{\gamma_{1}, \gamma_{2}, \gamma_{3}\right\} ;\{1,1,1\} ; y\right)=-2\left(y-y^{-1}\right)^{-2} .
$$


Finally an explicit analysis of (1.6) give

$$
\begin{aligned}
g_{\mathrm{ref}}\left(\gamma_{4}, \gamma_{1}+\gamma_{2}+\gamma_{3} ; y\right) & =0, \\
g_{\mathrm{ref}}\left(\gamma_{1}, \gamma_{2}, \gamma_{3}, \gamma_{4} ; y\right) & =(-1)^{k+1}\left(y-y^{-1}\right)^{-3}\left(y^{3 k}-y^{-3 k}\right),
\end{aligned}
$$

with the contribution to $g_{\mathrm{ref}}\left(\gamma_{1}, \gamma_{2}, \gamma_{3}, \gamma_{4} ; y\right)$ arising from the arrangements

$$
\{4,1,2,3 ;+\},
$$

and its reverse. Substituting these into (5.2) we get

$$
\begin{aligned}
Q\left(\gamma_{1}+\gamma_{2}+\gamma_{3}+\gamma_{4} ; y\right)= & (-1)^{k+1}\left(y-y^{-1}\right)^{-3}\left(y^{3 k}-y^{-3 k}\right)+H\left(\left\{\gamma_{1}, \gamma_{2}, \gamma_{3}, \gamma_{4}\right\} ;\{1,1,1,1\} ; y\right) \\
& +\Omega_{\mathrm{ref}}^{\mathrm{S}}\left(\gamma_{1}+\gamma_{2}+\gamma_{3}+\gamma_{4}\right) .
\end{aligned}
$$

The unique choice of $H\left(\left\{\gamma_{1}, \gamma_{2}, \gamma_{3}, \gamma_{4}\right\} ;\{1,1,1,1\} ; y\right)$ consistent with the requirements is

$$
H\left(\left\{\gamma_{1}, \gamma_{2}, \gamma_{3}, \gamma_{4}\right\} ;\{1,1,1,1\} ; y\right)=\left\{\begin{array}{l}
\frac{3}{2} k\left(y-y^{-1}\right)^{-2}\left(y+y^{-1}\right) \text { for } k \text { even } \\
-3 k\left(y-y^{-1}\right)^{-2} \text { for } k \text { odd }
\end{array} .\right.
$$

The fact that $H\left(\left\{\gamma_{1}, \gamma_{2}, \gamma_{3}, \gamma_{4}\right\} ;\{1,1,1,1\} ; y\right)$ does not vanish is consistent with the existence of scaling solutions where all four centers come together (i.e. the existence of four vectors $\vec{r}_{1}, \vec{r}_{2}, \vec{r}_{3}, \vec{r}_{4}$ such that the angular momentum (5.13) vanishes). Substituting this into (5.29) we get

$$
\begin{aligned}
Q & \left(\gamma_{1}+\gamma_{2}+\gamma_{3}+\gamma_{4} ; y\right) \\
& =\left\{\begin{array}{l}
\Omega_{\mathrm{ref}}^{\mathrm{S}}\left(\gamma_{1}+\gamma_{2}+\gamma_{3}+\gamma_{4}\right)-\left(y-y^{-1}\right)^{-3}\left\{y^{3 k}-y^{-3 k}-\frac{3}{2} k\left(y^{2}-y^{-2}\right)\right\} \text { for } k \text { even } \\
\Omega_{\mathrm{ref}}^{\mathrm{S}}\left(\gamma_{1}+\gamma_{2}+\gamma_{3}+\gamma_{4}\right)+\left(y-y^{-1}\right)^{-3}\left\{y^{3 k}-y^{-3 k}-3 k\left(y-y^{-1}\right)\right\} \text { for } k \text { odd }
\end{array}\right.
\end{aligned}
$$

Let us now compute $Q(\mathcal{M} ; y)$ by a direct analysis of the cohomology of the quiver moduli space $\mathcal{M}$. In this case the D-term equations take the form:

$$
\begin{aligned}
\phi_{12, \alpha}^{*} \phi_{12, \alpha}-\phi_{31, \alpha}^{*} \phi_{31, \alpha}-\phi_{41, \alpha}^{*} \phi_{41, \alpha} & =2, \\
-\phi_{12, \alpha}^{*} \phi_{12, \alpha}+\phi_{23, \alpha}^{*} \phi_{23, \alpha}+\phi_{24, \alpha}^{*} \phi_{24, \alpha} & =3, \\
-\phi_{23, \alpha}^{*} \phi_{23, \alpha}+\phi_{31, \alpha}^{*} \phi_{31, \alpha}+\phi_{34, \alpha}^{*} \phi_{34, \alpha} & =-6, \\
\phi_{41, \alpha}^{*} \phi_{41, \alpha}-\phi_{24, \alpha}^{*} \phi_{24, \alpha}-\phi_{34, \alpha}^{*} \phi_{34, \alpha} & =1 .
\end{aligned}
$$

In the absence of a superpotential, the variables $\left\{\phi^{\mathrm{NC}}\right\}=\left\{\phi_{31, \alpha}, \phi_{34, \alpha}, \phi_{24, \alpha}\right\}$ may become arbitrarily large, but for fixed values of those the remaining variables $\left\{\phi^{\mathrm{C}}\right\}=\left\{\phi_{12, \alpha}, \phi_{23, \alpha}, \phi_{41, \alpha}\right\}$ lie in a compact domain. In general however, the superpotential is given by (5.15), where $C^{(i)}$ 's are arbitrary constants. A family of solutions to the F-term and D-term equations can be found by setting

$$
\begin{aligned}
& \phi_{34, \alpha}=\phi_{31, \alpha}=\phi_{24, \alpha}=0 \text {, } \\
& \phi_{12, \alpha}^{*} \phi_{12, \alpha}=3, \quad \phi_{23, \alpha}^{*} \phi_{23, \alpha}=6, \quad \phi_{41, \alpha}^{*} \phi_{41, \alpha}=1,
\end{aligned}
$$




$$
C_{\alpha \beta \gamma}^{(1)} \phi_{12, \alpha} \phi_{23, \beta}=0, \quad C_{\alpha \beta \gamma}^{(2)} \phi_{12, \alpha} \phi_{41, \gamma}=0, \quad C_{\alpha \beta \gamma \delta}^{(3)} \phi_{12, \alpha} \phi_{23, \beta} \phi_{41, \delta}=0 .
$$

Now the variables $\phi_{12, \alpha}, \phi_{23, \alpha}$ and $\phi_{41, \alpha}$ subject to the constraints given in the second line of (5.33), and the identification under the complexified gauge transformations $\left(\mathbb{C}^{\times}\right)^{4}$, describe the product manifold $\mathcal{M}_{\mathrm{amb}}=\mathbb{P}^{a-1} \times \mathbb{P}^{b-1} \times \mathbb{P}^{g-1}$. The constraints in the last line of (5.33) describe a codimension $e+f+c$ submanifold $\mathcal{M}$ inside $\mathcal{M}_{\text {amb. Thus the }}$ quiver moduli space $\mathcal{M}$ has dimension $d=a+b+g-e-f-c=3 k-3$. By repeated use of Lefschetz hyperplane theorem one can argue that the Betti numbers $b_{p}(\mathcal{M})$ for $p<d$ coincide with that of the ambient space $\mathcal{M}_{\mathrm{amb}}$. Thus the negative powers of $y$ in the Laurent polynomial of $\mathcal{M}$ are given by

$$
\begin{aligned}
Q(\mathcal{M} ; y) & \simeq y^{-3 k+3}\left(1+y^{2}+\cdots y^{30 k-2}\right)\left(1+y^{2}+\cdots y^{40 k-2}\right)\left(1+y^{2}+\cdots y^{20 k-2}\right) \\
& \simeq y^{-3 k+3}\left(1-y^{2}\right)^{-3}
\end{aligned}
$$

where $\simeq$ denotes equality of terms involving negative powers of $y$. This is in perfect agreement with (5.31). The $y \rightarrow y^{-1}$ symmetry ensures that the positive powers of $y$ in $Q(\mathcal{M} ; y)$ also agree with that given in (5.31). To determine the constant term $\Omega_{\text {ref }}^{\mathrm{S}}\left(\gamma_{1}+\gamma_{2}+\gamma_{3}+\gamma_{4}\right)$ in (5.31), it suffices to compute the Euler number of the complete intersection manifold described by eq.(5.33). Using the method of section 2.4, we find

$$
\Omega_{\text {ref }}^{\mathrm{S}}\left(\gamma_{1}+\gamma_{2}+\gamma_{3}+\gamma_{4}\right)=(-1)^{k+1} \chi(\mathcal{M})-\left\{\begin{array}{l}
\frac{k}{8}\left(4-9 k^{2}\right) \text { for } k \text { even } \\
\frac{k}{8}\left(9 k^{2}-1\right) \text { for } k \text { odd }
\end{array}\right.
$$

where

$$
\begin{aligned}
\chi(\mathcal{M})= & \oint \prod_{i=1}^{3} \frac{\mathrm{d} J_{i}}{2 \pi \mathrm{i}}\left(\frac{1+J_{1}}{J_{1}}\right)^{15 k}\left(\frac{1+J_{2}}{J_{2}}\right)^{20 k}\left(\frac{1+J_{2}}{J_{3}}\right)^{10 k} \\
& \times\left(\frac{J_{1}+J_{2}}{1+J_{1}+J_{2}}\right)^{5 k}\left(\frac{J_{1}+J_{3}}{1+J_{1}+J_{3}}\right)^{2 k}\left(\frac{J_{1}+J_{2}+J_{3}}{1+J_{1}+J_{2}+J_{3}}\right)^{35 k} .
\end{aligned}
$$

The contour integral can be easily evaluated for any $k$ using the method of section 2.4.

\subsection{A 5-node quiver with nested scaling configurations}

We now consider the 5-node, 2-loop Abelian quiver depicted below,

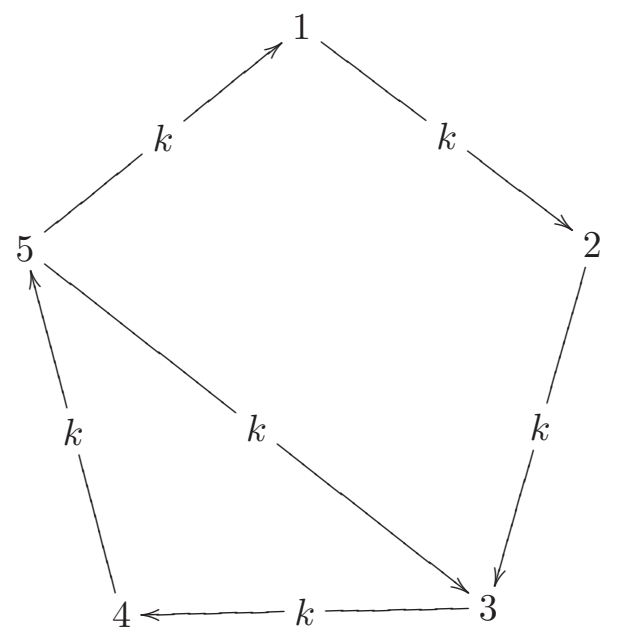


We choose the FI parameters to be

$$
c_{1}=c_{2}=c_{3}=c_{4}=1, \quad c_{5}=-4 .
$$

In this case the nodes 345 form a subquiver satisfying triangle inequality and hence $\Omega_{\mathrm{ref}}^{\mathrm{S}}\left(\gamma_{3}+\right.$ $\left.\gamma_{4}+\gamma_{5}\right)$ and $H\left(\left\{\gamma_{3}, \gamma_{4}, \gamma_{5}\right\},\{1,1,1\} ; y\right)$ are non vanishing. Thus we have

$$
\begin{aligned}
Q\left(\gamma_{1}+\cdots+\gamma_{5} ; y\right)=\Omega_{\mathrm{ref}}^{\mathrm{S}}\left(\gamma_{1}+\cdots+\gamma_{5}\right) \\
\quad+g_{\mathrm{ref}}\left(\gamma_{1}, \gamma_{2}, \gamma_{3}+\gamma_{4}+\gamma_{5} ; y\right)\left[\Omega_{\mathrm{ref}}^{\mathrm{S}}\left(\gamma_{3}+\gamma_{4}+\gamma_{5}\right)+H\left(\left\{\gamma_{3}, \gamma_{4}, \gamma_{5}\right\},\{1,1,1\} ; y\right)\right] \\
\quad+g_{\mathrm{ref}}\left(\gamma_{1}, \cdots \gamma_{5} ; y\right)+H\left(\left\{\gamma_{1}, \cdots \gamma_{5}\right\},\{1, \cdots 1\} ; y\right) \\
\quad+H\left(\left\{\gamma_{1}, \gamma_{2}, \gamma_{3}+\gamma_{4}+\gamma_{5}\right\},\{1,1,1\} ; y\right) \Omega_{\mathrm{ref}}^{\mathrm{S}}\left(\gamma_{3}+\gamma_{4}+\gamma_{5}\right) .
\end{aligned}
$$

The coefficients $g_{\mathrm{ref}}\left(\gamma_{1}, \gamma_{2}, \gamma_{3}+\gamma_{4}+\gamma_{5} ; y\right), H\left(\left\{\gamma_{3}, \gamma_{4}, \gamma_{5}\right\},\{1,1,1\} ; y\right)$ and $H\left(\left\{\gamma_{1}, \gamma_{2}, \gamma_{3}+\right.\right.$ $\left.\left.\gamma_{4}+\gamma_{5}\right\},\{1,1,1\} ; y\right)$ can be read off from the results of section 3 . We have

$$
\begin{gathered}
g_{\mathrm{ref}}\left(\gamma_{1}, \gamma_{2}, \gamma_{3}+\gamma_{4}+\gamma_{5} ; y\right)=(-1)^{k}\left(y-y^{-1}\right)^{-2}\left(y^{k}+y^{-k}\right), \\
\left.H\left(\left\{\gamma_{3}, \gamma_{4}, \gamma_{5}\right\},\{1,1,1\} ; y\right)\right)=\left\{\begin{array}{l}
-2\left(y-y^{-1}\right)^{-2} \text { for } k \text { even } \\
\left(y+y^{-1}\right)\left(y-y^{-1}\right)^{-2} \text { for } k \text { odd }
\end{array}\right. \\
H\left(\left\{\gamma_{1}, \gamma_{2}, \gamma_{3}+\gamma_{4}+\gamma_{5}\right\},\{1,1,1\} ; y\right)=\left\{\begin{array}{l}
-2\left(y-y^{-1}\right)^{-2} \text { for } k \text { even } \\
\left(y+y^{-1}\right)\left(y-y^{-1}\right)^{-2} \text { for } k \text { odd }
\end{array}\right.
\end{gathered}
$$

Finally the contribution to $g_{\text {ref }}\left(\gamma_{1}, \cdots \gamma_{5} ; y\right)$ turns out to arise from the following arrangement of the nodes

$$
\{1,2,3,4,5 ;+\}, \quad\{1,2,5,4,3 ;+\},
$$

and their reverse. This gives

$$
g_{\mathrm{ref}}\left(\gamma_{1}, \cdots \gamma_{5} ; y\right)=\left(y-y^{-1}\right)^{-4}\left(y^{2 k}+y^{-2 k}+2\right)
$$

First consider the case where $k$ is even. In this case (5.39)-(5.42) gives

$$
\begin{aligned}
Q\left(\gamma_{1}+\cdots+\gamma_{5} ; y\right)= & \Omega_{\mathrm{ref}}^{\mathrm{S}}\left(\gamma_{1}+\gamma_{2}+\gamma_{3}+\gamma_{4}+\gamma_{5}\right)+H\left(\left\{\gamma_{1}, \cdots \gamma_{5}\right\},\{1, \cdots 1\} ; y\right) \\
& +\left(y-y^{-1}\right)^{-2}\left(y^{k / 2}-y^{-k / 2}\right)^{2} \Omega_{\mathrm{ref}}^{\mathrm{S}}\left(\gamma_{3}+\gamma_{4}+\gamma_{5}\right) \\
& +\left(y-y^{-1}\right)^{-4}\left(y^{2 k}-2 y^{k}+2-2 y^{-k}+y^{-2 k}\right) .
\end{aligned}
$$

From this we get

$$
H\left(\left\{\gamma_{1}, \cdots \gamma_{5}\right\},\{1, \cdots 1\} ; y\right)=-\frac{k^{2}}{2}\left(y-y^{-1}\right)^{-2},
$$

and hence

$$
\begin{aligned}
Q\left(\gamma_{1}+\cdots+\gamma_{5} ; y\right)= & \Omega_{\mathrm{ref}}^{\mathrm{S}}\left(\gamma_{1}+\gamma_{2}+\gamma_{3}+\gamma_{4}+\gamma_{5}\right) \\
& +\left(y-y^{-1}\right)^{-2}\left(y^{k / 2}-y^{-k / 2}\right)^{2} \Omega_{\mathrm{ref}}^{\mathrm{S}}\left(\gamma_{3}+\gamma_{4}+\gamma_{5}\right)+ \\
& +\left(y-y^{-1}\right)^{-4}\left(y^{2 k}-2 y^{k}+2-2 y^{-k}+y^{-2 k}-\frac{k^{2}}{2}\left(y-y^{-1}\right)^{2}\right) .
\end{aligned}
$$


Next we consider the case where $k$ is odd. In this case (5.39)-(5.42) gives

$$
\begin{aligned}
Q\left(\gamma_{1}+\cdots+\gamma_{5} ; y\right)= & \Omega_{\mathrm{ref}}^{\mathrm{S}}\left(\gamma_{1}+\gamma_{2}+\gamma_{3}+\gamma_{4}+\gamma_{5}\right)+H\left(\left\{\gamma_{1}, \cdots \gamma_{5}\right\},\{1, \cdots 1\} ; y\right) \\
& -\left(y-y^{-1}\right)^{-2}\left(y^{k}+y^{-k}-y-y^{-1}\right) \Omega_{\mathrm{ref}}^{\mathrm{S}}\left(\gamma_{3}+\gamma_{4}+\gamma_{5}\right) \\
& +\left(y-y^{-1}\right)^{-4}\left(y^{2 k}+2+y^{-2 k}-\left(y+y^{-1}\right)\left(y^{k}+y^{-k}\right)\right) .
\end{aligned}
$$

From this we get

$$
H\left(\left\{\gamma_{1}, \cdots \gamma_{5}\right\},\{1, \cdots 1\} ; y\right)=-\frac{k^{2}-1}{2}\left(y-y^{-1}\right)^{-2}
$$

and hence

$$
\begin{aligned}
& Q\left(\gamma_{1}+\cdots+\gamma_{5} ; y\right)=\Omega_{\mathrm{ref}}^{\mathrm{S}}\left(\gamma_{1}+\gamma_{2}+\gamma_{3}+\gamma_{4}+\gamma_{5}\right) \\
& \quad-\left(y-y^{-1}\right)^{-2}\left(y^{k}+y^{-k}-y-y^{-1}\right) \Omega_{\mathrm{ref}}^{\mathrm{S}}\left(\gamma_{3}+\gamma_{4}+\gamma_{5}\right) \\
& \quad+\left(y-y^{-1}\right)^{-4}\left(y^{2 k}+2+y^{-2 k}-\left(y+y^{-1}\right)\left(y^{k}+y^{-k}\right)-\frac{k^{2}-1}{2}\left(y-y^{-1}\right)^{2}\right) .
\end{aligned}
$$

Let us now compare these predictions with the result of direct computation of the cohomology of the quiver moduli space. The D-term equations (1.1) now take the form:

$$
\begin{aligned}
\phi_{12, \alpha}^{*} \phi_{12, \alpha}-\phi_{51, \alpha}^{*} \phi_{51, \alpha} & =1, \\
-\phi_{12, \alpha}^{*} \phi_{12, \alpha}+\phi_{23, \alpha}^{*} \phi_{23, \alpha} & =1, \\
-\phi_{23, \alpha}^{*} \phi_{23, \alpha}+\phi_{34, \alpha}^{*} \phi_{34, \alpha}-\phi_{53, \alpha}^{*} \phi_{53, \alpha} & =1, \\
\phi_{45, \alpha}^{*} \phi_{45, \alpha}-\phi_{34, \alpha}^{*} \phi_{34, \alpha} & =1, \\
-\phi_{45, \alpha}^{*} \phi_{45, \alpha}+\phi_{53, \alpha}^{*} \phi_{53, \alpha}+\phi_{51, \alpha}^{*} \phi_{51, \alpha} & =-4, .
\end{aligned}
$$

In the absence of a superpotential, the variables $\phi_{i}^{\mathrm{NC}}=\left\{\phi_{51, \alpha}, \phi_{53, \alpha}\right\}$ may vary freely but for a fixed value of these variables, the remaining variables live in a compact space. Due to the existence of the oriented closed loops 12345 and 345, the generic superpotential takes the form

$$
W=C_{\alpha \beta \gamma}^{(1)} \phi_{34, \alpha} \phi_{45, \beta} \phi_{53, \gamma}+C_{\alpha \beta \gamma \delta \sigma}^{(2)} \phi_{12, \alpha} \phi_{23, \beta} \phi_{34, \gamma} \phi_{45, \delta} \phi_{51, \sigma},
$$

where $C^{(i)}$ 's are arbitrary constants. A family of solutions to the F-term and D-term conditions can be found by setting:

$$
\begin{aligned}
& \phi_{51, \alpha}=\phi_{53, \alpha}=0, \\
& \phi_{12, \alpha}^{*} \phi_{12, \alpha}=1, \quad \phi_{23, \alpha}^{*} \phi_{23, \alpha}=2, \quad \phi_{34, \alpha}^{*} \phi_{34, \alpha}=3, \quad \phi_{45, \alpha}^{*} \phi_{45, \alpha}=4, \\
& C_{\alpha \beta \gamma}^{(1)} \phi_{34, \alpha} \phi_{45, \beta}=0, \quad C_{\alpha \beta \gamma \delta \sigma}^{(2)} \phi_{12, \alpha} \phi_{23, \beta} \phi_{34, \gamma} \phi_{45, \delta}=0 \text {. }
\end{aligned}
$$

The variables $\phi_{12, \alpha}, \phi_{23, \alpha}, \phi_{34, \alpha}$ and $\phi_{45, \alpha}$ satisfying the constraints in the second line describe a product manifold $\mathbb{P}^{k-1} \times \mathbb{P}^{k-1} \times \mathbb{P}^{k-1} \times \mathbb{P}^{k-1}$ manifold. The first equation in the third line describe a codimension $k$ manifold embedded in the product of the last two $\mathbb{P}^{k-1}$ factors. Let us denote the resulting $k-2$ dimensional manifold by $\overline{\mathcal{M}}$. The 
cohomology of $\overline{\mathcal{M}}$ is in fact identical to that associated with a three node quiver carrying charges $\gamma_{3}, \gamma_{4}$ and $\gamma_{5}$ and is given by (3.25) with $a=b=c=k$. Thus

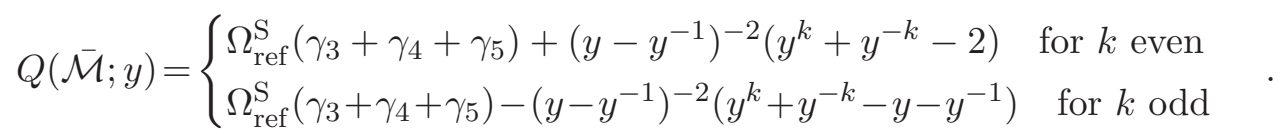

The last equation in the third line of (5.51) now describes a codimension $k$ subspace embedded in $\mathbb{P}^{k-1} \times \mathbb{P}^{k-1} \times \overline{\mathcal{M}}$. The resulting manifold $\mathcal{M}$ has complex dimension $d=2(k-1)+(k-2)-k=2 k-4$, and by the Lefschetz hyperplane theorem its Betti numbers $b_{p}$ are given by those of $\mathbb{P}^{k-1} \times \mathbb{P}^{k-1} \times \overline{\mathcal{M}}$ for $p<d$. This in turn means that the negative powers of $Q(\mathcal{M} ; y)$ are given by

$$
Q(\mathcal{M} ; y) \simeq(-y)^{-2 k+4}\left(1+y^{2}+y^{4}+\cdots+y^{2 k-2}\right)^{2}(-y)^{k-2} Q(\overline{\mathcal{M}} ; y) .
$$

Using (5.52), and throwing away terms involving non-negative powers of $y$, we find

$$
\begin{aligned}
Q(\mathcal{M} ; y) \simeq & (-y)^{-k+2}\left(1-y^{2}\right)^{-2} \Omega_{\mathrm{ref}}^{\mathrm{S}}\left(\gamma_{3}+\gamma_{4}+\gamma_{5}\right) \\
& +\left\{\begin{array}{l}
y^{-k+2}\left(1-y^{2}\right)^{-2}\left(y-y^{-1}\right)^{-2}\left(y^{k}+y^{-k}-2\right) \quad \text { for } k \text { even } \\
y^{-k+2}\left(1-y^{2}\right)^{-2}\left(y-y^{-1}\right)^{-2}\left(y^{k}+y^{-k}-y-y^{-1}\right) \quad \text { for } k \text { odd } .
\end{array}\right.
\end{aligned}
$$

It is easy to see that the negative powers of $y$ in this expression match those in (5.45), (5.48). By $y \rightarrow 1 / y$ symmetry the positive powers of $y$ in the polynomial $Q(\mathcal{M} ; y)$ also match those in (5.48). The constant term is determined by the Euler number of $\mathcal{M}$, which can be computed using the method of section 2.4 ,

$$
\chi(\mathcal{M})=\oint \prod_{i=1}^{4} \frac{\mathrm{d} J_{i}}{2 \pi \mathrm{i}}\left(\frac{\left(1+J_{1}\right)\left(1+J_{2}\right)\left(1+J_{3}\right)\left(1+J_{4}\right)\left(J_{3}+J_{4}\right)\left(J_{1}+J_{2}+J_{3}+J_{4}\right)}{J_{1} J_{2} J_{3} J_{4}\left(1+J_{3}+J_{4}\right)\left(1+J_{1}+J_{2}+J_{3}+J_{4}\right)}\right)^{k} .
$$

Equating (5.55) and (5.48) at $y=1$ allows to determine the pure Higgs state degeneracy $\Omega_{\text {ref }}^{\mathrm{S}}\left(\gamma_{1}+\cdots+\gamma_{5}\right)$.

\section{Non-Abelian quivers}

So far we have only considered quivers for which each node carries a U(1) factor. We shall now analyze some examples of non-Abelian quivers.

\subsection{Rank $(1,1,2)$}

We consider again the 3-node quiver (3.1), but now allow for a $\mathrm{U}(2)$ gauge group at node 3 , keeping $\mathrm{U}(1)$ gauge groups at node 1 and 2 . We assume that the multiplicities $a, b, c$ are positive integers satisfying

$$
a<2 c, \quad b<c, \quad k \equiv a+2 b-2 c>0 .
$$

We choose the FI terms such that

$$
c_{1}>0, \quad c_{1}+c_{2}>0, \quad c_{2}<0, \quad c_{3} \rightarrow 0^{-} .
$$


As mentioned in section 1, when dealing with non-Abelian quivers it is important not to enforce the $y$ independence of $\Omega_{\mathrm{ref}}^{\mathrm{S}}$ until we determine the relevant $H$ 's. Using the fact that the only combination of charges for which the scaling solutions exist are $\gamma_{1}+\gamma_{2}+\gamma_{3}$ and $\gamma_{1}+\gamma_{2}+2 \gamma_{3}$, we can express eqs.(1.4), (1.7) in the form:

$$
\begin{aligned}
& Q\left(\gamma_{1}+\gamma_{2}+2 \gamma_{3} ; y\right)=\frac{1}{2} g_{\mathrm{ref}}\left(\gamma_{1}, \gamma_{2}, \gamma_{3}, \gamma_{3} ; y\right) \Omega_{\mathrm{ref}}^{\mathrm{S}}\left(\gamma_{1} ; y\right) \Omega_{\mathrm{ref}}^{\mathrm{S}}\left(\gamma_{2} ; y\right) \Omega_{\mathrm{ref}}^{\mathrm{S}}\left(\gamma_{3} ; y\right)^{2} \\
& \quad+\frac{1}{2} \frac{y-y^{-1}}{y^{2}-y^{-2}} g_{\mathrm{ref}}\left(\gamma_{1}, \gamma_{2}, 2 \gamma_{3} ; y\right) \Omega_{\mathrm{ref}}^{\mathrm{S}}\left(\gamma_{1} ; y\right) \Omega_{\mathrm{ref}}^{\mathrm{S}}\left(\gamma_{2} ; y\right) \Omega_{\mathrm{ref}}^{\mathrm{S}}\left(\gamma_{3} ; y^{2}\right) \\
& \quad+g_{\mathrm{ref}}\left(\gamma_{1}+\gamma_{2}+\gamma_{3}, \gamma_{3} ; y\right) \Omega_{\mathrm{ref}}^{\mathrm{S}}\left(\gamma_{3} ; y\right) \\
& \quad \times\left[\Omega_{\mathrm{ref}}^{\mathrm{S}}\left(\gamma_{1}+\gamma_{2}+\gamma_{3} ; y\right)+H\left(\left\{\gamma_{1}, \gamma_{2}, \gamma_{3}\right\},\{1,1,1\} ; y\right) \Omega_{\mathrm{ref}}^{\mathrm{S}}\left(\gamma_{1} ; y\right) \Omega_{\mathrm{ref}}^{\mathrm{S}}\left(\gamma_{2} ; y\right) \Omega_{\mathrm{ref}}^{\mathrm{S}}\left(\gamma_{3} ; y\right)\right] \\
& \quad+\Omega_{\mathrm{ref}}^{\mathrm{S}}\left(\gamma_{1}+\gamma_{2}+2 \gamma_{3} ; y\right)+H\left(\left\{\gamma_{1}, \gamma_{2}, \gamma_{3}, \gamma_{3}\right\},\{1,1,1,1\} ; y\right) \Omega_{\mathrm{ref}}^{\mathrm{S}}\left(\gamma_{1} ; y\right) \Omega_{\mathrm{ref}}^{\mathrm{S}}\left(\gamma_{2} ; y\right) \Omega_{\mathrm{ref}}^{\mathrm{S}}\left(\gamma_{3} ; y\right)^{2} \\
& \quad+H\left(\left\{\gamma_{1}, \gamma_{2}, \gamma_{3}\right\},\{1,1,2\} ; y\right) \Omega_{\mathrm{ref}}^{\mathrm{S}}\left(\gamma_{1} ; y\right) \Omega_{\mathrm{ref}}^{\mathrm{S}}\left(\gamma_{2} ; y\right) \Omega_{\mathrm{ref}}^{\mathrm{S}}\left(\gamma_{3} ; y^{2}\right)
\end{aligned}
$$

Using (6.1), (2.5) and the result of section 3.3 we get

$$
g_{\mathrm{ref}}\left(\gamma_{1}+\gamma_{2}+\gamma_{3}, \gamma_{3} ; y\right)=0, \quad g_{\mathrm{ref}}\left(\gamma_{1}, \gamma_{2}, 2 \gamma_{3} ; y\right)=(-1)^{k} \frac{y^{k}+y^{-k}}{\left(y-y^{-1}\right)^{2}} .
$$

Finally to find $g_{\text {ref }}\left(\gamma_{1}, \gamma_{2}, \gamma_{3}, \gamma_{3} ; y\right)$ we label the coordinates of the charges $\gamma_{1}, \gamma_{2}, \gamma_{3}, \gamma_{3}$ by $x_{1}, x_{2}, x_{3}, x_{4}$. Eq. (1.6) now gives

$$
\frac{a}{\left|x_{12}\right|}-\frac{c}{\left|x_{13}\right|}-\frac{c}{\left|x_{14}\right|}=c_{1}, \quad \frac{c}{\left|x_{13}\right|}-\frac{b}{\left|x_{23}\right|}=c_{3}, \quad \frac{c}{\left|x_{14}\right|}-\frac{b}{\left|x_{24}\right|}=c_{3} .
$$

Note that the last two equations, regarded as equations for $x_{3}$ and $x_{4}$ respectively, are identical equations and hence we can try to solve them simultaneously for fixed $x_{1}, x_{2}$. Using translation invariance and reversal symmetry of the $x$ axis we can take $x_{1}=0$, $x_{2}>0$. In the $c_{3} \rightarrow 0$ limit the last two equations in (6.5) give

$$
b\left|x_{a}\right|=c\left|x_{a}-x_{2}\right| \quad \text { for } a=3,4 .
$$

Since $b<c$, this equation has two possible solutions - one solution $x_{\mathrm{m}}$ in the range $x_{1}<x_{a}<x_{2}$, and another $x_{\mathrm{r}}$ in the range $x_{a}>x_{2}$,

$$
0=x_{1}<x_{\mathrm{m}} \equiv \frac{c}{b+c} x_{2}<x_{2}<x_{\mathrm{r}} \equiv \frac{c}{c-b} x_{2}
$$

Consider now the solution where $n_{A}$ of the $\gamma_{3}$ 's sit at $x_{\mathrm{m}}$ and $n_{B}$ of the $\gamma_{3}$ sit at $x_{\mathrm{r}}$. Here $n_{A}, n_{B}=0,1,2$ subject to the restriction $n_{A}+n_{B}=2$. Substituting the corresponding values of $x_{i}$ into the first equation in (6.5) we get

$$
c_{1} x_{2}=a-b\left(n_{A}-n_{B}\right)-c\left(n_{A}+n_{B}\right) .
$$

Since $c_{1}>0$ and $x_{2}>0$, the right hand side of (6.8) must be positive. The condition $a<2 c$ in (6.1) now shows that this is possible only for the choice $n_{A}=0, n_{B}=2$. Furthermore 
one finds that for this case $s(p)=1$ [15]. Thus the contribution to $g_{\mathrm{ref}}\left(\gamma_{1}, \gamma_{2}, \gamma_{3}, \gamma_{3} ; y\right)$ comes from the permutation $\{1,2,3,4 ;+\}$ and its reverse. ${ }^{16}$ This gives

$$
g_{\mathrm{ref}}\left(\gamma_{1}, \gamma_{2}, \gamma_{3}, \gamma_{3} ; y\right)=(-1)^{k+1}\left(y-y^{-1}\right)^{-3}\left(y^{k}-y^{-k}\right),
$$

Eq. (6.3) now gives

$$
\begin{aligned}
& Q\left(\gamma_{1}+\gamma_{2}+2 \gamma_{3} ; y\right)=\Omega_{\mathrm{ref}}^{\mathrm{S}}\left(\gamma_{1}+\gamma_{2}+2 \gamma_{3} ; y\right) \\
& +\left\{H\left(\left\{\gamma_{1}, \gamma_{2}, \gamma_{3}\right\},\{1,1,2\} ; y\right)+\frac{(-1)^{k}}{2\left(y-y^{-1}\right)^{2}} \frac{y^{k}+y^{-k}}{y+y^{-1}}\right\} \Omega_{\mathrm{ref}}^{\mathrm{S}}\left(\gamma_{1} ; y\right) \Omega_{\mathrm{ref}}^{\mathrm{S}}\left(\gamma_{2} ; y\right) \Omega_{\mathrm{ref}}^{\mathrm{S}}\left(\gamma_{3} ; y^{2}\right) \\
& +\left\{H\left(\left\{\gamma_{1}, \gamma_{2}, \gamma_{3}, \gamma_{4}\right\}\{1,1,1,1\} ; y\right)+\frac{1}{2}(-1)^{k+1}\left(y-y^{-1}\right)^{-3}\left(y^{k}-y^{-k}\right)\right\} \\
& \quad \times \Omega_{\mathrm{ref}}^{\mathrm{S}}\left(\gamma_{1} ; y\right) \Omega_{\mathrm{ref}}^{\mathrm{S}}\left(\gamma_{2} ; y\right) \Omega_{\mathrm{ref}}^{\mathrm{S}}\left(\gamma_{3} ; y\right)^{2}
\end{aligned}
$$

Requiring that the coefficients of $\Omega_{\mathrm{ref}}^{\mathrm{S}}\left(\gamma_{1} ; y\right) \Omega_{\mathrm{ref}}^{\mathrm{S}}\left(\gamma_{2} ; y\right) \Omega_{\mathrm{ref}}^{\mathrm{S}}\left(\gamma_{3} ; y^{2}\right)$ and $\Omega_{\mathrm{ref}}^{\mathrm{S}}\left(\gamma_{1} ; y\right) \Omega_{\mathrm{ref}}^{\mathrm{S}}\left(\gamma_{2} ; y\right)$ $\Omega_{\text {ref }}^{\mathrm{S}}\left(\gamma_{3} ; y\right)^{2}$ be polynomials in $y, y^{-1}$ we get

$$
\begin{aligned}
& H\left(\left\{\gamma_{1}, \gamma_{2}, \gamma_{3}, \gamma_{3}\right\}\{1,1,1,1\} ; y\right)= \begin{cases}\frac{1}{4} k\left(y-y^{-1}\right)^{-2}\left(y+y^{-1}\right) & \text { for } k \text { even } \\
-\frac{1}{2} k\left(y-y^{-1}\right)^{-2} & \text { for } k \text { odd }\end{cases} \\
& H\left(\left\{\gamma_{1}, \gamma_{2}, \gamma_{3}\right\},\{1,1,2\} ; y\right) \\
& \quad= \begin{cases}\frac{1}{4}\left(y-y^{-1}\right)^{-2}\left(y+y^{-1}\right)^{-1}\left\{-\left(y+y^{-1}\right)^{2}+(-1)^{k / 2}\left(y-y^{-1}\right)^{2}\right\} & \text { for } k \text { even } \\
\frac{1}{2}\left(y-y^{-1}\right)^{-2} & \text { for } k \text { odd }\end{cases}
\end{aligned}
$$

Once the $H$ 's have been determined we can drop the $y$ dependence of $\Omega_{\text {ref }}^{\mathrm{S}}\left(\gamma_{1}+\gamma_{2}+2 \gamma_{3}\right)$ and set $\Omega_{\mathrm{ref}}^{\mathrm{S}}\left(\gamma_{\ell} ; y\right)=1$. This gives

$$
\begin{aligned}
Q\left(\gamma_{1}+\gamma_{2}+\right. & \left.2 \gamma_{3} ; y\right) \\
=\Omega_{\mathrm{ref}}^{\mathrm{S}}\left(\gamma_{1}+\right. & \left.\gamma_{2}+2 \gamma_{3}\right)+\left(y-y^{-1}\right)^{-3}\left(y+y^{-1}\right)^{-1}\left\{y^{-k+1}-y^{k-1}\right. \\
& \left.+\frac{1}{4}(k-1)\left(y+y^{-1}\right)^{2}\left(y-y^{-1}\right)+\frac{1}{4}(-1)^{k / 2}\left(y-y^{-1}\right)^{3}\right\} \quad \text { for } k \text { even } \\
=\Omega_{\mathrm{ref}}^{\mathrm{S}}\left(\gamma_{1}+\right. & \left.\gamma_{2}+2 \gamma_{3}\right)+\left(y-y^{-1}\right)^{-3}\left(y+y^{-1}\right)^{-1}\left\{y^{k-1}-y^{-k+1}\right. \\
& \left.-\frac{1}{2}(k-1)\left(y^{2}-y^{-2}\right)\right\} \text { for } k \text { odd }
\end{aligned}
$$

We note that both for $k$ even and odd the negative powers of $y$ in this expression are given by

$$
Q\left(\gamma_{1}+\gamma_{2}+2 \gamma_{3} ; y\right) \simeq(-1)^{k+1} y^{-k+5}\left(1-y^{2}\right)^{-3}\left(1+y^{2}\right)^{-1} .
$$

Let us now compare this prediction with an explicit computation of the cohomology of the Higgs branch. Since the node 3 carries an U(2) gauge group, the fields $\phi_{23, \alpha}$ and $\phi_{31, \alpha}$

\footnotetext{
${ }^{16}$ For this solution the locations $x_{3}$ and $x_{4}$ coincide and hence the same solution also appears in the permutation $\{1,2,4,3\}$. But following our prescription we count the solution only once.
} 
carry an extra $\mathrm{U}(2)$ index which we shall label by $s .{ }^{17}$ The D-term equations for the U(1) factors take the form

$$
\begin{aligned}
\phi_{12, \alpha}^{*} \phi_{12, \alpha}-\phi_{31, \alpha, s}^{*} \phi_{31, \alpha, s} & =c_{1}, \\
-\phi_{12, \alpha}^{*} \phi_{12, \alpha}+\phi_{23, \alpha, s}^{*} \phi_{23, \alpha, s} & =c_{2}, \\
-\phi_{23, \alpha, s}^{*} \phi_{23, \alpha, s}+\phi_{31, \alpha, s}^{*} \phi_{31, \alpha, s} & =2 c_{3},
\end{aligned}
$$

while the D-term equations for the $\mathrm{SU}(2)$ gauge group further require

$$
\phi_{23, \alpha, s}^{*} T_{s s^{\prime}}^{a} \phi_{23, \alpha, s^{\prime}}-\phi_{31, \alpha, s}^{*} T_{s s^{\prime}}^{a} \phi_{31, \alpha, s^{\prime}}=0,
$$

where $T^{a}$ for $1=1,2,3$ are the Lie algebra generators (Pauli matrices in this case). The superpotential is given by

$$
W=C_{\alpha \beta \gamma} \phi_{12, \alpha} \phi_{23, \beta, s} \phi_{31, \gamma, s}
$$

where $C_{\alpha \beta \gamma}$ are constants. If we ignore the last set of equations (6.15) then solutions to $(6.14)$ can be found by choosing:

$$
\phi_{31, \alpha, s}=0, \quad \phi_{12, \alpha}^{*} \phi_{12, \alpha}=c_{1}>0, \quad \phi_{23, \alpha . s}^{*} \phi_{23, \alpha, s}=c_{1}+c_{2}>0, \quad C_{\alpha \beta \gamma} \phi_{12, \alpha} \phi_{23, \beta, s}=0 .
$$

This describes the complete intersection of $2 c$ hypersurfaces of degree $(1,1)$ inside $\mathbb{P}^{a-1} \times \mathbb{P}^{2 b-1}$, generating a manifold of complex dimension $a+2 b-2 c-2=k-2$. At generic points on this space, the $\mathrm{SU}(2)$ gauge symmetry is completely broken. The space of solutions to the $\mathrm{SU}(2)$ D-term equations (6.15) modulo the action of the compact gauge group is isomorphic to the quotient of the semi-stable locus by the complexified gauge group $\operatorname{SL}(2, \mathbb{C})$, and is a complex manifold $\mathcal{M}$ of dimension $k-5$. This agrees with the maximal negative power of $y$ in (6.13). Our goal is to compute the cohomology of this manifold $\mathcal{M}$ and compare it with (6.13).

For this purpose, we shall first consider the cohomology of the vacuum moduli space $\mathcal{M}_{0}$ in the absence of superpotential, i.e. the space of solutions to the $\mathrm{U}(1)$ and $\mathrm{SU}(2)$ D-term constraints (6.14) and (6.15) modulo the gauge group $\mathrm{U}(1) \times \mathrm{U}(1) \times \mathrm{U}(2)$. To compute the cohomology of $\mathcal{M}_{0}$, we shall use the HN recursion method described in section 2.5. ${ }^{18}$ Under the same assumptions as in (6.1), (6.2), we find that the slopes are ordered according to

$$
\gamma_{2}<\gamma_{2}+\gamma_{3}<\gamma_{2}+2 \gamma_{3}<2 \gamma_{3}<\gamma_{3}<\gamma_{1}+\gamma_{2}+\gamma_{3}<\gamma_{1}+\gamma_{2}<\gamma_{1}+2 \gamma_{3}<\gamma_{1}+\gamma_{3}<\gamma_{1} .
$$

Using (2.38), (2.39) we arrive at

$$
\begin{array}{rlrl}
\mathcal{I}\left(\gamma_{1}+\gamma_{2} ; w\right) & =\left(w^{a}-w^{-a}\right)\left(w-w^{-1}\right)^{-2}, & \mathcal{I}\left(\gamma_{2}+\gamma_{3} ; w\right)=0, & \mathcal{I}\left(\gamma_{1}+\gamma_{3} ; w\right)=0, \\
\mathcal{I}\left(2 \gamma_{3} ; w\right) & =w^{-1}\left(w-w^{-1}\right)^{-1}\left(w^{2}-w^{-2}\right)^{-1}, & \mathcal{I}\left(\gamma_{1}+2 \gamma_{3} ; w\right)=0, & \mathcal{I}\left(\gamma_{2}+2 \gamma_{3} ; w\right)=0, \\
\mathcal{I}\left(\gamma_{1}+\gamma_{2}+\gamma_{3} ; w\right) & =w^{a+b+c}\left(w-w^{-1}\right)^{-3}\left(w^{a}-w^{-a}\right)\left(w^{b}-w^{-b}\right) &
\end{array}
$$

\footnotetext{
${ }^{17}$ Even though we use the same symbol $s$ it should be understood that for $\phi_{23}$ it labels the antifundamental representation of $\mathrm{SU}(2)$ while for $\phi_{31}$ it labels the fundamental representation of $\mathrm{SU}(2)$.

${ }^{18}$ Alternatively, one can use Maxwell-Boltzmann statistics to compute $Q_{0}(\gamma ; y)$ from a set of Abelian quivers [6].
} 
hence, for the total charge vector $\gamma=\gamma_{1}+\gamma_{2}+2 \gamma_{3}$,

$$
\begin{aligned}
\mathcal{I}(\gamma ; w) & =h(\gamma ; w)-\mathcal{F}\left(\gamma_{1}+\gamma_{2}, 2 \gamma_{3} ; w\right)-\mathcal{F}\left(\gamma_{1}+\gamma_{2}+\gamma_{3}, \gamma_{3} ; w\right)-\mathcal{F}\left(\gamma_{1}, 2 \gamma_{3}, \gamma_{2} ; w\right) \\
& =w^{2 c}(w-1 / w)^{-4}(w+1 / w)^{-1}\left(w^{a}-w^{-a}\right)\left(w^{b}-w^{-b}\right)\left(w^{b-1}-w^{1-b}\right)
\end{aligned}
$$

and therefore

$$
Q_{0}(\gamma ; y)=(-1)^{a+1} y^{5-a-2 b-2 c}\left(1-y^{2}\right)^{-3}\left(1+y^{2}\right)^{-1}\left(1-y^{2 a}\right)\left(1-y^{2 b}\right)\left(1-y^{2 b-2}\right) .
$$

Thus, the Betti numbers of $\mathcal{M}_{0}$ are given by

$$
\sum_{p} b_{p}\left(\mathcal{M}_{0}\right)(-y)^{p}=\left(1-y^{2}\right)^{-3}\left(1+y^{2}\right)^{-1}\left(1-y^{2 a}\right)\left(1-y^{2 b}\right)\left(1-y^{2 b-2}\right) .
$$

The effect of the F-term constraints is to set $\phi_{31, \alpha, s}=0$ and impose the last set of equations in (6.17). Setting $\phi_{31, \alpha, s}$ to zero does not affect the Betti numbers, while by Lefschetz hyperplane theorem imposing the last set of equations of (6.17) does not change the Betti numbers for $p<k-5$. Thus the Laurent polynomial of the quiver moduli space is given by

$$
Q(\mathcal{M} ; y) \simeq(-1)^{k+1} y^{-k+5}\left(1-y^{2}\right)^{-3}\left(1+y^{2}\right)^{-1}\left(1-y^{2 a}\right)\left(1-y^{2 b}\right)\left(1-y^{2 b-2}\right) .
$$

Now it follows from the inequalities (6.1) that we can drop the $y^{2 a}, y^{2 b}$ and $y^{2 b-2}$ terms from (6.23) without affecting the negative powers of $y$. This gives

$$
Q(\mathcal{M} ; y) \simeq(-1)^{k+1} y^{-k+5}\left(1-y^{2}\right)^{-3}\left(1+y^{2}\right)^{-1}
$$

in perfect agreement with (6.13).

Before leaving this example we should draw the readers' attention to a subtle point. We could solve the U(1) D-term constraints (6.14) as well as the F-constraint coming from the superpotential (6.16) by choosing:

$$
\phi_{31, \alpha, s}=0, \quad \phi_{12, \alpha}^{*} \phi_{12, \alpha}=c_{1}, \quad \phi_{23, \alpha, s}=f_{\alpha} u_{s}, \quad u_{s}^{*} u_{s}=1, \quad f_{\alpha}^{*} f_{\alpha}=c_{1}+c_{2}, \quad C_{\alpha \beta \gamma} \phi_{12, \alpha} f_{\beta}=0 .
$$

This gives a codimension $c$ hypersurface in $\mathbb{P}^{a-1} \times \mathbb{P}^{b-1} \times \mathbb{P}^{1}$ spanned by $\phi_{12, \alpha}, f_{\alpha}$ and $u_{s}$ respectively and has dimension $(a+b-c-1)$. This seems to be larger that the dimension $(a+2 b-2 c-2)$ of the manifold we found earlier, since we have $b<c$. However, these solutions do not satisfy the $\mathrm{SU}(2)$ D-term constraint (6.15), as they would require $u_{s}^{*} T_{s s^{\prime}}^{a} u_{s^{\prime}}=0$. This will give $u_{s}=0$ and hence is inconsistent with the normalization of $u_{s}$ given in (6.25). Thus, the set of solutions (6.25) does not belong to the semi-stable locus.

\subsection{Rank $(1,1, N)$}

We now generalize the previous example to allow for a $\mathrm{U}(N)$ gauge group at the third node, keeping $\mathrm{U}(1)$ gauge groups at the first two nodes. We choose the FI parameters as in (6.2) and assume, for reasons to become apparent shortly, that the arrow multiplicities satisfy

$$
(c-b) N<a<(c-b) N+2 b, \quad b<c .
$$


In this case it is easy to see that

$$
\begin{gathered}
g_{\mathrm{ref}}\left(\gamma_{1}+\gamma_{2}+k_{0} \gamma_{3}, k_{1} \gamma_{3}, k_{2} \gamma_{3}, \cdots ; y\right)=0 \\
\Omega_{\mathrm{ref}}^{\mathrm{S}}\left(\gamma_{1}+p \gamma_{3}\right)=0, \quad \Omega_{\mathrm{ref}}^{\mathrm{S}}\left(\gamma_{2}+q \gamma_{3}\right)=0
\end{gathered}
$$

for positive integers $p, q, k_{1}, k_{2}, \cdots$ and non-negative integer $k_{0}$. Recalling that $\Omega_{\text {ref }}^{\mathrm{S}}\left(\gamma_{\ell} ; y\right)=1$ for $\ell=1,2,3$, we find that (1.4) takes the form

$$
\begin{aligned}
& Q\left(\gamma_{1}+\gamma_{2}+N \gamma_{3} ; y\right)=\Omega_{\mathrm{ref}}^{\mathrm{S}}\left(\gamma_{1}+\gamma_{2}+N \gamma_{3} ; y\right) \\
& +\sum_{\substack{s_{1}, s_{2}, \cdots \\
\sum m s_{m}=N}}\left(\prod_{m} \frac{1}{s_{m} !}\right) g_{\mathrm{ref}}\left(\gamma_{1}, \gamma_{2}, \gamma_{3}, \cdots \gamma_{3}, 2 \gamma_{3}, \cdots 2 \gamma_{3}, \cdots ; y\right) \prod_{m=1}^{\infty}\left(\frac{1}{m} \frac{y-y^{-1}}{y^{m}-y^{-m}}\right)^{s_{m}} \\
& +\sum_{\substack{k_{1}, k_{2}, \cdots \\
\sum_{i} k_{i}=N}} H\left(\left\{\gamma_{1}, \gamma_{2}, \gamma_{3}, \gamma_{3}, \cdots \gamma_{3}\right\},\left\{1,1, k_{1}, k_{2}, \cdots\right\} ; y\right) .
\end{aligned}
$$

In the second line $s_{1}$ represents the number of $\gamma_{3}$ 's, $s_{2}$ represents the number of $2 \gamma_{3}$ 's etc. in the argument of $g_{\text {ref }}$. Now this form is not suitable for determining the individual $H$ 's since we have set the $\Omega_{\text {ref }}^{\mathrm{S}}\left(\gamma_{3} ; y\right)=1$ from the beginning. These will be needed for analyzing bigger systems which include the current quiver as a subsystem. However for the purpose of finding $Q\left(\gamma_{1}+\gamma_{2}+N \gamma_{3} ; y\right)$ itself, we can proceed as follows. Since by construction the $H$ 's vanish as $y \rightarrow \infty$ and $y \rightarrow 0$, we see that they do not contribute negative powers of $y$ or constant term in a Laurent series expansion of (6.28) around $y=0$. Thus we have

$$
\begin{aligned}
& Q\left(\gamma_{1}+\gamma_{2}+N \gamma_{3} ; y\right) \simeq \\
& \quad \simeq \sum_{\substack{s_{1}, s_{2}, \cdots \\
\sum m s_{m}=N}}\left(\prod_{m} \frac{1}{s_{m} !}\right) g_{\mathrm{ref}}\left(\gamma_{1}, \gamma_{2}, \gamma_{3}, \cdots \gamma_{3}, 2 \gamma_{3}, \cdots 2 \gamma_{3}, \cdots ; y\right) \prod_{m=1}^{\infty}\left(\frac{1}{m} \frac{y-y^{-1}}{y^{m}-y^{-m}}\right)^{s_{m}},
\end{aligned}
$$

where $\simeq$ denotes equality of negative powers of $y$. The positive powers of $y$ in the Laurent polynomial $Q\left(\gamma_{1}+\gamma_{2}+N \gamma_{3} ; y\right)$ are then found using the $y \rightarrow 1 / y$ symmetry, and the constant term is given by $\Omega_{\text {ref }}^{\mathrm{S}}\left(\gamma_{1}+\gamma_{2}+N \gamma_{3}\right)$.

To proceed further we need to compute the Coulomb index $g_{\text {ref }}\left(\gamma_{1}, \gamma_{2}, \gamma_{3}, \cdots, 2 \gamma_{3}, \cdots ; y\right)$. For this we can proceed as in (6.5). Since the centers with charge $k_{i} \gamma_{3}$ do not interact among themselves, they must sit at one of the two possible locations $x_{\mathrm{m}}$ and $x_{\mathrm{r}}$ in (6.7). If we assume that the inequalities (6.26) are satisfied, then the analog of (6.8) shows that all the centers carrying charge proportional to $\gamma_{3}$ must in fact sit at $x_{\mathrm{r}}$, 1.e. the centers are arranged in the order $\left\{\gamma_{1}, \gamma_{2}, k_{1} \gamma_{3}, k_{2} \gamma_{3}, \cdots\right\}$ with all the $k_{i} \gamma_{3}$ 's coincident, and its reverse. This gives

$$
\begin{aligned}
g_{\mathrm{ref}}\left(\gamma_{1}, \gamma_{2}, \gamma_{3}, \cdots \gamma_{3}, 2 \gamma_{3}, \cdots 2 \gamma_{3}, \cdots ; y\right) \\
=(-1)^{k+\sum_{m} s_{m}-1}\left(y-y^{-1}\right)^{-\sum_{m} s_{m}-1}\left(y^{k}-(-1)^{\sum_{m} s_{m}} y^{-k}\right)
\end{aligned}
$$

where as before $s_{m}$ denotes the number of $m \gamma_{3}$ 's in the argument of $g_{\text {ref }}$, and

$$
k \equiv a+N(b-c)
$$


We now note that in a series expansion of (6.29) around $y=0$, the contribution from the first term inside the parantheses in (6.30), $y^{k}$, produces only positive powers of $y$. Thus we can drop this term for the purpose of computing the negative powers of $y$ in (6.29). This gives

$$
Q\left(\gamma_{1}+\gamma_{2}+N \gamma_{3} ; y\right) \simeq(-1)^{k+1} y^{-k+1}\left(1-y^{2}\right)^{-1} Q^{\prime}(N ; y)
$$

where

$$
Q^{\prime}(N ; y)=\sum_{\substack{s_{1}, s_{2}, \ldots \\ \sum_{m} m s_{m}=N}} \prod_{m=1}^{\infty}\left\{\frac{1}{s_{m} !}\left(\frac{1}{m} \frac{1}{y^{m}-y^{-m}}\right)^{s_{m}}\right\}
$$

Introducing the generating function ${ }^{19}$

$$
F(z ; y) \equiv \sum_{N=0}^{\infty} z^{N} Q^{\prime}(N ; y)
$$

and using (6.33), we find that $F(z ; y)$ is given by the $q$-deformed Pochhammer symbol,

$$
\begin{aligned}
F(z ; y) & =\exp \left[\sum_{m=1}^{\infty} z^{m} \frac{1}{m} \frac{1}{y^{m}-y^{-m}}\right]=\exp \left[-\sum_{m=1}^{\infty} \frac{1}{m} z^{m} y^{m} \sum_{n=0}^{\infty} y^{2 m n}\right] \\
& =\exp \left[\sum_{n=0}^{\infty} \ln \left(1-z y^{2 n+1}\right)\right]=\prod_{n=1}^{\infty}\left(1-z y^{2 n+1}\right) .
\end{aligned}
$$

To find $Q^{\prime}(N ; y)$ we need to extract the coefficient of $z^{N}$ in (6.35). The coefficient of the $z^{N}$ term in the Pochhammer symbol is given by $(-1)^{N} y^{N^{2}} /\left\{\left(1-y^{2}\right)\left(1-y^{4}\right) \cdots\left(1-y^{2 N}\right)\right\}$. Using (6.32) we now get

$$
Q\left(\gamma_{1}+\gamma_{2}+N \gamma_{3} ; y\right) \simeq(-1)^{k+N+1} y^{-k+N^{2}+1}\left(1-y^{2}\right)^{-2} \prod_{n=2}^{N}\left(1-y^{2 n}\right)^{-1}
$$

The analysis of the Higgs branch proceeds as in section 6.1. We arrive at the same set of equations (6.14)-(6.17) with the only difference that the index $s$ carried by $\phi_{31}$ labels the fundamental representation of $\mathrm{U}(N)$ and the index $s$ carried by $\phi_{23}$ runs over the anti-fundamental representation of $\mathrm{U}(N)$. The analog of (6.17) now gives a complete intersection hypersurface of codimension $N c$ in $\mathbb{P}^{a-1} \times \mathbb{P}^{N b-1}$. Thus we have a complex manifold of dimension $a+N b-N c-2=k-2$. The $\mathrm{SU}(N)$ D-term constraints reduces this to $k-N^{2}-1$. This agrees with the maximum negative power of $y$ in (6.36), showing that the Coulomb branch formula correctly reproduces the dimension of the moduli space.

To find the effect of the $\mathrm{SU}(N)$ D-term constraints on the cohomology we need to first find the Betti numbers of the manifold $\mathcal{M}_{0}$ that satisfies the D-term constraints but not the F-term constraints, and then use the Lefschetz hyperplane theorem to argue that the Betti numbers $b_{p}$ of the full moduli space coincide with those of $\mathcal{M}_{0}$ for $p<k-N^{2}-1$. We expect that this can be done using the HN recursion relations but we have not done this. Instead, we observe that the last factor $\prod_{n=2}^{N}\left(1-y^{2 n}\right)^{-1}$ in (6.36) is the Poincaré polynomial of the universal classifying space of $\mathrm{SU}(N)$ [28], which is known to govern the contribution of the semi-stable locus in the formalism of [26].

\footnotetext{
${ }^{19}$ Note that since the expression for $g_{\text {ref }}$ used in (6.30) is valid only when (6.26) holds, we can use this generating function to compute $Q\left(\gamma_{1}+\gamma_{2}+N \gamma_{3} ; y\right)$ only in this range.
} 


\subsection{Rank $(1,2,2)$}

In this final example, we consider again the same 3-node quiver (3.1) but with a U(1) factor at node 1 and $\mathrm{U}(2)$ factors at nodes 2 and 3 . For definiteness we take

$$
\gamma_{12}=a=3 k, \quad \gamma_{23}=b=2 k, \quad \gamma_{31}=c=3 k, \quad c_{1}=2, \quad c_{2}=-2.9, \quad c_{3}=1.9,
$$

where $k$ is a positive integer. Besides (3.17), in this case we also have the relations

$$
\begin{gathered}
\Omega_{\text {ref }}^{\mathrm{S}}\left(\gamma_{1}+2 \gamma_{2}\right)=\Omega_{\text {ref }}^{\mathrm{S}}\left(\gamma_{1}+2 \gamma_{3}\right)=0, \\
\Omega_{\text {scaling }}\left(\gamma_{1}+2 \gamma_{2}\right)=\Omega_{\text {scaling }}\left(\gamma_{1}+2 \gamma_{3}\right)=0,
\end{gathered}
$$

since the corresponding subquivers do not have closed oriented loops. This leads to

$$
\begin{aligned}
& Q\left(\gamma_{1}+2 \gamma_{2}+2 \gamma_{3} ; y\right)=\Omega_{\mathrm{ref}}^{\mathrm{S}}\left(\gamma_{1}+2 \gamma_{2}+2 \gamma_{3} ; y\right)+\Omega_{\mathrm{scaling}}\left(\gamma_{1}+2 \gamma_{2}+2 \gamma_{3} ; y\right) \\
& \quad+g_{\mathrm{ref}}\left(\gamma_{1}+\gamma_{2}+\gamma_{3}, \gamma_{2}, \gamma_{3} ; y\right)\left[\Omega_{\mathrm{ref}}^{\mathrm{S}}\left(\gamma_{1}+\gamma_{2}+\gamma_{3} ; y\right)+\Omega_{\text {scaling }}\left(\gamma_{1}+\gamma_{2}+\gamma_{3} ; y\right)\right] \\
& \quad \times \Omega_{\mathrm{ref}}^{\mathrm{S}}\left(\gamma_{2} ; y\right) \Omega_{\mathrm{ref}}^{\mathrm{S}}\left(\gamma_{3} ; y\right) \\
& \quad+g_{\mathrm{ref}}\left(\gamma_{1}+2 \gamma_{2}+\gamma_{3}, \gamma_{3} ; y\right)\left[\Omega_{\mathrm{ref}}^{\mathrm{S}}\left(\gamma_{1}+2 \gamma_{2}+\gamma_{3} ; y\right)+\Omega_{\text {scaling }}\left(\gamma_{1}+2 \gamma_{2}+\gamma_{3} ; y\right)\right] \Omega_{\mathrm{ref}}^{\mathrm{S}}\left(\gamma_{3} ; y\right) \\
& \quad+g_{\mathrm{ref}}\left(\gamma_{1}+\gamma_{2}+2 \gamma_{3}, \gamma_{2} ; y\right)\left[\Omega_{\mathrm{ref}}^{\mathrm{S}}\left(\gamma_{1}+\gamma_{2}+2 \gamma_{3} ; y\right)+\Omega_{\mathrm{scaling}}\left(\gamma_{1}+\gamma_{2}+2 \gamma_{3} ; y\right)\right] \Omega_{\mathrm{ref}}^{\mathrm{S}}\left(\gamma_{2} ; y\right) \\
& \quad+\frac{1}{4(y+1 / y)} g_{\mathrm{ref}}\left(\gamma_{1}, \gamma_{2}, \gamma_{2}, 2 \gamma_{3} ; y\right) \Omega_{\mathrm{ref}}^{\mathrm{S}}\left(\gamma_{1} ; y\right) \Omega_{\mathrm{ref}}^{\mathrm{S}}\left(\gamma_{2} ; y\right)^{2} \Omega_{\mathrm{ref}}^{\mathrm{S}}\left(\gamma_{3} ; y^{2}\right) \\
& \quad+\frac{1}{4(y+1 / y)} g_{\mathrm{ref}}\left(\gamma_{1}, 2 \gamma_{2}, \gamma_{3}, \gamma_{3} ; y\right) \Omega_{\mathrm{ref}}^{\mathrm{S}}\left(\gamma_{1} ; y\right) \Omega_{\mathrm{ref}}^{\mathrm{S}}\left(\gamma_{2} ; y^{2}\right) \Omega_{\mathrm{ref}}^{\mathrm{S}}\left(\gamma_{3} ; y\right)^{2} \\
& \quad+\frac{1}{4(y+1 / y)^{2}} g_{\mathrm{ref}}\left(\gamma_{1}, 2 \gamma_{2}, 2 \gamma_{3} ; y\right) \Omega_{\mathrm{ref}}^{\mathrm{S}}\left(\gamma_{1} ; y\right) \Omega_{\mathrm{ref}}^{\mathrm{S}}\left(\gamma_{2} ; y^{2}\right) \Omega_{\mathrm{ref}}^{\mathrm{S}}\left(\gamma_{3} ; y^{2}\right) \\
& \quad+\frac{1}{4} g_{\mathrm{ref}}\left(\gamma_{1}, \gamma_{2}, \gamma_{2}, \gamma_{3}, \gamma_{3} ; y\right) \Omega_{\mathrm{ref}}^{\mathrm{S}}\left(\gamma_{1} ; y\right) \Omega_{\mathrm{ref}}^{\mathrm{S}}\left(\gamma_{2} ; y\right)^{2} \Omega_{\mathrm{ref}}^{\mathrm{S}}\left(\gamma_{3} ; y\right)^{2}
\end{aligned}
$$

In this case from $(2.5)$ we have

$$
g_{\mathrm{ref}}\left(\gamma_{1}+2 \gamma_{2}+\gamma_{3}, \gamma_{3} ; y\right)=0, \quad g_{\mathrm{ref}}\left(\gamma_{1}+\gamma_{2}+2 \gamma_{3}, \gamma_{2} ; y\right)=0
$$

Using $(6.40),(2.7)$ and that $\Omega_{\mathrm{ref}}^{\mathrm{S}}\left(2 \gamma_{2} ; y\right)=\Omega_{\mathrm{ref}}^{\mathrm{S}}\left(2 \gamma_{3} ; y\right)=0$, we can reduce (6.39) to

$$
\begin{aligned}
& Q \\
& \left.\quad \gamma_{1}+2 \gamma_{2}+2 \gamma_{3} ; y\right)=\Omega_{\mathrm{ref}}^{\mathrm{S}}\left(\gamma_{1}+2 \gamma_{2}+2 \gamma_{3} ; y\right) \\
& \quad+H\left(\left\{\gamma_{1}, \gamma_{2}, \gamma_{2}, \gamma_{3}, \gamma_{3}\right\},\{1,1,1,1,1\} ; y\right) \Omega_{\mathrm{ref}}^{\mathrm{S}}\left(\gamma_{1} ; y\right) \Omega_{\mathrm{ref}}^{\mathrm{S}}\left(\gamma_{2} ; y\right)^{2} \Omega_{\mathrm{ref}}^{\mathrm{S}}\left(\gamma_{3} ; y\right)^{2} \\
& \quad+H\left(\left\{\gamma_{1}, \gamma_{2}, \gamma_{3}, \gamma_{3}\right\},\{1,2,1,1\} ; y\right) \Omega_{\mathrm{ref}}^{\mathrm{S}}\left(\gamma_{1} ; y\right) \Omega_{\mathrm{ref}}^{\mathrm{S}}\left(\gamma_{2} ; y^{2}\right) \Omega_{\mathrm{ref}}^{\mathrm{S}}\left(\gamma_{3} ; y\right)^{2} \\
& \quad+H\left(\left\{\gamma_{1}, \gamma_{2}, \gamma_{2}, \gamma_{3}\right\},\{1,1,1,2\} ; y\right) \Omega_{\mathrm{ref}}^{\mathrm{S}}\left(\gamma_{1} ; y\right) \Omega_{\mathrm{ref}}^{\mathrm{S}}\left(\gamma_{2} ; y\right)^{2} \Omega_{\mathrm{ref}}^{\mathrm{S}}\left(\gamma_{3} ; y^{2}\right) \\
& \quad+H\left(\left\{\gamma_{1}, \gamma_{2}, \gamma_{3}\right\},\{1,2,2\} ; y\right) \Omega_{\mathrm{ref}}^{\mathrm{S}}\left(\gamma_{1} ; y\right) \Omega_{\mathrm{ref}}^{\mathrm{S}}\left(\gamma_{2} ; y^{2}\right) \Omega_{\mathrm{ref}}^{\mathrm{S}}\left(\gamma_{3} ; y^{2}\right) \\
& \quad+H\left(\left\{\gamma_{1}+\gamma_{2}+\gamma_{3}, \gamma_{2}, \gamma_{3}\right\},\{1,1,1\} ; y\right) \Omega_{\mathrm{ref}}^{\mathrm{S}}\left(\gamma_{1}+\gamma_{2}+\gamma_{3} ; y\right) \Omega_{\mathrm{ref}}^{\mathrm{S}}\left(\gamma_{2} ; y\right) \Omega_{\mathrm{ref}}^{\mathrm{S}}\left(\gamma_{3} ; y\right) \\
& \quad+g_{\mathrm{ref}}\left(\gamma_{1}+\gamma_{2}+\gamma_{3}, \gamma_{2}, \gamma_{3} ; y\right) \Omega_{\mathrm{ref}}^{\mathrm{S}}\left(\gamma_{1}+\gamma_{2}+\gamma_{3} ; y\right) \Omega_{\mathrm{ref}}^{\mathrm{S}}\left(\gamma_{2} ; y\right) \Omega_{\mathrm{ref}}^{\mathrm{S}}\left(\gamma_{3} ; y\right) \\
& \quad+g_{\mathrm{ref}}\left(\gamma_{1}+\gamma_{2}+\gamma_{3}, \gamma_{2}, \gamma_{3} ; y\right) H\left(\left\{\gamma_{1}, \gamma_{2}, \gamma_{3}\right\},\{1,1,1\} ; y\right) \Omega_{\mathrm{ref}}^{\mathrm{S}}\left(\gamma_{1} ; y\right) \Omega_{\mathrm{ref}}^{\mathrm{S}}\left(\gamma_{2} ; y\right)^{2} \Omega_{\mathrm{ref}}^{\mathrm{S}}\left(\gamma_{3} ; y\right)^{2} \\
& \quad+\frac{1}{4(y+1 / y)} g_{\mathrm{ref}}\left(\gamma_{1}, \gamma_{2}, \gamma_{2}, 2 \gamma_{3} ; y\right) \Omega_{\mathrm{ref}}^{\mathrm{S}}\left(\gamma_{1} ; y\right) \Omega_{\mathrm{ref}}^{\mathrm{S}}\left(\gamma_{2} ; y\right)^{2} \Omega_{\mathrm{ref}}^{\mathrm{S}}\left(\gamma_{3} ; y^{2}\right)
\end{aligned}
$$




$$
\begin{aligned}
& +\frac{1}{4(y+1 / y)} g_{\mathrm{ref}}\left(\gamma_{1}, 2 \gamma_{2}, \gamma_{3}, \gamma_{3} ; y\right) \Omega_{\mathrm{ref}}^{\mathrm{S}}\left(\gamma_{1} ; y\right) \Omega_{\mathrm{ref}}^{\mathrm{S}}\left(\gamma_{2} ; y^{2}\right) \Omega_{\mathrm{ref}}^{\mathrm{S}}\left(\gamma_{3} ; y\right)^{2} \\
& +\frac{1}{4(y+1 / y)^{2}} g_{\mathrm{ref}}\left(\gamma_{1}, 2 \gamma_{2}, 2 \gamma_{3} ; y\right) \Omega_{\mathrm{ref}}^{\mathrm{S}}\left(\gamma_{1} ; y\right) \Omega_{\mathrm{ref}}^{\mathrm{S}}\left(\gamma_{2} ; y^{2}\right) \Omega_{\mathrm{ref}}^{\mathrm{S}}\left(\gamma_{3} ; y^{2}\right) \\
& +\frac{1}{4} g_{\mathrm{ref}}\left(\gamma_{1}, \gamma_{2}, \gamma_{2}, \gamma_{3}, \gamma_{3} ; y\right) \Omega_{\mathrm{ref}}^{\mathrm{S}}\left(\gamma_{1} ; y\right) \Omega_{\mathrm{ref}}^{\mathrm{S}}\left(\gamma_{2} ; y\right)^{2} \Omega_{\mathrm{ref}}^{\mathrm{S}}\left(\gamma_{3} ; y\right)^{2}
\end{aligned}
$$

The coefficients $g_{\text {ref }}\left(\gamma_{1}+\gamma_{2}+\gamma_{3}, \gamma_{2}, \gamma_{3} ; y\right), \quad H\left(\left\{\gamma_{1}+\gamma_{2}+\gamma_{3}, \gamma_{2}, \gamma_{3}\right\},\{1,1,1\} ; y\right)$, $H\left(\left\{\gamma_{1}, \gamma_{2}, \gamma_{3}\right\},\{1,1,1\} ; y\right)$ and $g_{\mathrm{ref}}\left(\gamma_{1}, 2 \gamma_{2}, 2 \gamma_{3} ; y\right)$ can all be evaluated from the results of section 3 using the assignments $(a, b, c)=(k, k, 2 k)$ for the first two cases, $(3 k, 3 k, 2 k)$ in the third case and $(6 k, 6 k, 8 k)$ in the last case (note that some permutations of the nodes are necessary in order to satisfy (3.3)). Thus from (3.22), (3.24) and the analysis of section 3.1 we have

$$
\begin{aligned}
g_{\mathrm{ref}}\left(\gamma_{1}+\gamma_{2}+\gamma_{3}, \gamma_{2}, \gamma_{3} ; y\right) & =0, \\
H\left(\left\{\gamma_{1}+\gamma_{2}+\gamma_{3}, \gamma_{2}, \gamma_{3}\right\},\{1,1,1\} ; y\right) & =0 \\
H\left(\left\{\gamma_{1}, \gamma_{2}, \gamma_{3}\right\},\{1,1,1\} ; y\right) & =-2\left(y-y^{-1}\right)^{-2} \\
g_{\mathrm{ref}}\left(\gamma_{1}, 2 \gamma_{2}, 2 \gamma_{3} ; y\right) & =\left(y-y^{-1}\right)^{-2}\left(y^{4 k}+y^{-4 k}\right) .
\end{aligned}
$$

Note that for the first two cases we cannot directly apply (3.22), (3.24) since we have $2 k=k+k$ and the triangle inequality is saturated. Instead we use the analysis given at the end of section 3.1 which leads to the vanishing of $g_{\text {ref }}$ and hence also $H .{ }^{20}$ Finally a direct computation gives

$$
\begin{aligned}
g_{\mathrm{ref}}\left(\gamma_{1}, \gamma_{2}, \gamma_{2}, 2 \gamma_{3} ; y\right) & =-\left(y-y^{-1}\right)^{-3}\left(y^{4 k}-y^{-4 k}\right), \\
g_{\mathrm{ref}}\left(\gamma_{1}, 2 \gamma_{2}, \gamma_{3}, \gamma_{3} ; y\right) & =-\left(y-y^{-1}\right)^{-3}\left(y^{4 k}-y^{-4 k}\right), \\
g_{\mathrm{ref}}\left(\gamma_{1}, \gamma_{2}, \gamma_{2}, \gamma_{3}, \gamma_{3} ; y\right) & =\left(y-y^{-1}\right)^{-4}\left(y^{4 k}+y^{-4 k}\right) .
\end{aligned}
$$

with the contributions coming from the permutations $\{4,1,2,3 ;+\}$ and its reverse for the first term, $\{2,1,3,4 ;-\}$ and its reverse for the second term and $\{2,3,1,4,5 ;+\}$ and its reverse for the last term.

Requiring that the coefficients of $\Omega_{\mathrm{ref}}^{\mathrm{S}}\left(\gamma_{1} ; y\right) \Omega_{\mathrm{ref}}^{\mathrm{S}}\left(\gamma_{2} ; y\right)^{2} \Omega_{\mathrm{ref}}^{\mathrm{S}}\left(\gamma_{3} ; y\right)^{2}$, $\Omega_{\text {ref }}^{\mathrm{S}}\left(\gamma_{1} ; y\right) \Omega_{\text {ref }}^{\mathrm{S}}\left(\gamma_{2} ; y^{2}\right) \quad \Omega_{\text {ref }}^{\mathrm{S}}\left(\gamma_{3} ; y\right)^{2}, \quad \Omega_{\text {ref }}^{\mathrm{S}}\left(\gamma_{1} ; y\right) \Omega_{\text {ref }}^{\mathrm{S}}\left(\gamma_{2} ; y\right)^{2} \Omega_{\mathrm{ref}}^{\mathrm{S}}\left(\gamma_{3} ; y^{2}\right) \quad$ and $\Omega_{\text {ref }}^{\mathrm{S}}\left(\gamma_{1} ; y\right) \Omega_{\mathrm{ref}}^{\mathrm{S}}\left(\gamma_{2} ; y^{2}\right) \Omega_{\mathrm{ref}}^{\mathrm{S}}\left(\gamma_{3} ; y^{2}\right)$ are Laurent polynomials in $y$, we get

$$
\begin{aligned}
H\left(\left\{\gamma_{1}, \gamma_{2}, \gamma_{2}, \gamma_{3}, \gamma_{3}\right\},\{1,1,1,1,1\} ; y\right) & =\left(y-y^{-1}\right)^{-4}\left\{-k^{2}\left(y-y^{-1}\right)^{2}-\frac{1}{2}\right\} \\
H\left(\left\{\gamma_{1}, \gamma_{2}, \gamma_{3}, \gamma_{3}\right\},\{1,2,1,1\} ; y\right) & =\frac{k}{2}\left(y-y^{-1}\right)^{-2}, \\
H\left(\left\{\gamma_{1}, \gamma_{2}, \gamma_{2}, \gamma_{3}\right\},\{1,1,1,2\} ; y\right) & =\frac{k}{2}\left(y-y^{-1}\right)^{-2},
\end{aligned}
$$

\footnotetext{
${ }^{20}$ Alternatively we could deform $\gamma_{12}, \gamma_{23}$ and $\gamma_{31}$ slightly away from those given in (6.37) - e.g. by adding small even integers to them for large $k$ - so that for the triple $\left(\gamma_{1}+\gamma_{2}+\gamma_{3}, \gamma_{2}, \gamma_{3}\right)$ strict triangle inequality holds, and take the limit back to the original values of $\gamma_{12}, \gamma_{23}$ and $\gamma_{31}$ at the end of the calculation. In that case we could use (3.22), (3.24) for the triple $\left(\gamma_{1}+\gamma_{2}+\gamma_{3}, \gamma_{2}, \gamma_{3}\right)$. The final result is unaffected by this.
} 


$$
H\left(\left\{\gamma_{1}, \gamma_{2}, \gamma_{3}\right\},\{1,2,2\} ; y\right)=-\frac{1}{2}\left(y+y^{-1}\right)^{-2}\left(y-y^{-1}\right)^{-2}
$$

Once the $H$ 's have been determined we can set $\Omega\left(\gamma_{\ell} ; y\right)=1$, and get, from (6.41),

$$
\begin{aligned}
Q\left(\gamma_{1}+2 \gamma_{2}+2 \gamma_{3} ; y\right)=\Omega_{\mathrm{ref}}^{\mathrm{S}}\left(\gamma_{1}+2 \gamma_{2}+2 \gamma_{3}\right)+\left(y-y^{-1}\right)^{-4}\left(y+y^{-1}\right)^{-2} \\
\quad \times\left[-k^{2} y^{4}-k^{2} y^{-4}+2 k^{2}-2 k+y^{2-4 k}+y^{4 k-2}+k y^{4}+k y^{-4}-y^{2}-y^{-2}\right] .
\end{aligned}
$$

It is easy to verify that the term inside the square bracket has $\left(y-y^{-1}\right)^{4}\left(y+y^{-1}\right)^{2}$ as a factor and hence (6.45) describes a Laurent polynomial in $y$. The negative powers of $y$ in this expansion are given by

$$
Q\left(\gamma_{1}+2 \gamma_{2}+2 \gamma_{3} ; y\right) \simeq y^{-4 k+6}\left(1-y^{2}\right)^{-4}\left(1+y^{2}\right)^{-2} .
$$

Let us now compare this result with an explicit computation of the cohomology of the Higgs branch. In this case the nodes 2 and 3 carry U(2) gauge groups. As a result the fields $\phi_{12, \alpha}$ and $\phi_{31, \alpha}$ carry an extra U(2) index each, and $\phi_{23, \alpha}$ carries an extra pair of $\mathrm{U}(2)$ indices. The $\mathrm{U}(1)$ D-term equations take the form:

$$
\begin{aligned}
\phi_{12, \alpha, s}^{*} \phi_{12, \alpha, s}-\phi_{31, \alpha, s^{\prime}}^{*} \phi_{31, \alpha, s^{\prime}} & =2, \\
-\phi_{12, \alpha, s}^{*} \phi_{12, \alpha, s}+\phi_{23, \alpha, s, t}^{*} \phi_{23, \alpha, s, t} & =-5.8, \\
-\phi_{23, \alpha, s, t}^{*} \phi_{23, \alpha, s, t}+\phi_{31, \alpha, t}^{*} \phi_{31, \alpha, t} & =3.8
\end{aligned}
$$

while the $\mathrm{SU}(2) \times \mathrm{SU}(2)$ D-term equations require

$$
\begin{aligned}
\phi_{23, \alpha, s, t}^{*} T_{s s^{\prime}}^{a} \phi_{23, \alpha, s^{\prime}, t}-\phi_{12, \alpha, s}^{*} T_{s s^{\prime}}^{a} \phi_{12, \alpha, s^{\prime}} & =0 \\
\phi_{23, \alpha, s, t}^{*} T_{t t^{\prime}}^{a} \phi_{23, \alpha, s, t^{\prime}}-\phi_{31, \alpha, t}^{*} T_{t t^{\prime}}^{a} \phi_{31, \alpha, t^{\prime}} & =0 .
\end{aligned}
$$

The superpotential is given by

$$
W=C_{\alpha \beta \gamma} \phi_{12, \alpha, s} \phi_{23, \beta, s, t} \phi_{31, \gamma, t} .
$$

If we ignore (6.48) then a solution to (6.47) and (6.49) can be found by choosing:

$$
\phi_{23, \alpha, s, t}=0, \quad \phi_{12, \alpha, s}^{*} \phi_{12, \alpha, s}=6, \quad \phi_{31, \alpha, t}^{*} \phi_{31, \alpha, t}=4, \quad C_{\alpha \beta \gamma} \phi_{12, \alpha, s} \phi_{31, \gamma, t}=0 .
$$

This describes a complete intersection of $8 k$ hypersurfaces of degree $(1,1)$ in $\mathbb{P}^{6 k-1} \times \mathbb{P}^{6 k-1}$, hence is a manifold of complex dimension $4 k-2$. The $\mathrm{SU}(2) \times \mathrm{SU}(2)$ D-term constraints (6.48) together with the identification under gauge transformations lead to a manifold $\mathcal{M}$ of complex dimension $4 k-6$. This agrees with the maximal negative power of $y$ in (6.46). Our goal is to compute the cohomology of this manifold and compare it with (6.46).

For this purpose, we shall first consider the cohomology of the vacuum moduli space $\mathcal{M}_{0}$ in the absence of superpotential, i.e. the space of solutions to the D-term constraints (6.47) and (6.48) modulo the gauge group $\mathrm{U}(1) \times \mathrm{U}(2) \times \mathrm{U}(2)$. To compute the 
cohomology of $\mathcal{M}_{0}$, we shall use the $\mathrm{HN}$ recursion method described in section 2.5. Under the same assumptions (6.37), we find that the slopes are ordered according to

$$
\begin{aligned}
\gamma_{2} & <2 \gamma_{2}+\gamma_{3}<\gamma_{1}+2 \gamma_{2}<\gamma_{2}+\gamma_{3}<\gamma_{1}+2 \gamma_{2}+\gamma_{3}<\gamma_{1}+\gamma_{2}<\gamma_{1}+2 \gamma_{2}+2 \gamma_{3}<\gamma_{2}+2 \gamma_{3} \\
& <\gamma_{1}+\gamma_{2}+\gamma_{3}<\gamma_{1}+\gamma_{2}+2 \gamma_{3}<\gamma_{3}<\gamma_{1}+2 \gamma_{3}<\gamma_{1}+\gamma_{3}<\gamma_{1}
\end{aligned}
$$

Thus we find

$$
\begin{aligned}
\mathcal{I}\left(\gamma_{1}+\gamma_{3} ; w\right) & =\mathcal{I}\left(\gamma_{2}+\gamma_{3} ; w\right)=\mathcal{I}\left(\gamma_{1}+2 \gamma_{3} ; w\right)=\mathcal{I}\left(\gamma_{2}+2 \gamma_{3} ; w\right)=0, \\
\mathcal{I}\left(2 \gamma_{2}+\gamma_{3} ; w\right) & =\mathcal{I}\left(2 \gamma_{2}+2 \gamma_{3} ; w\right)=0, \\
\mathcal{I}\left(\gamma_{1}+\gamma_{2} ; w\right) & =\left(w^{a}-w^{-a}\right) /\left(w-w^{-1}\right)^{2}, \\
\mathcal{I}\left(\gamma_{1}+2 \gamma_{2} ; w\right) & =\left(w^{a}-w^{-a}\right)\left(w^{a-1}-w^{1-a}\right)\left(w-w^{-1}\right)^{-3}\left(w+w^{-1}\right)^{-1}, \\
\mathcal{I}\left(\gamma_{1}+\gamma_{2}+\gamma_{3} ; w\right) & =w^{b}\left(w^{a}-w^{-a}\right)\left(w^{c}-w^{-c}\right)\left(w-w^{-1}\right)^{-3}, \\
\mathcal{I}\left(\gamma_{1}+2 \gamma_{2}+\gamma_{3} ; w\right) & =w^{2 b}\left(w^{a}-w^{-a}\right)\left(w^{a-1}-w^{1-a}\right)\left(w^{c}-w^{-c}\right)\left(w-w^{-1}\right)^{-4}\left(w+w^{-1}\right)^{-1}, \\
\mathcal{I}\left(\gamma_{1}+\gamma_{2}+2 \gamma_{3} ; w\right) & =w^{2 b}\left(w^{a}-w^{-a}\right)\left(w^{c}-w^{-c}\right)\left(w^{c-1}-w^{1-c}\right)\left(w-w^{-1}\right)^{-4}\left(w+w^{-1}\right)^{-1},(6.52)
\end{aligned}
$$

and, for the total charge vector $\gamma=\gamma_{1}+2 \gamma_{2}+2 \gamma_{3}$,

$$
\begin{aligned}
\mathcal{I}(\gamma ; w)= & h(\gamma ; w)-\mathcal{F}\left(\gamma_{1}, 2 \gamma_{3}, 2 \gamma_{2} ; w\right)-\mathcal{F}\left(\gamma_{3}, \gamma_{1}+2 \gamma_{2}+\gamma_{3} ; w\right) \\
& -\mathcal{F}\left(\gamma_{3}, \gamma_{1}+\gamma_{2}+\gamma_{3}, \gamma_{2} ; w\right)-\mathcal{F}\left(2 \gamma_{3}, \gamma_{1}+2 \gamma_{2} ; w\right) \\
& -\mathcal{F}\left(2 \gamma_{3}, \gamma_{1}+\gamma_{2}, \gamma_{2} ; w\right)-\mathcal{F}\left(\gamma_{1}+\gamma_{2}+2 \gamma_{3}, \gamma_{2} ; w\right)
\end{aligned}
$$

finally arriving at

$$
Q_{0}(\gamma ; y)=y^{-4 b-2 a-2 c+8}\left(1-y^{2 a}\right)\left(1-y^{2 a-2}\right)\left(1-y^{2 c}\right)\left(1-y^{2 c-2}\right)\left(1-y^{2}\right)^{-4}\left(1+y^{2}\right)^{-2} .
$$

It follows that the Betti numbers of $\mathcal{M}_{0}$ are given by

$$
\sum_{p} b_{p}(-y)^{p}=\left(1-y^{2}\right)^{-4}\left(1+y^{2}\right)^{-2}\left(1-y^{2 a}\right)\left(1-y^{2 a-2}\right)\left(1-y^{2 c}\right)\left(1-y^{2 c-2}\right),
$$

where

$$
a=\gamma_{12}=3 k, \quad b=\gamma_{23}=2 k, \quad c=\gamma_{31}=3 k .
$$

The effect of the F-term constraints is to set $\phi_{23, \alpha, s, s^{\prime}}=0$ and impose the last set of equations in (6.50). Setting $\phi_{23, \alpha, s, s^{\prime}}$ to zero does not affect the Betti numbers since its effect is to restrict the $4 b$ free complex variables $\phi_{23, \alpha, s, s^{\prime}}$ to the origin, while by Lefschetz hyperplane theorem imposing the last set of equations of (6.50) does not change the Betti numbers for $p<4 k-6$. Thus

$$
\begin{aligned}
Q(\mathcal{M} ; y) & \simeq y^{-4 k+6}\left(1-y^{2}\right)^{-4}\left(1+y^{2}\right)^{-2}\left(1-y^{6 k}\right)\left(1-y^{6 k-2}\right)\left(1-y^{6 k}\right)\left(1-y^{6 k-2}\right) \\
& \simeq y^{-4 k+6}\left(1-y^{2}\right)^{-4}\left(1+y^{2}\right)^{-2} .
\end{aligned}
$$

This is in perfect agreement with (6.46), giving further evidence that the HN method works for quivers with loops and no superpotential. 


\section{Acknowledgments}

We are grateful to M. Berkooz, A. Collinucci and S. El-Showk for valuable discussions. J.M. thanks the Amsterdam Summer Workshop on String Theory for its hospitality during part of this work. B.P. and A.S. wish to express their gratitude to the ICTS in Bangalore for generous hospitality during part of this work. The work of A.S. was supported in part by the project 11-R\&D-HRI-5.02-0304 and the J. C. Bose fellowship of the Department of Science and Technology, India.

Open Access. This article is distributed under the terms of the Creative Commons Attribution License which permits any use, distribution and reproduction in any medium, provided the original author(s) and source are credited.

\section{References}

[1] F. Denef, Supergravity flows and D-brane stability, JHEP 08 (2000) 050 [hep-th/0005049] [INSPIRE].

[2] F. Denef, Quantum quivers and Hall/hole halos, JHEP 10 (2002) 023 [hep-th/0206072] [INSPIRE].

[3] B. Bates and F. Denef, Exact solutions for supersymmetric stationary black hole composites, JHEP 11 (2011) 127 [hep-th/0304094] [INSPIRE].

[4] F. Denef and G.W. Moore, Split states, entropy enigmas, holes and halos, JHEP 11 (2011) 129 [hep-th/0702146] [INSPIRE].

[5] M. Kontsevich and Y. Soibelman, Stability structures, motivic Donaldson-Thomas invariants and cluster transformations, arXiv:0811.2435 [INSPIRE].

[6] J. Manschot, B. Pioline and A. Sen, Wall crossing from Boltzmann black hole halos, JHEP 07 (2011) 059 [arXiv: 1011.1258] [INSPIRE].

[7] B. Pioline, Four ways across the wall, J. Phys. Conf. Ser. 346 (2012) 012017 [arXiv: 1103.0261] [INSPIRE].

[8] C. Bachas and E. Kiritsis, $F^{4}$ terms in $N=4$ string vacua, Nucl. Phys. Proc. Suppl. 55B (1997) 194 [hep-th/9611205] [INSPIRE].

[9] A. Gregori et al., $R^{2}$ corrections and nonperturbative dualities of $N=4$ string ground states, Nucl. Phys. B 510 (1998) 423 [hep-th/9708062] [INSPIRE].

[10] D. Gaiotto, G.W. Moore and A. Neitzke, Framed BPS states, arXiv:1006.0146 [INSPIRE].

[11] J. de Boer, S. El-Showk, I. Messamah and D. Van den Bleeken, Quantizing $N=2$ multicenter solutions, JHEP 05 (2009) 002 [arXiv:0807.4556] [INSPIRE].

[12] J. de Boer, S. El-Showk, I. Messamah and D. Van den Bleeken, A bound on the entropy of supergravity?, JHEP 02 (2010) 062 [arXiv:0906.0011] [INSPIRE].

[13] S. Lee and P. Yi, Framed BPS states, moduli dynamics and wall-crossing, JHEP 04 (2011) 098 [arXiv:1102.1729] [INSPIRE].

[14] H. Kim, J. Park, Z. Wang and P. Yi, Ab initio wall-crossing, JHEP 09 (2011) 079 [arXiv:1107.0723] [INSPIRE]. 
[15] J. Manschot, B. Pioline and A. Sen, A fixed point formula for the index of multi-centered $N=2$ black holes, JHEP 05 (2011) 057 [arXiv:1103.1887] [INSPIRE].

[16] A. Sen, Arithmetic of quantum entropy function, JHEP 08 (2009) 068 [arXiv:0903.1477] [INSPIRE].

[17] A. Sen, How do black holes predict the sign of the Fourier coefficients of Siegel modular forms?, Gen. Rel. Grav. 43 (2011) 2171 [arXiv: 1008.4209] [INSPIRE].

[18] A. Dabholkar, J. Gomes, S. Murthy and A. Sen, Supersymmetric index from black hole entropy, JHEP 04 (2011) 034 [arXiv:1009.3226] [INSPIRE].

[19] I. Bena, M. Berkooz, J. de Boer, S. El-Showk and D. Van den Bleeken, Scaling BPS solutions and pure-Higgs states, arXiv: 1205.5023 [INSPIRE].

[20] S.-J. Lee, Z.-L. Wang and P. Yi, Quiver invariants from intrinsic Higgs states, JHEP 07 (2012) 169 [arXiv: 1205.6511] [INSPIRE].

[21] A. Sen, Equivalence of three wall crossing formulae, arXiv:1112.2515 [INSPIRE].

[22] S.-J. Lee, Z.-L. Wang and P. Yi, BPS states, refined indices and quiver invariants, JHEP 10 (2012) 094 [arXiv: 1207.0821] [INSPIRE].

[23] F. Hirzebruch, Topological methods in algebraic geometry, Springer, (1978) revised edition, Germany (1995).

[24] E. Diaconescu and G.W. Moore, Crossing the wall: branes versus bundles, Adv. Theor. Math. Phys. 14 (2010) [arXiv:0706.3193] [INSPIRE].

[25] P. Griffiths and J. Harris, Principles of algebraic geometry, Wiley, U.S.A. (1994).

[26] F.C. Kirwan, Cohomology of quotients in symplectic and algebraic geometry, Princeton University Press, Princeton U.S.A. (1984).

[27] G. Harder and M.S. Narasimhan, On the cohomology groups of moduli spaces of vector bundles on curves, Math. Ann. 212 (1975) 215.

[28] M. Atiyah and R. Bott, The Yang-Mills equations over Riemann surfaces, Phil. Trans. Roy. Soc. Lond. A 308 (1983) 523 [INSPIRE].

[29] J. Manschot, BPS invariants of semi-stable sheaves on rational surfaces, arXiv:1109.4861 [INSPIRE].

[30] M. Reineke, The Harder-Narasimhan system in quantum groups and cohomology of quiver moduli, Invent. Math. 152 (2003) 349 [math.QA/0204059].

[31] J. Engel and M. Reineke, Smooth models of quiver moduli, Math. Z. 262 (2009) 817 [arXiv:0706.4306].

[32] D. Joyce, Configurations in Abelian categories. IV. Invariants and changing stability conditions, Adv. Math. 217 (2008) 125 [math.AG/0410268].

[33] D. Joyce and Y. Song, A theory of generalized Donaldson-Thomas invariants, Mem. Amer. Math. Soc. 217 (2012) [arXiv:0810.5645] [inSPIRE].

[34] D. Joyce, Generalized Donaldson-Thomas invariants, arXiv:0910.0105 [INSPIRE].

[35] S. Even and J. Gillis, Derangements and Laguerre polynomials, Math. Proc. Camb. Phil. Soc. 79 (1976) 135. 